\author{
TRABAJO FIN DE MÁSTER \\ MÁSTER EN QUÍMICA \\ UNIVERSIDAD DE GRANADA
}

Departamento de Química Analítica

\title{
EVALUACIÓN DE LA MICROEXTRACCIÓN LÍQUIDO- LÍQUIDO DISPERSIVA PARA LA DETERMINACIÓN DE PATULINA EN ZUMOS DE MANZANA MEDIANTE ELECTROFORESIS CAPILAR
}

María Dolores Víctor Ortega

Granada, 2011 


\section{Índice}

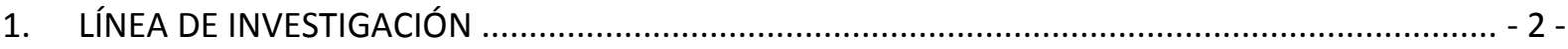

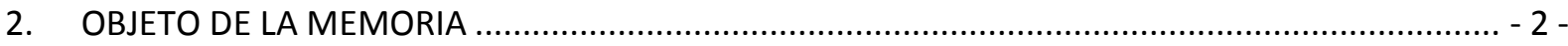

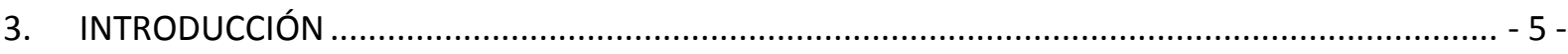

3.1. PATULINA

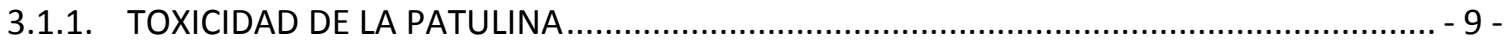

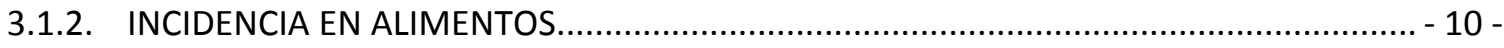

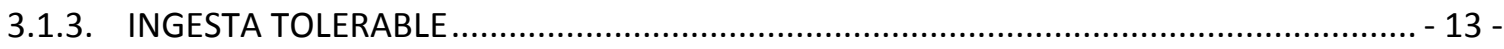

3.1.4. PRINCIPAL INTERFERENTE EN ZUMOS DE FRUTAS .................................................. - 14 -

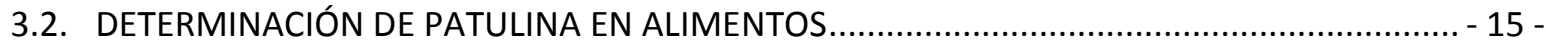

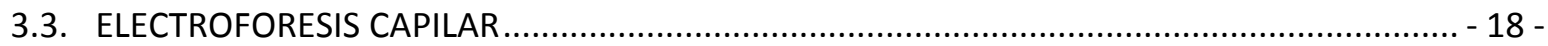

3.4. EXTRACCIÓN Y PURIFICACIÓN DE MICOTOXINAS …........................................................... 24 -

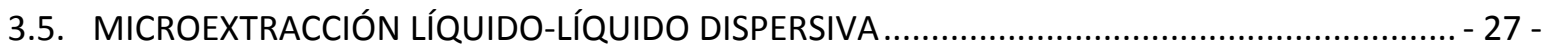

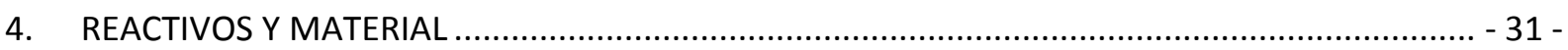

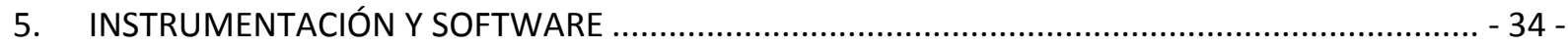

6. PROCEDIMIENTO ELECTROFORÉTICO PROPUESTO........................................................... - 36 -

7. PROCEDIMIENTO DE TRATAMIENTO DE MUESTRA MEDIANTE DLLME .................................. - 38 -

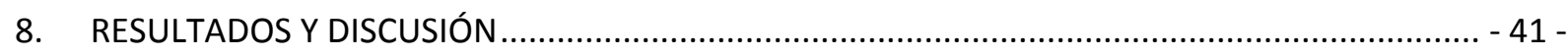

8.1. ESTUDIO DE LA SEPARACIÓN ELECTROFORÉTICA ........................................................ - 41 -

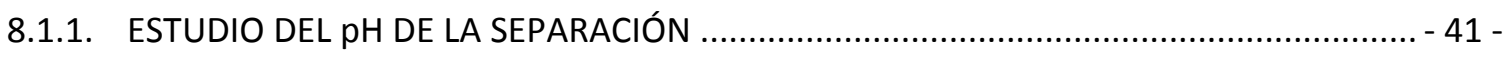

8.1.2. ESTUDIO DE LA NATURALEZA Y CONCENTRACIÓN DEL TAMPÓN ELECTROFORÉTICO - 44 -

8.1.3. ESTUDIO DE LA CONCENTRACIÓN DE SDS................................................................. - 46 -

8.1.4. ESTUDIO DEL VOLTAJE DE SEPARACIÓN ……............................................................. 47 -

8.1.5. ESTUDIO DE LA TEMPERATURA DEL CAPILAR ......................................................... 48 -

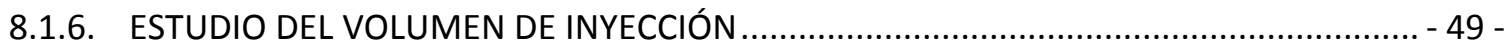

8.2. OPTIMIZACIÓN DE LA MICROEXTRACCIÓN LÍQUIDO-LÍQUIDO DISPERSIVA ......................... - 51 -

8.2.1. OPTIMIZACIÓN DE LA NATURALEZA DEL DISOLVENTE EXTRACTANTE......................... - 51 -

8.2.2. OPTIMIZACIÓN DE LA NATURALEZA DEL DISOLVENTE DISPERSIVO ............................. - 52 -

8.2.3. OPTIMIZACIÓN DEL VOLUMEN DE DISOLVENTE EXTRACTANTE …................................ - 53 -

8.2.4. OPTIMIZACIÓN DEL VOLUMEN DE DISOLVENTE DISPERSIVO ….................................... - 54 -

8.2.5. OPTIMIZACIÓN DEL PORCENTAJE DE SAL AÑADIDO …................................................. - 55 -

8.2.6. OPTIMIZACIÓN DEL VOLUMEN DE MUESTRA …....................................................... - 56 - 
8.2.7. OPTIMIZACIÓN DEL TIEMPO DE CENTRIFUGACIÓN $-57-$

8.3. ESTUDIOS PRELIMINARES EN ZUMOS DE FRUTA COMERCIALES $-58-$

8.4. CARACTERIZACIÓN DEL MÉTODO PROPUESTO. $-60-$

8.4.1. ESTABLECIMIENTO DE LA FUNCIÓN DE CALIBRADO, DE LOS PARÁMETROS ESTADÍSTICOS Y DE LOS LÍMITES DE DETECCIÓN Y CUANTIFICACIÓN. $-61-$

8.4.2. ESTUDIO DE LA PRECISIÓN $-62-$

8.4.3. ESTUDIO DE LA VERACIDAD DEL MÉTODO PROPUESTO $-62-$

9. VENTAJAS DE LA METODOLOGÍA PROPUESTA. $-65-$

10. APLICACIONES $-67-$

11. CONCLUSIONES $-69-$

12. GLOSARIO. $-71-$

13. REFERENCIAS 


\section{LÍNEA DE INVESTIGACIÓN}

\section{Y OBJETO DE LA MEMORIA}




\section{LÍNEA DE INVESTIGACIÓN}

El trabajo ha sido desarrollado dentro de la línea de investigación "DESARROLLO DE MÉTODOS PARA LA DETECCIÓN DE RESIDUOS DE CONTAMINANTES EN DIVERSOS TIPOS DE ALIMENTOS”, perteneciente al Grupo de Investigación “Química Analítica Alimentaria, Ambiental y Clínica (FQM-302)”, cuya responsable es la Dra. Ana $M^{\mathrm{a}}$ García Campaña y ha sido realizado bajo la dirección de las Dras. Ana $\mathrm{M}^{\mathrm{a}}$ García Campaña y Monsalud del Olmo Iruela.

\section{OBJETO DE LA MEMORIA}

La contaminación microbiológica de los alimentos es la causa principal de las enfermedades de origen alimentario. Dentro de este tipo de contaminación destacan las micotoxinas, sustancias producidas por ciertos hongos pertenecientes principalmente a los géneros Aspergillus, Fusarium y Penicillium. Estas sustancias suelen encontrarse en una gran variedad de productos agrícolas, almacenados en condiciones de humedad y aerobiosis, y son los contaminantes naturales de los alimentos más extendidos a nivel mundial. Se trata de productos altamente tóxicos, los cuales pueden producir mutaciones, cáncer, malformaciones en los fetos y disminuir la actividad del sistema inmune. Debido a su gran variedad de efectos tóxicos y, sobre todo, a su extrema resistencia al calor (termorresistencia), la presencia de las micotoxinas en los alimentos es considerada de alto riesgo para la salud humana y de los animales. La contaminación de los alimentos con micotoxinas es dependiente de las condiciones ambientales, que pueden propiciar el crecimiento del hongo y, por tanto, la producción de las toxinas. De este modo, la mayoría de los productos agrícolas pueden ser susceptibles de contaminación casi en cualquier momento, desde su producción en el campo, durante la cosecha, en el transporte y en el almacenamiento.

La patulina es una micotoxina producida por los hongos Penicillium griseofulvum ( $P$. patulum o $P$. urticae) en cereales y nueces, $P$. expansum en manzanas y en gran variedad de frutas, P. gladioli y P. sclerotigenum en rizomas y bulbos. Estos hongos contaminan principalmente en la etapa de postcosecha de la fruta, ya que la mayoría de las especies productoras de patulina crecen a temperaturas de refrigeración. La patulina, al igual que las demás micotoxinas, puede ser neurotóxica, inmunotóxica, inmunosupresiva, genotóxica, teratogénica y carcinogénica. Como resultado, la legislación a nivel europeo sobre seguridad 
alimentaria es cada vez más restrictiva en cuanto a los niveles de patulina en alimentos y ha establecido los contenidos máximos de esta micotoxina mediante el Reglamento (CE) $\mathrm{N}^{\circ}$ 1881/2006 [1]. Estos contenidos máximos se han establecido en $50 \mu \mathrm{g} \cdot \mathrm{kg}^{-1}$ para zumos de frutas, zumos de frutas concentrados reconstituidos y néctares de frutas, en $25 \mu \mathrm{g} \cdot \mathrm{kg}^{-1}$ para productos sólidos elaborados con manzanas, incluidos la compota y el puré de manzana destinados al consumo directo y, por último, en $10 \mu \mathrm{g} \cdot \mathrm{kg}^{-1}$ para zumo de manzana y productos sólidos elaborados a base de manzanas, incluidos la compota y el puré de manzana, destinados a los lactantes y niños de corta edad y para alimentos infantiles distintos de los alimentos elaborados a base de cereales lactantes y niños de corta edad.

Los métodos desarrollados para la determinación de patulina, habrán de presentar, por tanto, una adecuada selectividad, permitiendo efectuar análisis sin que interfieran los componentes de la matriz, así como una alta sensibilidad. De este modo, la etapa de tratamiento de muestra será, por tanto, decisiva en el diseño de tales métodos.

En los últimos años existe un creciente interés en la simplificación y miniaturización de los sistemas de tratamiento de muestra, introduciendo disolventes menos contaminantes y disminuyendo considerablemente los volúmenes utilizados, en línea con los principios de la llamada Química Verde o Química beneficiosa para el medio ambiente. Se trata de diseñar productos y procesos químicos que reduzcan o eliminen el uso y producción de sustancias peligrosas, implicando una mayor seguridad y un menor coste en relación a los procesos convencionales. Así, la microextracción líquido-líquido (liquid phase microextraction, LPME) es una técnica simple y económica en la que se requieren sólo unos microlitros de disolvente para concentrar a los analitos a partir de las muestras, en lugar de los elevados volúmenes requeridos en la extracción líquido-líquido convencional.

Este trabajo tiene como objeto principal la puesta a punto de una metodología de tratamiento de muestra LPME recientemente desarrollada denominada microextracción líquido-líquido dispersiva (dispersive liquid phase microextraction, DLLME), previa al análisis de patulina en zumos de manzana, que presenta la ventaja de ser eficaz, rápida y sencilla, además de reducir el consumo de disolventes orgánicos. Para ello, se hará uso de la cromatografía capilar electrocinética micelar (micellar electrokinetic capillary chromatography, MEKC) con detección UV-VIS, como técnica de separación, dada la naturaleza de la molécula y las ventajas que presenta esta metodología frente a los métodos habituales basados en cromatografía líquida. 


\section{INTRODUCCIÓN}




\section{INTRODUCCIÓN}

El reino de los hongos comprende a un grupo de organismos que se pueden clasificar en levaduras y hongos filamentosos o mohos. Estos últimos, que son los productores de micotoxinas, son organismos eucariotas multicelulares, constituidos por micelios verdaderos. Además carecen de clorofila y están formados por una serie de células alineadas, llamadas hifas. El micelio es el conjunto de hifas ramificadas, y resulta visible sobre el alimento donde se desarrolla, bien en superficie o en el interior, con un aspecto y color característicos. Los mohos utilizan para su crecimiento una serie de sustancias químicas denominadas metabolitos primarios, como pueden ser ácidos nucleicos, proteínas, carbohidratos y lípidos mayoritariamente. El uso de estos metabolitos primarios se asocia con una fase de rápido crecimiento (fase exponencial). Por su parte, los metabolitos secundarios son una serie de compuestos que no son esenciales para el crecimiento vegetativo en cultivo puro, y que incluyen a los antibióticos y a las micotoxinas.

Las micotoxinas, término derivado de las palabras griegas mikes y toxina, que significan hongo y veneno respectivamente, son metabolitos secundarios que se producen durante la etapa final de la fase de crecimiento del moho o al principio de la fase estacionaria (Figura 1), estando a menudo asociadas a la diferenciación y la esporulación. Los metabolitos secundarios se originan mayoritariamente en la ruta policetónica, siendo la cadena general policetónica del tipo R-CO-CH $2-\mathrm{CO}-\mathrm{CH}_{2}-\mathrm{CO}-$ $\mathrm{CH}_{2}-\mathrm{CO}-\mathrm{CH}_{2}-\mathrm{CO}-\mathrm{SCoA}$ de la cual se derivan la mayoría de las micotoxinas. Existen otras rutas biosintéticas pero son más complejas y esa complejidad se relaciona con un menor número de especies fúngicas capaces de elaborar micotoxinas [2]. 


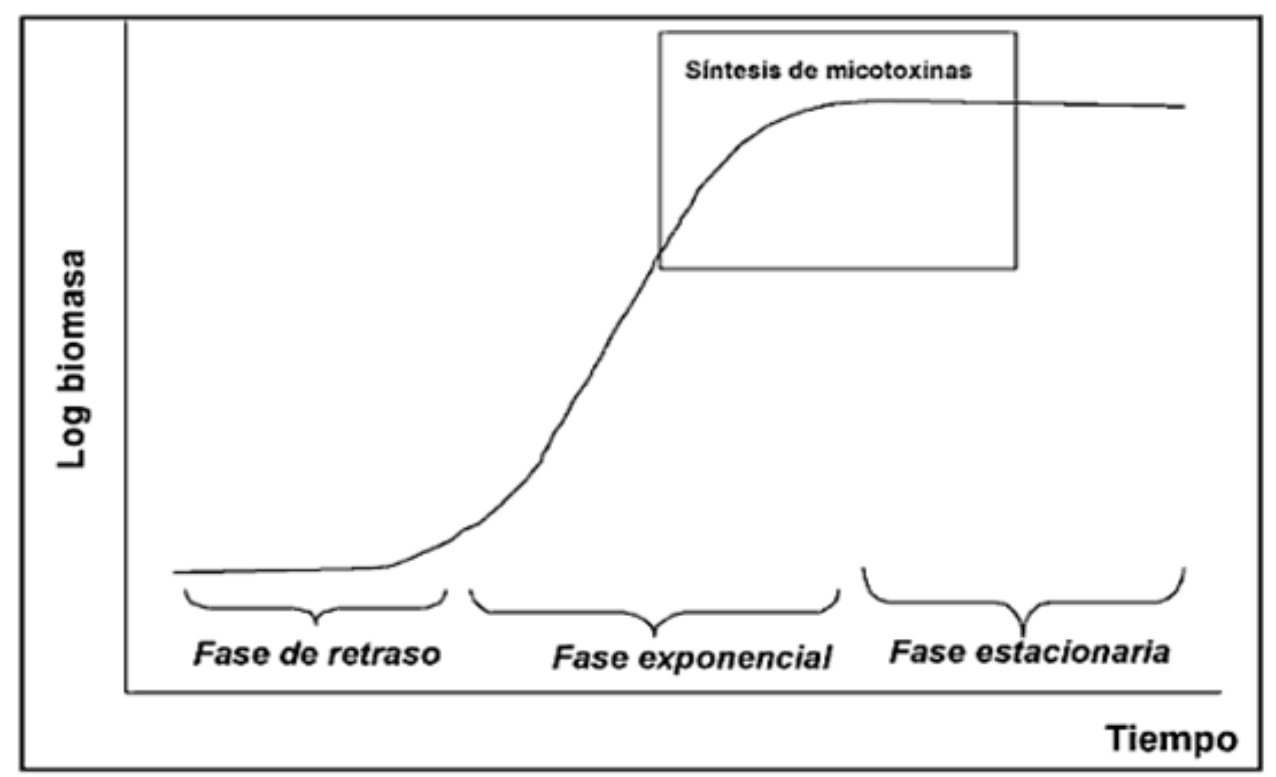

Figura 1. Fases de crecimiento fúngico y localización de la síntesis de micotoxinas

En cuanto a la toxicidad de las micotoxinas, éstas pueden contaminar alimentos, piensos, o las materias primas utilizadas para su elaboración, originando un grupo de enfermedades y trastornos, tanto en humanos como en animales, denominados micotoxicosis. La presencia de estas micotoxinas en los alimentos puede ser individual o simultánea con otras, lo que puede provocar efectos sinérgicos en su acción sobre el organismo, aumentando así su toxicidad.

Elevados niveles de micotoxinas en la dieta pueden causar efectos adversos agudos o crónicos sobre la salud del hombre y una gran variedad de especies animales. Los efectos adversos pueden afectar a distintos órganos, aparatos o sistemas, especialmente al hígado, riñón, sistema nervioso, endocrino e inmunitario. Los síntomas causados por las micotoxinas suelen ser tan diferentes unos de otros como lo son las propias estructuras químicas de dichas toxinas.

En términos generales, el riesgo de intoxicación aguda por micotoxinas en el hombre es bajo o moderado en comparación con intoxicaciones de origen microbiológico o por contaminantes químicos. No obstante, según algunos autores, en la exposición crónica y teniendo en cuenta la severidad de las lesiones que pueden causar, las micotoxinas presentan mayor riesgo tóxico que los contaminantes de origen antropogénico, aditivos alimentarios y plaguicidas, tal y como se muestra en la Figura 2. 


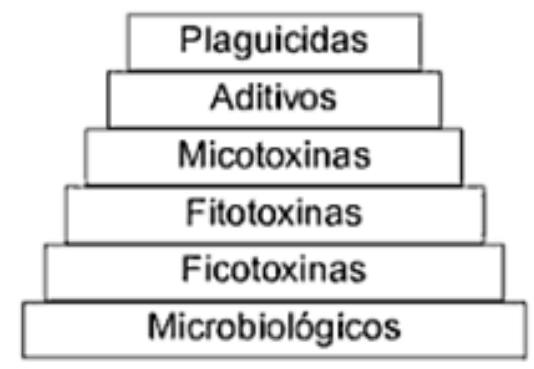

EFECTOS AGUDOS

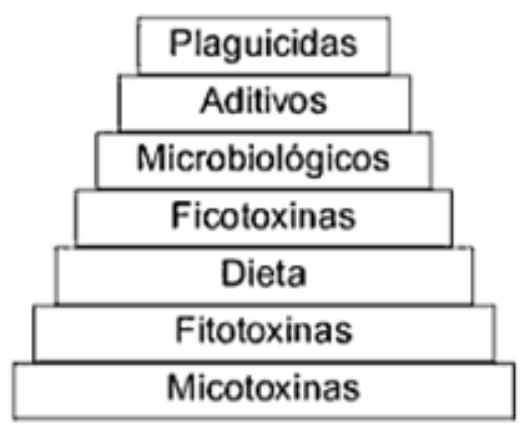

EFECTOS CRÓNICOS

Figura 2. Efectos sobre la salud humana de la exposición a distintos agentes [3]

Hay que destacar la posible interrelación entre las distintas micotoxinas consumidas conjuntamente que pueden presentar efecto sinérgico, aditivo, antagónico o de potenciación sobre la salud humana.

En cuanto a la toxicidad crónica, la Agencia Internacional de Investigación contra el Cáncer (IARC) clasifica varias micotoxinas como carcinógenas o potencialmente carcinógenas para el hombre, de acuerdo a los siguientes grupos:

Grupo 1: el agente es carcinógeno en humanos.

Grupo 2A: agente probablemente carcinógeno en humanos; existe limitada evidencia sobre humanos pero suficiente con animales.

- Grupo 2B: agente posiblemente carcinógeno; la evidencia en humanos es limitada y tampoco hay suficiente evidencia con animales en experimentación.

$\$$ Grupo 3: el agente no es clasificable como carcinógeno para humanos, pero tampoco hay evidencias de que no lo sea, y no puede incluirse en otro grupo.

- Grupo 4: el agente probablemente no es carcinógeno en humanos; la evidencia disponible, tanto de humanos como de experimentación animal así lo sugiere.

En la Tabla 1 se resume la evaluación realizada por la IARC en relación al poder carcinógeno de las micotoxinas. 
Tabla 1. Clasificación de las micotoxinas según la IARC [4]

\begin{tabular}{|l|c|}
\hline Micotoxinas & Grupo \\
\hline Aflatoxinas de origen natural & $2 \mathrm{~B}$ \\
\hline Aflatoxina $\mathrm{M}_{1}$ & 3 \\
\hline Citrina & $2 \mathrm{~B}$ \\
\hline Esterigmatocistina & $2 \mathrm{~B}$ \\
\hline Fumonisina $\mathrm{B}_{1}$ & $2 \mathrm{~B}$ \\
\hline Ocratoxina A & 3 \\
\hline Patulina & 3 \\
\hline $\begin{array}{l}\text { Toxinas derivadas de Fusarium graminearum, F. culmorum, F. } \\
\text { crookwellense (zearalenona, deoxinivalenol, nivaleno y }\end{array}$ & 3 \\
\hline fusarenona X) & \\
\hline Toxinas derivadas de Fusarium sporotrichioides (toxina T-2) & \\
\hline
\end{tabular}

\subsection{PATULINA}

La patulina es una micotoxina producida por una gran variedad de hongos. En la década de los 40, fue descrita por primera vez en Penicillium patulum, más tarde llamado Penicillium urticae y hoy conocido como Penicillium griseofulvum. Esta micotoxina es producida por $P$. griseofulvum en cereales y nueces, $P$. expansum en frutas, $P$. gladioli y P. sclerotigenum en rizomas y bulbos. También es producida por $P$. carneum, P. claverigerum, P. concentricum, P. coprobium, P. dipodomyicola, P. formosanum, P. glandicola, $P$. marinum, $P$. paneum y $P$. vulpinum. Otros organismos productores son: Paecilomyces variotii, Aspergillus clavatus, A. giganteus, A. terreus, Byssochlamys nivea y B. fulva. Estos hongos contaminan principalmente en la etapa de postcosecha de manzanas y peras, debido a que la mayoría de las especies productoras de patulina crecen a temperaturas de refrigeración.

La producción de patulina es dependiente de la temperatura y de la proporción de $\mathrm{CO}_{2}$ y oxígeno en el aire. Las condiciones óptimas de producción de patulina por $P$. expansum son: $\mathrm{pH}$ igual a $6 \mathrm{y}$ temperatura de $25^{\circ} \mathrm{C}$ en la pera y de $17^{\circ} \mathrm{C}$ en la manzana. Sin embargo, la patulina se puede formar entre 0 y $25^{\circ} \mathrm{C}$. Su producción es inhibida 
cuando se somete al hongo a una atmósfera que contenga $3 \%$ de $\mathrm{CO}_{2}$ y $2 \%$ de $\mathrm{O}_{2}$ a $25^{\circ} \mathrm{C}$ $[5]$.

La estructura química de la patulina, 4-hidroxi-4H-furo[3,2-c]piran-2(6H)-ona, se recoge en la Figura 3. Forma cristales incoloros con un punto de fusión de $111^{\circ} \mathrm{C} \mathrm{y}$ sublima en alto vacío a temperaturas de $70-100{ }^{\circ} \mathrm{C}$. La patulina es soluble en agua, metanol, etanol, acetona, acetato de etilo, éter y cloroformo, e insoluble en benceno y éter de petróleo. Su máximo de absorbancia se sitúa a $275 \mathrm{~nm}$ [6]. Es estable en medio ácido, pero puede descomponerse si se mantiene en ácido sulfúrico $2 \mathrm{~N}$ durante seis horas en ebullición; pierde su actividad biológica en medio alcalino ya que se hidroliza; se reduce en presencia de $\mathrm{SO}_{2}$ y en condiciones de fermentación. Al ser un alcohol secundario, reduce la solución de Fehling y decolora el permanganato de potasio.

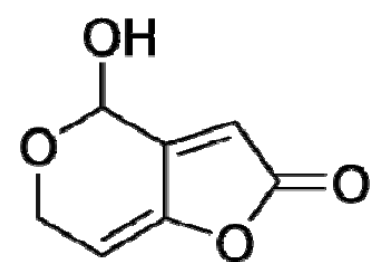

Figura 3. Estructura química de la patulina

\subsubsection{TOXICIDAD DE LA PATULINA}

Los efectos en la salud causados por la patulina están basados en un gran número de estudios hechos durante los últimos cincuenta años, los cuales implican efectos agudos, crónicos y a nivel celular. Los resultados de estos estudios son, en general, poco concluyentes pero sugieren que los síntomas por ingestión de patulina pueden ser agitación, convulsiones, congestión pulmonar, edema, ulceración, hiperemia, distensión del tracto gastrointestinal, hemorragia intestinal, degeneración de las células del epitelio, inflamación intestinal, vómito y otros daños a nivel gastrointestinal y de riñón.

El Comité de Mutagenicidad del Reino Unido ha clasificado a la patulina como mutagénica [7]. Un estudio realizado por el Comité Mixto FAO/OMS de Expertos en Aditivos Alimentarios (JECFA) concluye que esta micotoxina no tiene efectos teratogénicos en la reproducción, pero muestra embriotoxicidad acompañada de 
toxicidad materna. A dosis relativamente altas, la patulina tiene propiedades inmunotóxicas [8].

A nivel celular, se ha demostrado que la patulina tiene efectos que incluyen la disrupción de la membrana plasmática, inhibición de la síntesis de proteínas, inhibición del transporte de aminoácidos dependiente de sodio, interrumpe la traducción y la transcripción, inhibe la síntesis de $\mathrm{ADN}$ y al interferón que produce células $\gamma \mathrm{T}$ tipo 1 .

Sin embargo, como se ha indicado anteriormente, la IARC afirma que hoy día la patulina no es clasificable como carcinógeno para humanos.

\subsubsection{INCIDENCIA EN ALIMENTOS}

La presencia natural de la patulina en alimentos, principalmente en productos a base de manzanas, ha despertado cierta preocupación a nivel internacional. De acuerdo con estudios llevados a cabo por el Ministerio de Agricultura, Pesca y Alimentos de Inglaterra, entre 1980 y 1984, la incidencia de patulina en zumo de manzana era muy baja, de 38 a $56 \mu \mathrm{g} \cdot \mathrm{kg}^{-1}$. En aquel tiempo estos productos eran pasteurizados o envasados a altas temperaturas, pero en los últimos años la demanda de zumos naturales y $\sin$ aditivos alimentarios ha aumentado, lo que podría significar un aumento en la incidencia de esta micotoxina.

La Tabla 2 muestra la incidencia mundial de patulina en alimentos destinados al consumo humano: 
Tabla 2. Incidencia mundial de patulina en alimentos destinados al consumo humano [5]

\begin{tabular}{|c|c|c|c|}
\hline País & Alimento & Incidencia & $\begin{array}{c}\text { Valor máximo } \\
\left(\mu \mathrm{g} \cdot \mathrm{L}^{-1} 0 \mu \mathrm{g} \cdot \mathrm{kg}^{-1}\right)\end{array}$ \\
\hline \multirow{8}{*}{ Alemania } & Zumo de manzana & $27 / 70$ & 155 \\
\hline & Bebida de manzana & $11 / 64$ & 23 \\
\hline & Zumo de pera & $10 / 58$ & 25,4 \\
\hline & Zumo de uva & $2 / 61$ & 31,5 \\
\hline & Zumo de frutas & $1 / 57$ & 30 \\
\hline & Puré de tomate & $1 / 13$ & 3,5 \\
\hline & Alimento para bebé & $1 / 34$ & 68 \\
\hline & Zumo de manzana concentrado & $2.975 / 4.931$ & 3.533 \\
\hline \multirow[t]{2}{*}{ Australia } & Zumos de pera, manzana y frutales & $75 / 328$ & 1.130 \\
\hline & Zumo de manzana & $4 / 6$ & 32 \\
\hline \multirow[t]{3}{*}{ Austria } & Zumo de manzana concentrado & $3 / 9$ & 65 \\
\hline & Zumo de uva & $2 / 21$ & 22 \\
\hline & Mosto de uva & $2 / 6$ & 23,6 \\
\hline \multirow[t]{2}{*}{ Bélgica } & Zumo de manzana & $23 / 29$ & 50 \\
\hline & Zumo de uva & $1 / 5$ & 36 \\
\hline Brasil & Zumo de manzana & $1 / 111$ & 17 \\
\hline \multirow[t]{3}{*}{ España } & Zumo de manzana & $82 / 100$ & 170 \\
\hline & Zumo de manzana & $37 / 67$ & 130 \\
\hline & Puré de manzana & $5 / 17$ & 86 \\
\hline \multirow[t]{3}{*}{ Francia } & Sidra & $28 / 92$ & 101 \\
\hline & Bebida de sidra & $93 / 118$ & 1.604 \\
\hline & Alimento para bebé & $2 / 10$ & 58 \\
\hline Holanda & Productos de manzana & $1 / 63$ & $<\mathrm{LOD}$ \\
\hline \multirow[t]{3}{*}{ Irán } & Zumo de manzana & $29 / 42$ & 285,3 \\
\hline & Zumo de manzana concentrado & $18 / 23$ & 148,8 \\
\hline & & & (Continúa) \\
\hline
\end{tabular}


Tabla 2. Incidencia mundial de patulina en alimentos destinados al consumo humano [5]

\begin{tabular}{|c|c|c|c|}
\hline País & Alimento & Incidencia & $\begin{array}{c}\text { Valor máximo } \\
\left(\mu \mathrm{g} \cdot \mathrm{L}^{-1} \mathrm{o} \mu \mathrm{g} \cdot \mathrm{kg}^{-1}\right)\end{array}$ \\
\hline \multirow{9}{*}{ Italia } & Manzana con piel & $17 / 21$ & 1.166 \\
\hline & Manzana sin piel & $7 / 21$ & 93 \\
\hline & Zumo de manzana & $18 / 21$ & 1.150 \\
\hline & Puré de manzana & $2 / 4$ & 3,16 \\
\hline & Alimento para bebé & $13 / 14$ & 6,39 \\
\hline & Pera & $5 / 9$ & 720 \\
\hline & Melocotón & $1 / 7$ & 23,3 \\
\hline & Manzana deshidratada & $3 / 7$ & 320 \\
\hline & Productos de manzana & $11 / 44$ & 74,2 \\
\hline \multirow{7}{*}{ Noruega } & Zumo de manzana concentrado & $1 / 13$ & 12 \\
\hline & nacional & & \\
\hline & $\begin{array}{l}\text { Zumo de manzana concentrado } \\
\text { de importación }\end{array}$ & $11 / 21$ & 65,6 \\
\hline & $\begin{array}{l}\text { Zumo de manzana concentrado } \\
\text { para consumidor }\end{array}$ & $3 / 11$ & 21,5 \\
\hline & Zumo de manzana & $21 / 57$ & 23,9 \\
\hline & Zumo de frutas & $3 / 19$ & 32,4 \\
\hline & Néctar de manzana & $5 / 84$ & 16,2 \\
\hline \multirow[t]{2}{*}{ Portugal } & Zumo de manzana sin clarificar & $4 / 8$ & 25,2 \\
\hline & Zumo de pera sin clarificar & $2 / 8$ & 23,4 \\
\hline \multirow{3}{*}{ Reino Unido } & Zumo de manzana concentrado & $208 / 758$ & 49 \\
\hline & Zumo de manzana sin clarificar & $1 / 17$ & 118 \\
\hline & Zumo de manzana & $4 / 155$ & 434 \\
\hline Sudáfrica & Zumos y productos de varias frutas & $16 / 60$ & 45 \\
\hline Suecia & Zumo de manzana & $5 / 39$ & 25 \\
\hline Turquía & Zumo de manzana & $215 / 215$ & 376 \\
\hline
\end{tabular}

En las tablas anteriores se observa que en varios países se han encontrado muestras con contenidos de patulina muy elevados lo que podría ser un riesgo para la población a largo plazo. Hay que considerar que estos datos fueron recogidos entre el 
año 1994 y 2005, anteriores al Reglamento de 2006 que fija el contenido máximo de patulina en productos alimenticios [1]. Los niveles de patulina en zumo de manzana en las que se utilizaron fungicidas sintéticos en las etapas de pre y/o postcosecha, se sitúan entre 244-3.993 $\mu \mathrm{g} / \mathrm{L}$, mientras que en zumos derivados de manzanas de agricultura ecológica se han encontrado niveles de hasta $45.000 \mu \mathrm{g} / \mathrm{L}$ [5]. También se ha encontrado patulina en legumbres, cereales humedecidos y en pienso. En pan se han encontrado niveles de $20-300 \mu \mathrm{g} \cdot \mathrm{kg}^{-1}$ y en remolacha de $12-3.700 \mu \mathrm{g} \cdot \mathrm{kg}^{-1}$.

Por otro lado, hay que resaltar que la fermentación destruye a la patulina, de modo que productos como la sidra, a pesar de estar fabricados a partir de manzana, no presentan contaminación, a menos que a la sidra se le adicione zumo de manzana sin fermentar con el fin de estabilizar el grado alcohólico.

\subsubsection{INGESTA TOLERABLE}

En 1994 el Comité Científico de Alimentos de la Unión Europea (UE) expresó su acuerdo con las conclusiones a las que llegaron la JECFA y la IARC sobre la patulina [9]. Estas conclusiones se basan en la evaluación que llevó a cabo la JECFA en 1990 [10], en la que se estableció una Ingesta Semanal Tolerable Provisional (ISTP) de $7 \mu \mathrm{g} / \mathrm{kg}^{-1}$, basada en un Nivel Sin Efectos Adversos Observables (NOAEL) de 0,1 $\mathrm{mg} / \mathrm{kg}$ pc/día (siendo pc el peso corporal) de un estudio de toxicidad/carcinogenicidad a largo plazo efectuado en ratas. La conclusión de la IARC fue que no se podía llevar a cabo una evaluación de la carcinogenicidad de la patulina en humanos y que no había evidencia adecuada de este efecto en animales de experimentación.

La JECFA llevó a cabo una revisión en 1995 de los estudios realizados. Dado que la patulina no se acumula en el cuerpo y debido al patrón de consumo de los productos que la contiene, la ISTP se cambió a Ingesta Diaria Tolerable Máxima Provisional (IDTMP) y fue establecida en $43 \mu \mathrm{g} / \mathrm{kg}$ pc [11].

En 2001, se evaluó la ingesta de patulina por la población de los estados miembros de la UE. Los resultados obtenidos revelaron que la exposición media parece ser notablemente inferior al nivel de IDTMP. No obstante, si se consideran los grupos específicos de consumidores, en particular los niños de corta edad, y se tienen en cuenta 
los peores casos, la exposición a la patulina es más importante, aunque todavía por debajo de la IDTMP. Por otro lado, la Administración Federal de Drogas y Alimentos (FDA) consideró el consumo de zumo de manzana de todos los grupos consumidores y a los niños los dividió en dos categorías de acuerdo a su edad, los niños menores de un año y niños de 1-2 años de edad. Se consideraron estas edades ya que son el grupo, comparado con otros grupos de edad, donde se consumen mayores cantidades de zumo de manzana en relación a su peso corporal. Niños mayores de dos años (de 2-10 años de edad) no se incluyeron en un grupo separado, ya que la relación de consumo de zumo de manzana respecto al peso corporal disminuye notablemente después de los dos años de edad.

Es importante destacar que, aunque la variedad de alimentos donde se encuentra la patulina en altas concentraciones es reducida, la ingesta de estos alimentos es alta. Por ejemplo, en EEUU el consumo por persona de manzanas (sin contar subproductos) es de $8,4 \mathrm{~kg} / \mathrm{año}$, mientras que en promedio en los países europeos es de 20,8 kg/año aproximadamente. En este sentido, la UE ha elaborado el Reglamento No 1881/2006, 19 diciembre 2006, por el que se fija el contenido máximo de determinados contaminantes, incluyendo las micotoxinas, en los productos alimenticios [1].

En el caso de la patulina, estos contenidos máximos se han establecido en 50 $\mu \mathrm{g} \cdot \mathrm{kg}^{-1}$ para zumos de frutas, zumos de frutas concentrados reconstituidos y néctares de frutas, en $25 \mu \mathrm{g} \cdot \mathrm{kg}^{-1}$ para productos sólidos elaborados con manzanas, incluidos la compota y el puré de manzana destinados al consumo directo $\mathrm{y}$, por último, en 10 $\mu \mathrm{g} \cdot \mathrm{kg}^{-1}$ para zumo de manzana y productos sólidos elaborados a base de manzanas, incluidos la compota y el puré de manzana, destinados a los lactantes y niños de corta edad y para alimentos infantiles distintos de los alimentos elaborados a base de cereales lactantes y niños de corta edad.

\subsubsection{PRINCIPAL INTERFERENTE EN ZUMOS DE FRUTAS}

Durante el procesado y almacenamiento de alimentos que contienen carbohidratos tienen lugar modificaciones químicas. 
El 5-hidroximetilfurfural (HMF) es uno de los compuestos formados por la degradación de los productos azucarados, en particular por deshidratación de la fructosa, como ocurre en la fruta. Su aparición en los alimentos, junto otros compuestos furfurales y la patulina, se considera como un parámetro de calidad de los mismos, relacionado con la frescura y la calidad de algunos alimentos [12,13].

La estructura del 5-Hidroximetilfurural se muestra en la Figura 4.

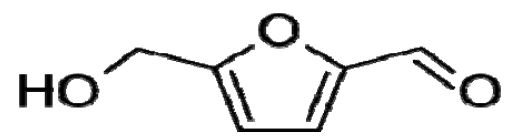

Figura 4. Estructura química del HMF

El Código Alimentario de la Organización Mundial de la Salud y la UE han establecido una concentración máxima de HMF en zumo de manzana de $50 \mathrm{mg} \cdot \mathrm{kg}^{-1}$, como indicador de descomposición y tratamiento a elevadas temperaturas. La Federación Internacional de Procesadores de Zumo de Fruta (con siglas en inglés IFFJP) recomienda una concentración máxima de 5-10 $\mathrm{mg} \cdot \mathrm{L}^{-1}$ de $\mathrm{HMF}$ en zumos de fruta y $25 \mathrm{mg} \cdot \mathrm{L}^{-1}$ en concentrados de fruta. Sin embargo, la relevancia toxicológica del HMF no está claramente demostrada aún [12].

El HMF es el principal interferente en los análisis de la patulina en muestras de zumos de frutas envasados, por lo que es necesario llevar a cabo una separación de ambos compuestos.

\subsection{DETERMINACIÓN DE PATULINA EN ALIMENTOS}

Se considera que alrededor del $20 \%$ de los alimentos de origen vegetal están contaminados por micotoxinas, por lo tanto, el análisis continuo de los alimentos en busca de estos contaminantes es importante, no solamente para la protección del consumidor sino también para los productores de alimentos. Esto implica el empleo de métodos de análisis contrastados que cumplan con unos parámetros de calidad establecidos y la verificación de los sistemas de control de seguridad alimentaria con el fin de preservar la salud de la población, desde la toma de muestra hasta el análisis del producto $[14,15]$. Por ello, la UE ha regulado los requerimientos mínimos que debe cumplir un método analítico para su aplicación en el control de estos contaminantes en 
alimentos [16]. Así, cuando la legislación comunitaria no exija ningún método específico para la determinación del contenido de micotoxinas en los productos alimenticios, los laboratorios podrán aplicar cualquier método de su elección, siempre que se ajuste a los criterios establecidos. En la Tabla 3 se recogen estos criterios para el caso concreto de la patulina.

Tabla 3. Criterios que deben cumplir los métodos de determinación de patulina en alimentos

\begin{tabular}{|c|c|c|c|}
\hline \multirow{2}{*}{$\begin{array}{c}\text { Concentración } \\
\left(\mu \mathrm{g} \cdot \mathrm{L}^{-1}\right)\end{array}$} & \multicolumn{3}{|c|}{ Patulina } \\
\hline & DER $_{\mathrm{r}} \%$ & $\operatorname{DER}_{\mathrm{R}} \%$ & Recuperación \% \\
\hline$<20$ & $\leq 30$ & $\leq 40$ & $50-120$ \\
\hline $20-50$ & $\leq 20$ & $\leq 30$ & $70-105$ \\
\hline$>50$ & $\leq 15$ & $\leq 25$ & $75-105$ \\
\hline \multicolumn{4}{|c|}{$\begin{array}{l}\text { DERr }=\text { Desviación estándar relativa, calculada a partir de los resultados generados bajo la } \\
\text { condiciones de repetibilidad. }\end{array}$} \\
\hline $\begin{array}{l}\mathrm{DER}_{\mathrm{R}}=\text { Desviación } \\
\text { de reproducibilidad. }\end{array}$ & ativa calcu & los resultac & os de las condicior \\
\hline
\end{tabular}

Además, existen diversos métodos de análisis recomendados para la determinación de micotoxinas en alimentos, como los recogidos por Association of Official Analytical Chemists (AOAC) [17], donde se pueden encontrar alrededor de cuarenta métodos validados para la determinación de diversas micotoxinas en gran variedad de alimentos, o los métodos normalizados propuestos por la Organización Internacional para la Normalización (International Standard Organization, ISO) y el Comité Europeo de Normalización (CEN) .

Los análisis requieren un alto grado de exactitud (precisión y veracidad), y por esta razón se recomienda el uso de métodos recomendados o la validación de los nuevos métodos propuestos a través de procedimientos que garanticen la calidad de los resultados, incluido el uso de materiales de referencia certificados, o de comparaciones interlaboratorio que ayudan a validar los métodos analíticos empleados y mejoran la competitividad entre laboratorios [18]. 
Algunos artículos de revisión recientes han recopilado los métodos propuestos para la determinación de micotoxinas en alimentos y sus principales aplicaciones [2126].

Centrándonos en los métodos de análisis para la determinación de patulina, la mayoría de ellos se han basado en la separación de los analitos por cromatografía en capa fina (TLC) [27], cromatografía de gases con espectrometría de masas (GC-MS) $[28,29]$ o cromatografía líquida [30]. La principal desventaja que presentan los métodos basados en TLC es el elevado límite de detección y en el caso de la cromatografía de gases, el hecho de que es necesario formar el correspondiente derivado volátil aunque también es posible confirmar patulina sin derivatizar mediante ionización química de ión negativo. También se ha llevado a cabo la confirmación tanto de patulina como de HMF en zumos mediante GC-MS tras trimetilsililación [31,32].

Sin embargo, el método más empleado para determinar patulina es la cromatografía líquida de alta resolución (HPLC) en fase inversa con detección ultravioleta/ visible (UV/Vis) ya que la patulina es un compuesto polar [33]. Considerando la coelución de la patulina con el 5-HMF, inicialmente se estableció un método de HPLC con detector de batería de diodos (DAD) para la determinación simultanea de ambos en zumo de manzana, usando una columna C18 y una mezcla agua:acetonitrilo como fase móvil [34]. Igualmente se demostró la necesidad de emplear pH ácido para prevenir la hidrólisis de la patulina [35]. Recientemente se ha utilizado elución en gradiente basada en una mezcla acidificada de agua-acetonitrilo-ácido perclórico $(0,01 \%$ ácido, v/v), ajustada a pH 3 como fase móvil para determinar los 3 furfuraldehídos: 5-HMF, 5-metilfurfural (5-MF) y 2-furfural (2-F), junto con la patulina en alimentos [12].

La principal ventaja de los métodos basados en HPLC-MS es la posibilidad de analizar la patulina sin derivatizar [36,37]. A pesar de la alta selectividad alcanzada por la MS con técnicas de ionización, tales como la ionización por electrospray (EI), la ionización química a presión atmosférica (APCI), o la fotoionización a presión atmosférica (APPI), estos instrumentos son caros para la determinación de este analito.

La CE ofrece muchas ventajas respecto de las técnicas cromatográficas convencionales, entre las que se incluyen una reducción importante del volumen de 
disolventes orgánicos utilizados, lo que implica una reducción de los residuos producidos; el pequeño volumen de muestra necesario y un aumento en la eficacia [38]. En cuanto al uso de CE para la determinación de patulina, se ha desarrollado un método basado en MEKC y detección UV/Vis a $276 \mathrm{~nm}$, utilizando tetraborato sódico como tampón electroforético a pH 9 y adicionando dodecil sulfato sódico (SDS) al mismo por encima de su concentración micelar critica (CMC) [39]. En algunos trabajos se adiciona además 5\% de acetonitrilo como modificador orgánico para la separación de la patulina y el HMF, su principal interferente $[40,41]$.

En esta Memoria se ha propuesto el uso de la CE como técnica separativa alternativa a las cromatográficas comúnmente empleadas en la determinación de patulina. A continuación, se muestran los fundamentos y aspectos más relevantes de esta técnica.

\subsection{ELECTROFORESIS CAPILAR}

Las separaciones mediante $\mathrm{CE}$ se basan en las diferencias entre las movilidades electroforéticas de los distintos analitos, como consecuencia de su diferente relación carga/tamaño, dentro de un tubo capilar lleno de disolución tampón cuando se somete a la acción de un campo eléctrico. Para llevar a cabo la separación son necesarios los siguientes elementos (Figura 5):

- Fuente de alto voltaje: capaz de aplicar hasta $30 \mathrm{kV}$.

- Capilar: generalmente son de sílice fundida y su diámetro interno oscila entre 25 y $100 \mu \mathrm{m}$ y el externo es de $375 \mu \mathrm{m}$.

- Detector: el más utilizado es el UV/Vis aunque también se pueden utilizar detectores de FL, MS, y menos frecuentemente, detectores de quimioluminiscencia (CL), fosforescentes y espectrometría Raman.

- Viales: además del vial de muestra son necesarios un vial con agua, otro con hidróxido sódico y otro con disolución tampón para acondicionar el capilar, un vial de desecho y dos más con tampón electroforético para cerrar el circuito cuando apliquemos el voltaje.

- Sistema de introducción de la muestra: el modo más habitual es aplicando presión sobre el vial de muestra (inyección hidrodinámica) 
aunque también se puede hacer mediante la aplicación de voltaje (inyección electrocinética). Otra posibilidad es elevando el vial de entrada para que la muestra entre por gravedad (inyección por efecto sifón).

- Sistema de termostatización del capilar: con este sistema se consigue evitar el aumento de la temperatura por efecto Joule, manteniéndola constante durante todo el análisis.

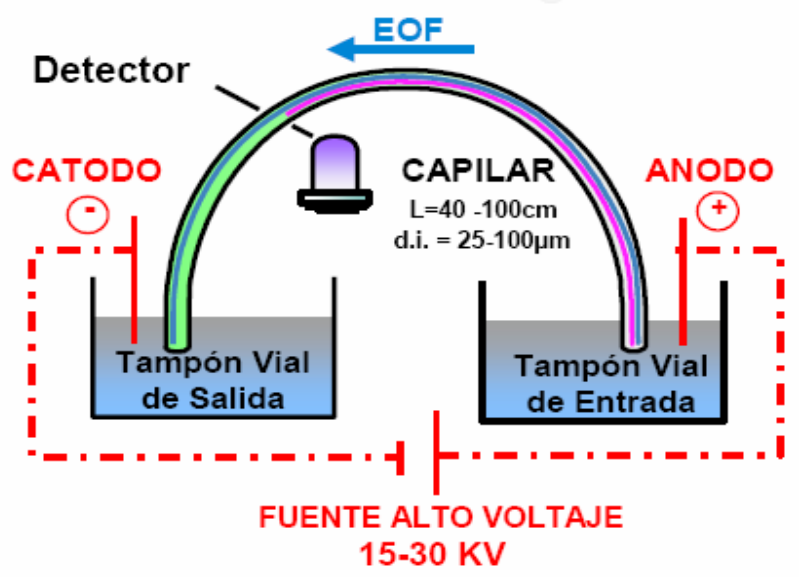

Figura 5. Sistema de electroforesis capilar

En las separaciones electroforéticas se dan dos fenómenos simultáneamente: la electroósmosis y la electromigración que dan lugar a dos flujos: el llamado flujo electroforético y el flujo electroosmótico (electroosmotic flow, EOF). El primero se produce por movimiento de los iones por acción del campo eléctrico aplicado y sólo afecta a las especies cargadas en disolución tampón que contrarrestan la carga negativa de los grupos silanoles que constituyen las paredes internas del capilar de sílice por aparición de una doble capa eléctrica. En CE, además de las moléculas de soluto cargadas, la disolución tampón también se desplaza por el capilar bajo la influencia del campo eléctrico, provocando la aparición del EOF, como consecuencia de que la superficie interna del capilar está cargada. Así, los cationes arrastran moléculas de disolvente en su movimiento hacia el cátodo lo que origina un flujo de disolvente en ese sentido, que aumenta cuanto mayor es el pH del tampón. El movimiento global de las especies en el medio electroforético en función de su relación carga/radio se representa en la Figura 6. 


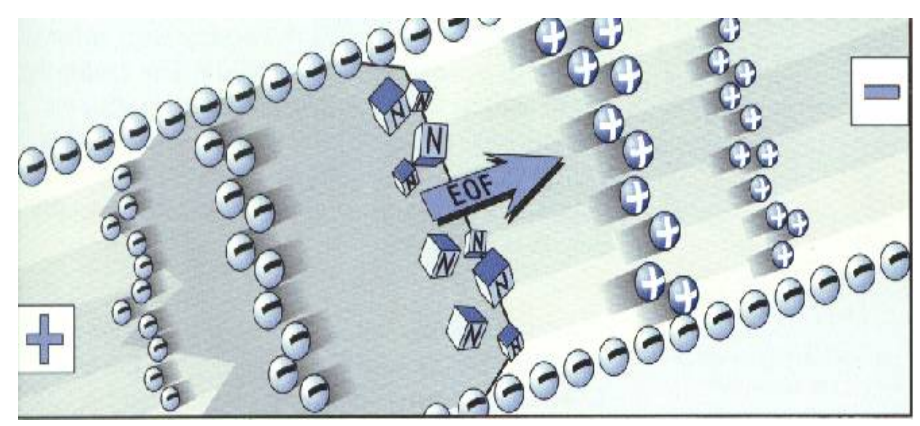

Figura 6. Migración diferencial de las moléculas según su carga/radio

El gran interés de la CE se debe, indudablemente, a la alta eficacia que presenta, con un mínimo consumo de disolventes y de muestras pero sobre todo, a la gran versatilidad en términos de modos de separación, los cuales, basándose en diferentes principios físicos-químicos, dan lugar a una amplia gama de selectividad en las determinaciones:

- $\quad$ Electroforesis capilar zonal (CZE): es la modalidad más empleada por ser la más sencilla. Consiste en rellenar todo el capilar con un electrolito de fondo que en la mayoría de los casos es un tampón.

- Electroforesis capilar en gel (CGE): el capilar se rellena con un gel. La separación se basa en las diferencias de tamaños entre las moléculas puesto que el gel actúa de criba.

- Electrocromatografía capilar (CEC): el capilar se rellena de fase estacionaria y los analitos se separan en base al equilibrio de partición entre la fase estacionaria y la fase móvil.

- Isoelectroenfoque capilar (CIEF): consiste en establecer un gradiente de pH a lo largo del capilar. Se ha empleado para separar proteínas y péptidos basándose en sus diferentes puntos isoeléctricos.

- Isotacoforesis capilar (CITP): es similar a CZE con la diferencia de que ahora el electrolito no es continuo, sino que el tampón de entrada no es el mismo que el de salida. Su limitación es que no podemos separar cationes y aniones a la vez.

- Cromatografía capilar electrocinética micelar (MEKC): éste ha sido el modo seleccionado en el trabajo realizado en la Tesis de Máster. Consiste en añadir un surfactante al tampón electroforético en una concentración 
superior a la concentración micelar crítica $(\mathrm{CMC})$, de modo que los compuestos neutros se distribuyen en un equilibrio entre dentro y fuera de las micelas formadas. Estas micelas, de forma esférica, tienen la parte hidrofílica de las moléculas de surfactante orientadas hacia la solución tampón, mientras que la parte hidrofóbica está orientada hacia el interior. Las moléculas neutras interaccionan con la micela en diferente grado en función de su hidrofobicidad, dando lugar a la separación (Figura 7). La micela actúa, de este modo, como fase pseudoestacionaria, ya que realiza una función similar a la de una fase cromatográfica. Los surfactantes más utilizados en MEKC son los aniónicos (como el SDS) y a pesar de su carga negativa, la micela o el conjunto micela-analito neutro migran hacia el cátodo arrastrados por el EOF. También han sido empleados surfactantes catiónicos, como el bromuro de deciltrimetilamonio (DTAB), no iónicos (Tritón X-100), zwitteriónicos, como el 3-[(3-Sulfonato de 3-[(3cholamidopropil)dimetilamonio]-1-propano (CHAPS) o sales biliares (ácido taurocólico). Este tipo de CE permite separar compuestos neutros y cargados a la vez. Si los solutos no son neutros, hay que tener en cuenta otros efectos: relación carga/masa, hidrofobicidad e interacción de carga. El uso de otros aditivos (acetonitrilo, metanol, ciclodextrinas, iones metálicos, etc.), además de las micelas, permite obtener mejoras en la selectividad y resolución.

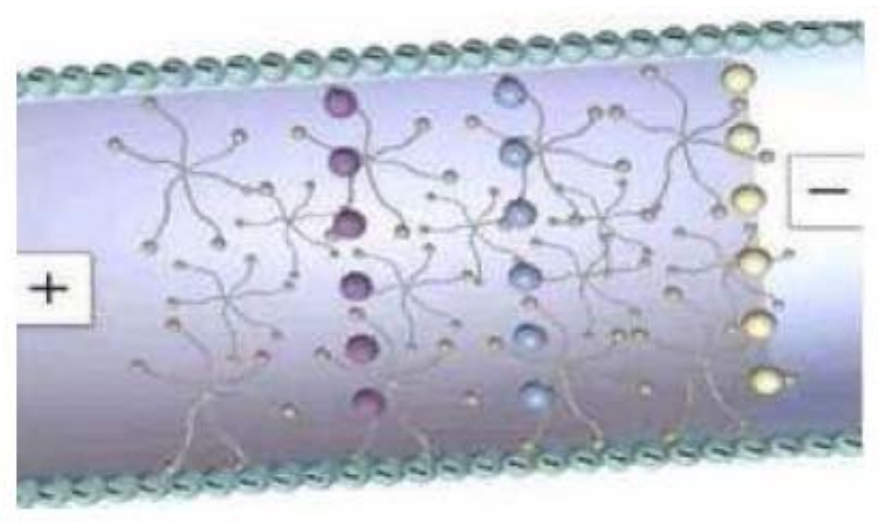

Figura 7. Esquema de separación en MEKC

Como resumen, las características y mecanismos de separación de estos modos de CE quedan reflejados en la Tabla 4. 
Tabla 4. Características y mecanismo de separación de los distintos modos de CE

\begin{tabular}{|c|c|c|}
\hline Modo & Características/aplicaciones & Mecanismo \\
\hline $\begin{array}{l}\text { Electroforesis capilar } \\
\text { zonal (CZE) }\end{array}$ & $\begin{array}{l}\text {-Capilar lleno de electrolitos de fondo, } \\
\text { aplicación de campo eléctrico. } \\
\text {-Aplicaciones: aminoácidos, péptidos, } \\
\text { proteínas, drogas, metabolitos, } \\
\text { contaminantes, iones inorgánicos, ácidos } \\
\text { orgánicos, compuestos quirales, fragmentos } \\
\text { de DNA, oligosacáridos... }\end{array}$ & $\begin{array}{l}\text { Separación de analitos } \\
\text { según su movilidad en } \\
\text { solución }\end{array}$ \\
\hline $\begin{array}{l}\text { Electroforesis capilar en } \\
\text { gel (CGE) }\end{array}$ & $\begin{array}{l}\text {-Capilar relleno con gel que funciona como } \\
\text { tamiz molecular. } \\
\text {-Aplicaciones: macromoléculas, ácidos } \\
\text { nucleicos y proteínas. }\end{array}$ & $\begin{array}{l}\text { Diferencia de tamaños y } \\
\text { cargas. }\end{array}$ \\
\hline $\begin{array}{l}\text { Electrocromatografía } \\
\text { capilar (CEC) }\end{array}$ & $\begin{array}{l}\text {-Capilar funcionalizado con fase estacionaria. } \\
\text {-Aplicaciones: macromoléculas, ácidos } \\
\text { nucleicos y proteínas. }\end{array}$ & Fenómenos de reparto \\
\hline $\begin{array}{l}\text { Isoelectroenfoque capilar } \\
\text { (CIEF) }\end{array}$ & $\begin{array}{l}\text {-Capilar lleno de electrolitos, cada uno capaz } \\
\text { de tamponar a un } \mathrm{pH} \text { diferente. Extremos del } \\
\text { capilar sumergidos en dos soluciones: una } \\
\text { ácida y otra básica. Se genera gradiente de } \\
\text { pHs en el capilar. } \\
\text {-Aplicaciones: sustancias anfotéricas como } \\
\text { péptidos, proteínas y aminoácidos. }\end{array}$ & $\begin{array}{l}\text { Punto isoeléctrico de los } \\
\text { analitos: migran hasta } \\
\text { alcanzar el pH donde son } \\
\text { neutros. }\end{array}$ \\
\hline $\begin{array}{l}\text { Isotacoforesis capilar } \\
\text { (CITP) }\end{array}$ & $\begin{array}{l}\text {-Sistema discontinuo de tampón: tampones } \\
\text { con distinta movilidad. Separación en zonas } \\
\text { consecutivas: escalones, no picos. } \\
\text {-Aplicaciones: iones metálicos, aniones } \\
\text { orgánicos e inorgánicos. }\end{array}$ & $\begin{array}{l}\text { Separación en el interior } \\
\text { de zona delimitada por } \\
\text { los dos tampones. }\end{array}$ \\
\hline $\begin{array}{l}\text { Cromatografía capilar } \\
\text { electrocinética micelar } \\
\text { (MEKC) }\end{array}$ & $\begin{array}{l}\text {-Capilar lleno de electrolito de fondo que } \\
\text { contiene un tensioactivo. } \\
\text {-Aplicaciones: aminoácidos, vitaminas, } \\
\text { fármacos e hidrocarburos aromáticos. }\end{array}$ & $\begin{array}{l}\text { Interacciones } \\
\text { hidrofóbicas/iónicas de } \\
\text { analitos con las micelas }\end{array}$ \\
\hline
\end{tabular}

La diferencia fundamental entre CE y HPLC reside en que la fuerza que hace avanzar a los analitos por la columna no es una bomba de alta presión sino el campo eléctrico aplicado, además de que en la separación en CE sólo se requieren capilares de 
sílice fundida y no columnas rellenas de fase estacionaria, lo cual conlleva múltiples ventajas como son:

- Empleo de volúmenes de muestra muy pequeños (1-40 nL), así como de reactivos, lo que la convierte en una técnica más barata y menos contaminante que la HPLC.

- Se trata también de una técnica rápida puesto que los tiempos de análisis suelen ser inferiores a 30 min., incluso a veces se consiguen separaciones en menos de 2 $\min$.

- $\mathrm{Su}$ característica más destacable es su alta eficacia, pudiéndose conseguir fácilmente cientos de miles de platos teóricos y en ocasiones hasta varios millones. La razón estriba fundamentalmente en su perfil de flujo plano en comparación con el perfil de flujo parabólico que presenta la HPLC.

- No se encuentra limitada por el peso molecular de los analitos, pudiendo separar, en la misma columna, desde pequeñas moléculas hasta otras mucho más complicadas.

El potencial analítico de la $\mathrm{CE}$ ha hecho que esta técnica de separación haya atraído desde sus comienzos a un número cada vez mayor de investigadores, lo que ha dado lugar a la publicación de numerosos libros y reviews que incluyen una buena descripción de la técnica y sus aplicaciones [42-47].

En lo que respecta a la determinación de micotoxinas, la CE no es una técnica que se haya usado frecuentemente, aunque algunos ejemplos de su empleo con detección $\mathrm{UV} / \mathrm{V}$ is son la determinación de ocratoxina A en vino $[48,49]$, moniliformina en maíz [50], o de patulina en sidra de manzana [39] y zumo [40,41]. 


\subsection{EXTRACCIÓN Y PURIFICACIÓN DE MICOTOXINAS}

Hasta ahora se han comentado las técnicas analíticas empleadas para la determinación de patulina. Sin embargo, previa a esta determinación es necesaria una etapa de preparación de la muestra para su análisis.

Las micotoxinas en los alimentos presentan una distribución poco uniforme por lo que se requiere una cuidadosa homogeneización de la muestra previa a la extracción de los residuos, que se suelen encontrar en concentraciones muy bajas. Por otra parte, la complejidad de los alimentos, donde se encuentran presentes cantidades importantes de proteínas, lípidos, hidratos de carbono, agua y otros componentes minoritarios de carácter nutricional o no, requiere, en no pocas ocasiones, una purificación para eliminar las sustancias interferentes, antes de proceder a la medida analítica.

Si la muestra es líquida se puede utilizar un disolvente inmiscible con ella. Sin embargo, existen varios inconvenientes para llevar a cabo este tipo de extracción, como es el elevado volumen de muestra y disolventes que se requieren, la baja selectividad junto a recuperaciones en ocasiones insuficientes y su dificultad de automatización. Por esta razón la extracción líquido-líquido no es muy empleada hoy en día. Cuando la muestra es de naturaleza sólida, los disolventes orgánicos más empleados para la extracción sólido-líquido son metanol, acetona, acetato de etilo, acetonitrilo, diclorometano, hexano y mezclas de ellos. La selección del disolvente se realiza dependiendo de la polaridad de la micotoxina y de la naturaleza de la muestra. La adición de sales y el ajuste del pH pueden contribuir a mejorar el rendimiento de las extracciones. Después de la extracción convencional es importante llevar a cabo una etapa de purificación o limpieza (clean-up) con objeto de eliminar posibles interferencias en la etapa de medida de la señal analítica.

Las técnicas de extracción y limpieza que han sido empleadas para la determinación de micotoxinas, junto con algunas referencias de sus aplicaciones en alimentos, son las siguientes:

Extracción en fase sólida:

- Extracción en fase sólida convencional (Solid Phase Extraction, SPE) [51-55]. 
- Extracción con columnas de intercambio iónico (Ion Exchange Column) [56].

- Extracción con columnas de inmunoafinidad (InmunoAffinity Column, IAC) [57].

- Microextracción en fase sólida (Solid Phase Micro-Extraction, SPME) [58].

- Dispersión de matriz en fase sólida (Matrix Solid Phase Dispersion, MSPD) [59,60].

Extracción líquido-líquido convencional (Liquid-Liquid Extraction, LLE) [61]

Extracción por fluidos supercríticos (Supercritical Fluid Extraction, SFE) $[62,63]$.

Extracción asistida por microondas (Microwave Assisted Extraction, MAE) [64].

Extracción acelerada por disolventes (Accelerated Solvent Extraction, ASE) $[65,66]$.

Un resumen de las principales ventajas e inconvenientes de estas técnicas de extracción de micotoxinas se muestra en la Tabla 5.

Tabla 5. Ventajas e inconvenientes de algunas técnicas de extracción y purificación

\begin{tabular}{|c|c|c|}
\hline Extracción & Ventajas & Inconvenientes \\
\hline $\begin{array}{l}\text { Extracción en } \\
\text { fase sólida }\end{array}$ & $\begin{array}{l}\star \quad \text { Ideal para muestras líquidas } \\
\star \quad \text { Menor gasto de disolvente }\end{array}$ & \# A veces extractos sucios \\
\hline $\begin{array}{l}\text { Extracción } \\
\text { líquido-líquido }\end{array}$ & $\begin{array}{ll}+ & \text { Simple } \\
* \quad \text { No precisa instrumentación }\end{array}$ & $\begin{array}{l}\text { \# Elevado consumo de disolventes } \\
\text { orgánicos } \\
\text { \# Disolventes orgánicos utilizados } \\
\text { perjudican el medioambiente }\end{array}$ \\
\hline $\begin{array}{l}\text { Extracción en } \\
\text { columnas de } \\
\text { inmunoafinidad }\end{array}$ & $\begin{array}{ll}* & \text { Ideal para muestras líquidas } \\
* & \text { Menor gasto de disolvente } \\
* & \text { Extractos limpios }\end{array}$ & $\begin{array}{l}* \text { Elevado coste de columnas de } \\
\text { inmunoafinidad } \\
* \quad \text { No reutilizables }\end{array}$ \\
\hline $\begin{array}{l}\text { Extracción con } \\
\text { fluidos } \\
\text { supercríticos }\end{array}$ & $\begin{array}{l}* \quad \text { Rápido }(30-60 \mathrm{~min}) \\
+\quad \text { Se puede conseguir alta selectividad } \\
+\quad \text { Baja cantidad disolventes }(5-10 \mathrm{ml}) \\
+\quad \text { No es necesaria la filtración posterior } \\
\text { del extracto }\end{array}$ & $\begin{array}{l}\# \quad \text { Tamaño muestra limitado }(<10 \mathrm{~g}) \\
\# \quad \text { Necesidad de modificadores para } \\
\text { mejorar la eficiencia de la extracción } \\
\# \quad \text { Coste elevado del equipo }\end{array}$ \\
\hline
\end{tabular}




\begin{tabular}{|c|c|c|}
\hline Ext & Ventajas & ivenientes \\
\hline $\begin{array}{l}\text { Extracción con } \\
\text { microondas }\end{array}$ & $\begin{array}{ll}+ & \text { Rápido }(15 \mathrm{~min}) \\
\star & \text { Bajo consumo disolventes }(15-40 \mathrm{ml}) \\
& \text { Sencillo }\end{array}$ & $\begin{array}{l}\# \text { El extracto obtenido debe ser } \\
\text { filtrado } \\
\# \text { Es necesaria la adicción de un } \\
\text { disolvente polar } \\
\# \text { Es necesaria la limpieza posterior } \\
\text { del extracto } \\
\star \quad \text { Coste moderado del equipo }\end{array}$ \\
\hline $\begin{array}{l}\text { Extracción } \\
\text { acelerada con } \\
\text { disolventes }\end{array}$ & $\begin{array}{ll}\# & \text { Rápido } \\
& \text { Baja cantidad disolventes }(15-40 \mathrm{ml}) \\
& \text { Control absoluto de los parámetros de } \\
\text { extracción (temperatura y presión, etc.) }\end{array}$ & $\begin{array}{ll}* \quad \text { Elevado coste de equipo } \\
\star \quad \text { Dependiente del tipo de matriz }\end{array}$ \\
\hline
\end{tabular}

En el caso concreto de la patulina, la técnica de extracción más empleada en los métodos de análisis comentados en el apartado 3.2, basados tanto en cromatografía como en $\mathrm{CE}$, ha sido la extracción líquido-líquido, empleando acetato de etilo como disolvente extractante universal y una purificación mediante una disolución de carbonato. Esta técnica presenta como desventajas su elevado coste, ya que se usa una gran cantidad de disolventes orgánicos, así como el tiempo empleado debido a los procesos de limpieza de los extractos con carbonato sódico, presentando además el inconveniente de que la disolución de carbonato sódico hace que la patulina se degrade ya que ésta no es estable en medio básico. Como alternativa se ha propuesto la extracción en fase sólida como método más rápido, más económico y menos contaminante para extraer la patulina en manzanas y zumos de manzana. Así, se ha utilizado un cartucho de copolímero macroporoso, lavado con bicarbonato sódico al 1\% y ácido acético al 1\%, eluyendo la patulina con $2 \%$ acetonitrilo en etil éter anhídro [67]. En otros casos se emplearon columnas de SPE con dos etapas de lavado [52] y la metodología más reciente emplea cartuchos de $\mathrm{C}_{18} \mathrm{y}$ una mezcla de hexano-acetato de etilo-acetona para eluir el analito [51], mejorando la exactitud del método ya que no es necesario emplear carbonato sódico que facilita la degradación de la patulina y ocasiona pérdidas en el proceso de extracción. 


\subsection{MICROEXTRACCIÓN LÍQUIDO-LÍQUIDO DISPERSIVA}

Uno de los objetivos de la Química Analítica moderna es la miniaturización, simplificación y automatización del procedimiento analítico completo, especialmente para acelerar la etapa de tratamiento de muestra, la cual es normalmente el cuello de botella del análisis. La introducción de la microextracción líquido-líquido dispersiva (con siglas en inglés, DLLME), introducida en 2006 por Assadi y col. [68], ha contribuido notablemente a la consecución de este objetivo, debido a su simplicidad, rapidez de operación y bajo consumo de disolventes y reactivos. Además este tipo de tratamiento de muestra permite obtener altos valores de recuperación, elevado factor de preconcentración y beneficios medioambientales con su uso. Debido a ello, la DLLME ha suscitado gran interés en los científicos que trabajan en el campo de la Química Analítica. Desde su introducción para la preconcentración de analitos orgánicos de muestras acuosas, un gran número de trabajos han conseguido una eficiente y rápida extracción de analitos orgánicos o inorgánicos mediante esta técnica de extracción $[69,70]$.

El principio de la DLLME está basado en dos pasos: (1) Inyección de una mezcla adecuada de los disolventes extractantes y dispersivos en el interior de la muestra acuosa que contiene los analitos: En este paso, el disolvente extractante es dispersado dentro de la muestra acuosa favoreciendo el paso de los analitos al mismo. Debido a que el área superficial existente entre el disolvente extractante y la fase acuosa es infinitamente grande, la transferencia de los analitos de la fase acuosa a la fase del extractante es muy rápida. De este modo, se consigue rápidamente el estado de equilibrio y la extracción es independiente del tiempo. Ésta es la ventaja más importante de este método. (2) Centrifugación de la disolución: Después de la centrifugación, los analitos estarán en la fase más densa, que habrá sedimentado, pudiendo ser extraídos fácilmente del resto de la muestra acuosa [71,72].

Los pasos de la microextracción líquido-líquido dispersiva están reflejados en la Figura 8.

En la DLLME, los principales factores que afectan a la eficiencia en la extracción son los siguientes:

Naturaleza del disolvente extractante 
Naturaleza del disolvente dispersivo

Volumen del disolvente extractante

Volumen del disolvente dispersivo

Volumen de muestra

Adición de sal

La selección de un disolvente extractante apropiado es el parámetro más determinante para el proceso de DLLME. Se suelen seleccionar disolventes orgánicos debido a que a menudo presenta una densidad más alta que el agua, a su capacidad de disolver a los compuestos de interés y a su buen comportamiento cromatográfico. Los hidrocarburos halogenados como clorobenceno, cloroformo, tetracloruro de carbono y tetracloroetileno, son normalmente seleccionados como disolventes extractantes debido a su alta densidad. El disolvente dispersivo debe ser miscible con el agua (disolvente polar) a la vez que debe ser parcialmente miscible con el disolvente extractante [68]. Como disolventes dispersivos pueden usarse acetona, acetonitrilo, metanol, etanol e isopropanol, entre otros. En relación con la extracción de micotoxinas, esta técnica se ha usado exclusivamente en la determinación de Ocratoxina A en vinos [73,74].

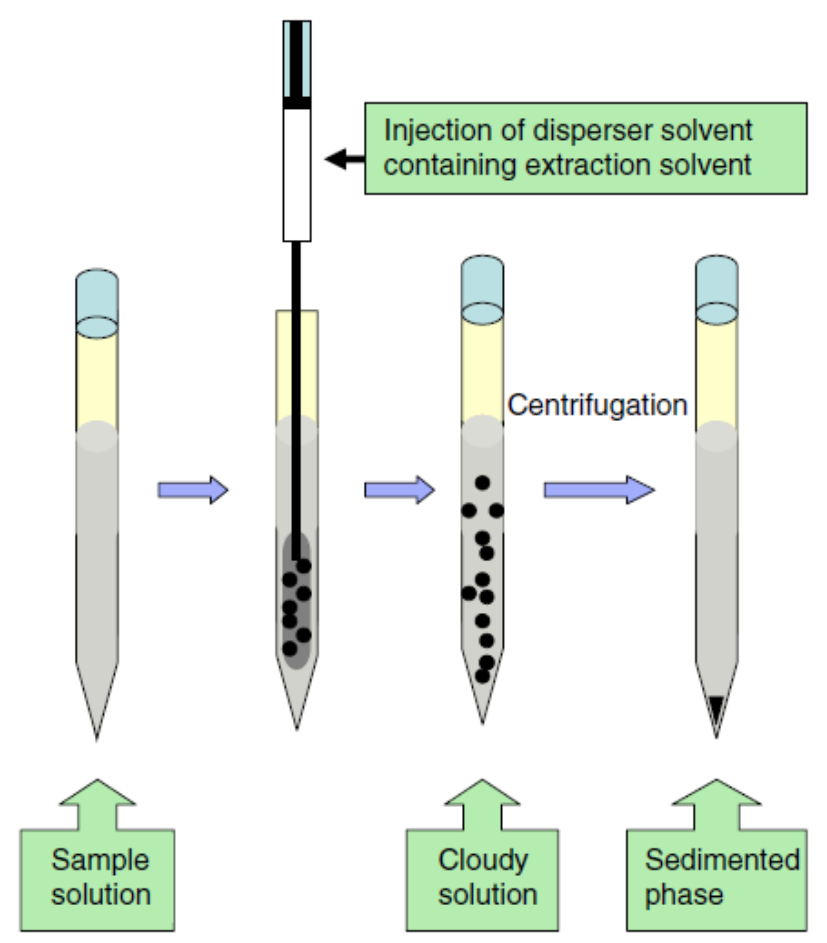

Figura 8. Esquema del procedimiento de DLLME 
En esta Memoria, se ha llevado a cabo la evaluación de la DLLME para la determinación de patulina en zumos de manzana, optimizando todas las variables implicadas en obtener una mayor eficacia en la extracción y empleando MEKC con detección UV-VIS como método de separación, basándonos en trabajos previos de determinación de patulina con MEKC usando otros sistemas de extracción [39,72]. De la bibliografía consultada acerca de las aplicaciones de la DLLME en el análisis de este compuesto, se puede concluir que este trabajo de investigación es el primero en utilizar esta técnica para la extracción de patulina en zumo de frutas. El método de determinación de patulina mediante DLLME-MEKC se ha caracterizado mediante el establecimiento de los parámetros de calidad usando muestras de zumo de manzana.

Para la puesta a punto de este método se han seguido las siguientes etapas:

Estudio de la separación electroforética en modo MEKC

Optimización de la DLLME

Establecimiento de las rectas de calibrado y límites de detección y determinación

Estudio de la precisión interdía e intradía

Estudio de la veracidad del método mediante ensayos de recuperación 


\section{REACTIVOS Y MATERIAL}




\section{REACTIVOS Y MATERIAL}

Todos los reactivos usados en la presente Memoria fueron de grado analítico y los disolventes de grado HPLC. Metanol, etanol, acetonitrilo (AcN), isobutanol, etiléter, acetato de etilo, dimetilformamida, cloruro sódico, dodecil sulfato sódico (SDS), ácido fosfórico (85\%), hidróxido sódico e hidrógeno fosfato disódico anhidro fueron suministrados por Panreac-Química (Madrid, España); ácido acético, cloroformo y tretacloroetileno por VWR BDH Prolabo (West Chester, Pensilvania, EEUU); disulfuro de carbono por Carlo Erba (Rodano, MI, Italia); tetrahidrofurano (THF) por Merck (Darmstadt, Alemania); tetraborato sódico, isopropanol por Sigma-Aldrich (St. Louis, MO, EEUU); tris(hidroximetil)aminometano (tris) y ácido clorhídrico por Merck (Darmstadt, Alemania).

El agua empleada en todo el trabajo fue agua ultrapura $\left(18.2 \mathrm{~m} \Omega \mathrm{cm}^{-1}\right)$ obtenida mediante el sistema Milli-Q (Millipore, Bedford, MA, EEUU).

Se utilizaron filtros de nylon de 0,2 $\mu \mathrm{m}$ (Supelco, Bellefonte, PA, EEUU) para la filtración del tampón electroforético, patrones y muestras introducidos en el equipo de CE. En el tratamiento de muestra se utilizaron tubos Falcon de 15 y 45 ml (VWR Iternational West Chester, PA, USA) y jeringas de 1 y 2 ml (BD Discardit, USA).

Se preparó una disolución madre de patulina a una concentración de 1000 $\mu \mathrm{g} \cdot \mathrm{mL}^{-1}$. Para ello, se disolvió la patulina suministrada en forma cristalina (SigmaAldrich) en AcN. Esta disolución se almacenó a $-20^{\circ} \mathrm{C}$ en frasco de vidrio topacio, preservándola de la luz. A partir de esta disolución madre se prepararon disoluciones intermedias $\left(100 \mu \mathrm{g} \cdot \mathrm{mL}^{-1}, 50 \mu \mathrm{g} \cdot \mathrm{mL}^{-1}\right.$ y $\left.10 \mu \mathrm{g} \cdot \mathrm{mL}^{-1}\right)$, disolviendo alícuotas de la anterior en AcN. Además, se preparó una disolución estándar de HMF a una concentración de $1000 \mu \mathrm{g} \cdot \mathrm{mL}^{-1}$, disolviendo el HMF suministrado en forma cristalina (Sigma-Aldrich) en AcN. Esta disolución se almacenó a $-20^{\circ} \mathrm{C}$ en frasco de vidrio topacio para preservarla de la luz. A partir de esta disolución estándar se prepararon disoluciones intermedias $\left(100 \mu \mathrm{g} \cdot \mathrm{mL}^{-1}\right)$, disolviendo una alícuota de la anterior en AcN. Finalmente, las disoluciones de trabajo de patulina y HMF, fueron preparadas diariamente eliminando el $\mathrm{AcN}$ mediante corriente de $\mathrm{N}_{2}$ y recomponiendo en agua Milli-Q acidificada con ácido acético a $\mathrm{pH} 4$, ya que la patulina es estable a $\mathrm{pH}$ ácido, mientras que a $\mathrm{pH}$ alcalino se hidroliza. 
El tampón electroforético consistía en una disolución acuosa $35 \mathrm{mM}$ de tetraborato sódico y $65 \mathrm{mM}$ de SDS, a pH 9 con un 5\% de AcN. Este tampón, conteniendo medio micelar, se preparaba disolviendo $1,259 \mathrm{~g}$ de borato sódico y 1,904 mg de SDS en $100 \mathrm{~mL}$ de agua Milli-Q con un 5\% v/v de AcN, ajustando el pH a 9 con disolución $\mathrm{HCl}$ 0,5 N. 


\section{INSTRUMENTACIÓN Y SOFTWARE}




\section{INSTRUMENTACIÓN Y SOFTWARE}

Las separaciones mediante CE se realizaron en un instrumento Agilent 7100 (Agilent Technologies, Waldbron, Alemania), equipado con detector de diodos en fila, operando a una longitud de onda de $276 \mathrm{~nm}$. El sistema empleado se muestra en la Figura 9. Las separaciones se llevaron a cabo primero en un capilar normal de sílice fundida, durante el estudio de variables que afectaban a la separación y, seguidamente, en un capilar del mismo material con celda de detección tipo burbuja con un camino óptico de $200 \mu \mathrm{m}$, durante la optimización del tratamiento de muestra y caracterización del método propuesto. Ambos capilares tenían una longitud total de 64,5 cm (longitud efectiva de $56 \mathrm{~cm}$ ) y un diámetro interno de $75 \mu \mathrm{m}$ (comercializados por Agilent Technologies).

Para las medidas de pH se utilizó un pH-metro Crison modelo pH 2000 (Barcelona, España). Para la preparación de las disoluciones estándar y muestras se utilizó un agitador Vórtex-2 Genie (Scientific Industries, Bohemia, NY, EEUU). Además, en el tratamiento de las muestras se empleó un sistema de evaporación de nitrógeno Evaporators EVA EC-S/EVA LS-S (VLM GmbH, Bielefeld, Alemania) y una centrífuga Model Universal 320R (Hettich, Tuttlingen, Alemania).

Para la obtención del zumo de manzana a partir de la fruta, utilizamos una licuadora (Multifruit Moulinex).

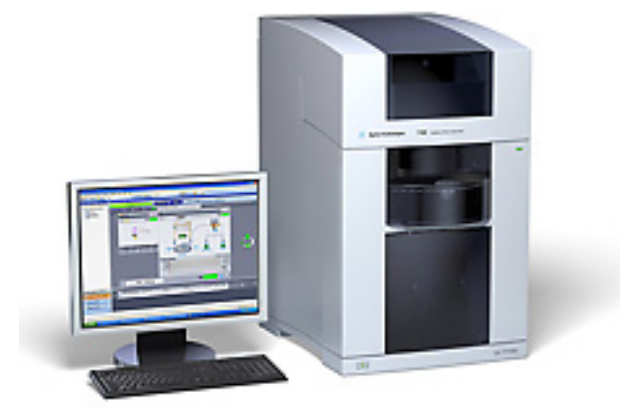

Figura 9. Equipo de CE acoplado a un detector UV-Vis

Los electroferogramas obtenidos se registraron y procesaron con el programa ChemStation, versión B.02.01. Asimismo, se utilizaron los programas Excel (Microsoft), versión 2007 y StatGraphics Plus 5.1 (Statistical Graphics, Rockville, MD, EEUU) para el posterior tratamiento de datos. 


\section{PROCEDIMIENTO ELECTROFORÉTICO}

\section{PROPUESTO}




\section{PROCEDIMIENTO ELECTROFORÉTICO PROPUESTO}

La separación de la patulina y su interferente (HMF) se llevó a cabo en un capilar burbuja de sílice fundida aplicando un voltaje de $15 \mathrm{kV}$ en modo normal y manteniendo el capilar a una temperatura de $35^{\circ} \mathrm{C}$. La inyección de la muestra se realizó en modo hidrodinámico aplicando 50 mbar durante $17 \mathrm{~s}$, lo que supone un volumen aproximado de $102,3 \mathrm{~nL}$ y un 3,59\% del volumen total del capilar.

En cuanto al empleo y conservación del capilar de separación, antes de ser empleados por primera vez, los capilares nuevos se acondicionaron con una disolución de $\mathrm{NaOH} 1 \mathrm{M}$ durante $20 \mathrm{~min}$, seguido de agua Milli-Q durante otros $10 \mathrm{~min}$ y finalmente con tampón electroforético $30 \mathrm{~min}$. Al principio de cada sesión de trabajo, el capilar se pre-acondicionaba haciendo pasar agua Milli-Q durante $3 \mathrm{~min}$, seguida de una disolución de $\mathrm{NaOH}$ 0,1 M durante 7 min, a continuación agua Milli-Q durante 1 min y, finalmente, tampón electroforético durante $20 \mathrm{~min}$. Después de cada análisis, el capilar se acondicionaba durante 2 min con $\mathrm{NaOH} 0,1 \mathrm{M}, 1$ min con agua Milli-Q y 2 min con tampón electroforético para mantener una adecuada repetitividad entre análisis. Con objeto de evitar el deterioro de las paredes del capilar por efecto del tampón así como la formación de depósitos de sales, al final de cada sesión se lavaba con agua, durante 5 minutos a 5 bares de presión y, posteriormente, se pasaba una corriente de aire en las mismas condiciones que la corriente de agua. 


\section{TRATAMIENTO DE MUESTRA}




\section{PROCEDIMIENTO DE TRATAMIENTO DE MUESTRA MEDIANTE DLLME}

Como tratamiento de muestra se decidió emplear la microextracción líquidolíquido dispersiva (DLLME) con objeto de preconcentrar los analitos y limpiar la muestra.

A continuación se describe el tratamiento de muestra propuesto, recogido asimismo en la Figura 10:

- A partir de manzanas compradas en un supermercado local, peladas y cortadas en trozos, se prepara zumo mediante una licuadora de uso doméstico

- Se centrifugan $20 \mathrm{~mL}$ de zumo a $9000 \mathrm{rpm}$ durante $10 \mathrm{~min}$, para separar las partículas sólidas presentes en el mismo

- Se toman $5 \mathrm{~mL}$ de zumo en un tubo Falcon de $15 \mathrm{~mL}$. Cuando se trate de zumo de manzana comercial ya envasado se añaden estos $5 \mathrm{~mL}$ directamente, sin necesidad de llevar a cabo el paso previo de centrifugación

- $\quad$ Se añade $20 \%$ de $\mathrm{NaCl}(1 \mathrm{~g}$ de $\mathrm{NaCl})$

- Se inyecta rápidamente la mezcla del disolvente de dispersión $(1000 \mu \mathrm{L}$ de isopropanol) y del disolvente de extracción (1000 $\mu \mathrm{L}$ cloroformo)

- Se agita vigorosamente durante $20 \mathrm{~s}$

- $\quad$ Se centrifuga a $5000 \mathrm{rpm}$ durante $5 \mathrm{~min}$

- Una vez que se ha producido la separación de fases, se extrae con ayuda de una jeringa la fase más densa, que contiene a la patulina

- Se seca con corriente de nitrógeno

- $\quad$ Se recompone en 0,5 mL agua a pH 4 (acidificada con ácido acético)

- $\quad$ Se filtra (con un filtro de $0,2 \mu \mathrm{m}$ )

- $\quad$ Finalmente se analiza mediante MEKC con detección UV-Vis

En este caso, con el tratamiento DLLME descrito, el factor de preconcentración es de 10 veces. 


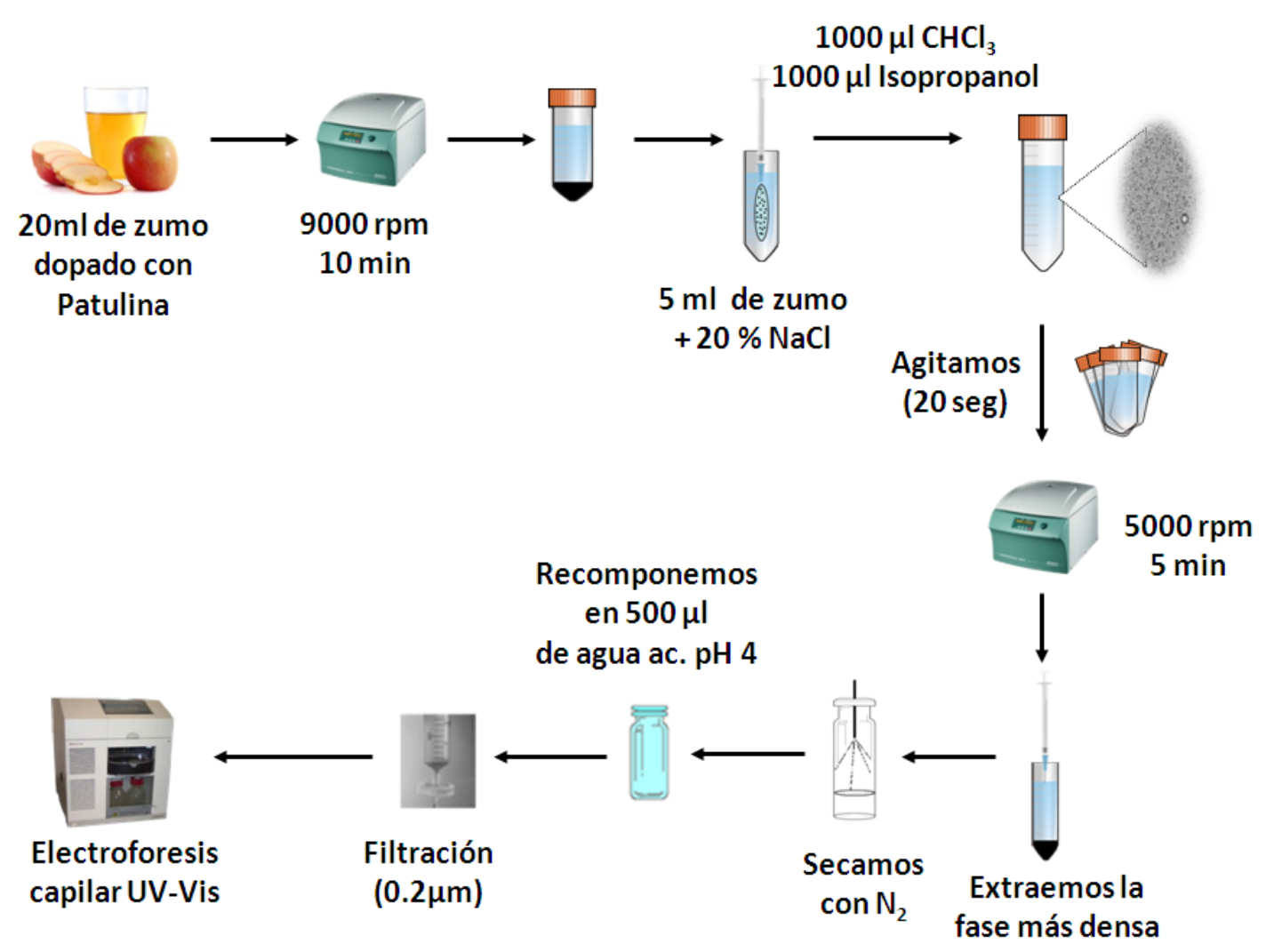

Figura 10. Esquema del tratamiento de muestra para la determinación de patulina en zumo de manzana por el método propuesto

La Figura 11 presenta los electroferogramas obtenidos para una muestra de zumo de manzana sin fortificar y el obtenido para una muestra del mismo zumo al que se ha añadido $20 \mu \mathrm{g} \cdot \mathrm{L}^{-1}$ de patulina.

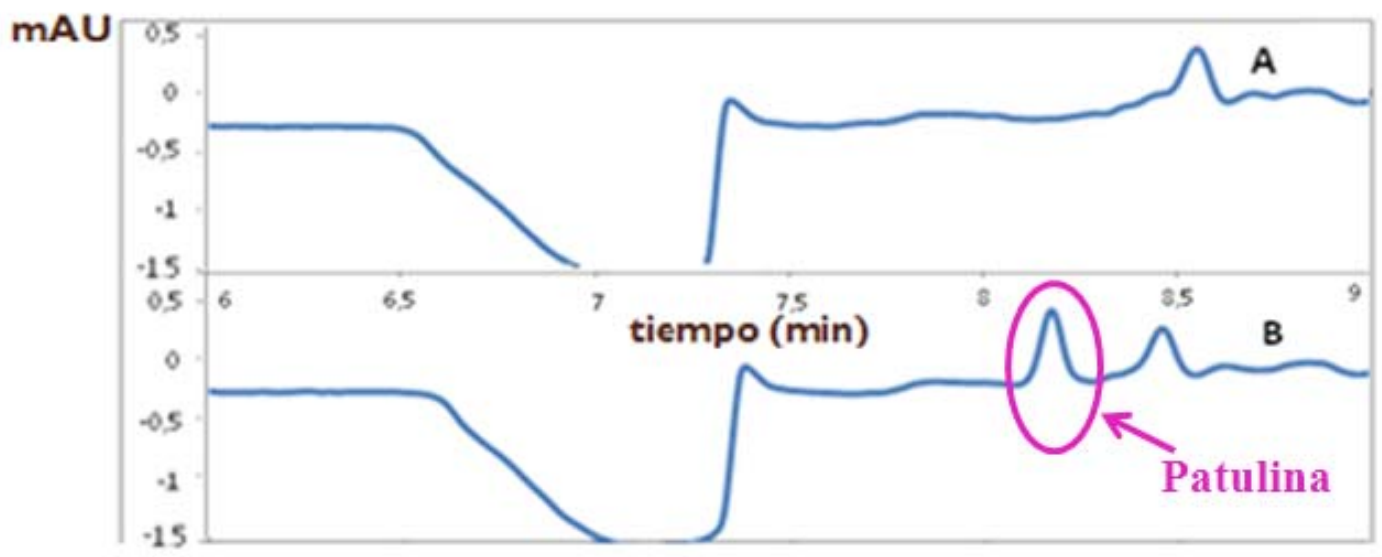

Figura 11. Electroferogramas obtenidos en las condiciones óptimas para: A) Blanco de zumo de manzana, B) Zumo de manzana fortificado con $20 \mu \mathrm{g} \cdot \mathrm{L}^{-1}$.

El análisis del blanco del zumo de manzana mostró que no había presencia de patulina en éste ni sustancias que comigraran (Figura 11). 


\section{RESULTADOS Y DISCUSIÓN}




\section{RESULTADOS Y DISCUSIÓN}

\subsection{ESTUDIO DE LA SEPARACIÓN ELECTROFORÉTICA}

Basándonos en trabajos previos de determinación de patulina mediante MECK, aplicando otros procedimientos de tratamiento de muestra [41,60], en este trabajo decidimos confirmar la selección de variables que influyen en la separación electroforética, previamente a la optimización del tratamiento de muestra mediante DLLME. Se llevó a cabo el estudio de cada una de las variables implicadas en el proceso, con objeto de obtener unos valores óptimos que proporcionen la mejor separación electroforética en términos tanto de intensidad de la señal analítica como de resolución entre la patulina y HMF, su principal interferente. Todo ello en el menor tiempo de análisis posible y manteniendo la corriente del capilar siempre por debajo de $120 \mu \mathrm{A}$.

Las condiciones de partida empleadas para la optimización fueron las siguientes: capilar de sílice fundida de una longitud de $64,5 \mathrm{~cm}$ y $75 \mu \mathrm{m}$ de d.i., aplicando un voltaje de $15 \mathrm{kV}$ y una temperatura de $35^{\circ} \mathrm{C}$. Se empleó una disolución de trabajo mezcla de patulina e HMF de 5 y $50 \mu \mathrm{g} \cdot \mathrm{ml}^{-1}$, respectivamente. Para ello se tomaban los volúmenes adecuados de las disoluciones intermedias de cada una y se llevaban al mismo vial. Seguidamente, se le aplicaba una corriente de $\mathrm{N}_{2}$ para eliminar el disolvente $(\mathrm{AcN})$ y se reconstituía en agua acidificada $\mathrm{pH} 4$, ya que hay estudios en bibliografía que indican que este medio de reconstitución es el ideal para la patulina, debido a que es químicamente lábil en medio alcalino [61]. Finalmente, esta disolución se introducía mediante inyección hidrodinámica durante 15 segundos a una presión de 50 mbar.

A continuación se comentan cada uno de los parámetros estudiados.

\subsubsection{ESTUDIO DEL pH DE LA SEPARACIÓN}

El pH es una variable crítica en $\mathrm{CE}$, por lo que fue el primer parámetro estudiado. El estudio se realizó en un intervalo de $\mathrm{pH}$ comprendido entre 3 y 12. Para ello, la disolución tampón empleada fue fosfato sódico $30 \mathrm{mM}$ con $60 \mathrm{mM}$ de SDS. En la Figura 12-a podemos observar cómo influye el $\mathrm{pH}$ en el área de pico de la patulina. 
Como resultado del estudio se observó que, a medida que aumentaba el $\mathrm{pH}$, aumentaba la señal hasta $\mathrm{pH}$ 9, y a partir de ese valor disminuía. Para $\mathrm{pH} 3$ no se obtuvo ninguna señal.

Hay que indicar que en todo este rango de $\mathrm{pH}$, la patulina permanece neutra, pues tiene un pKa de alrededor de 12.

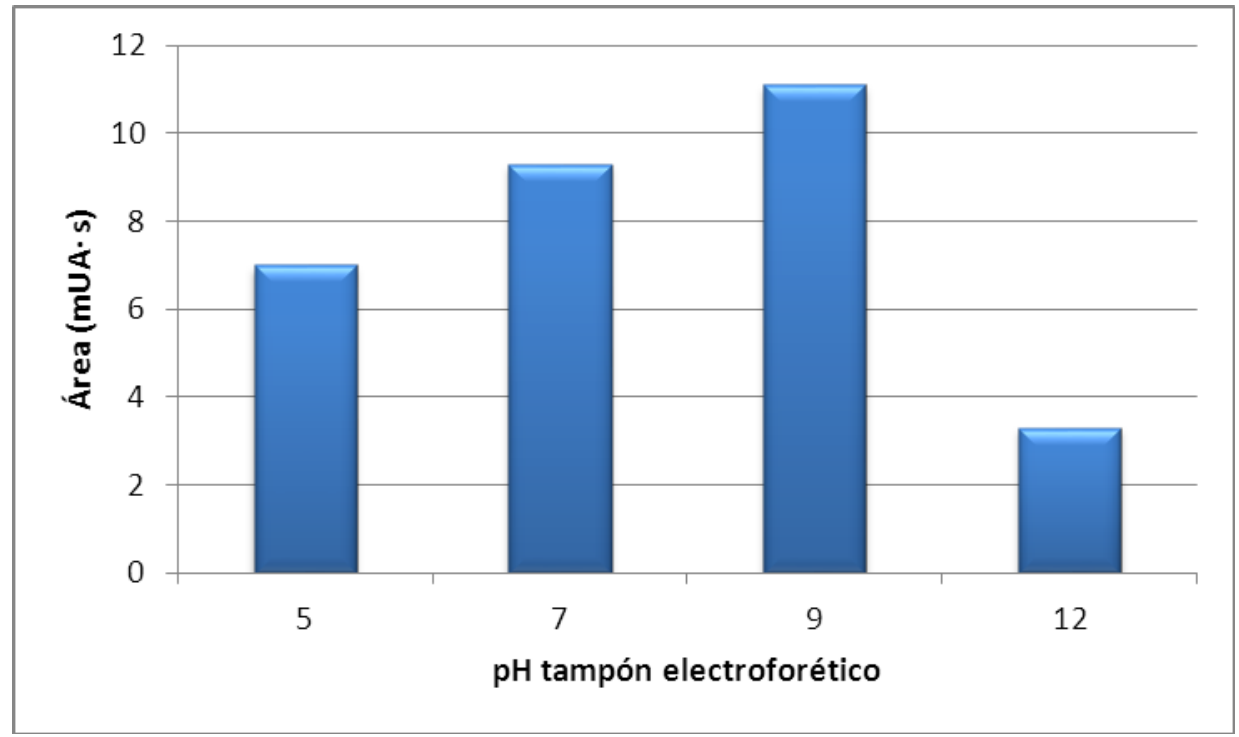

Figura 12-a. Influencia del pH sobre el área de pico

Por otra parte, a medida que aumentábamos el pH disminuía el tiempo de análisis varios minutos como consecuencia del aumento del EOF.

En base a los resultados anteriores, se decidió estudiar más detalladamente el rango alrededor del $\mathrm{pH}$ 9. Los resultados obtenidos en este estudio se muestran en la Figura 12-b. 


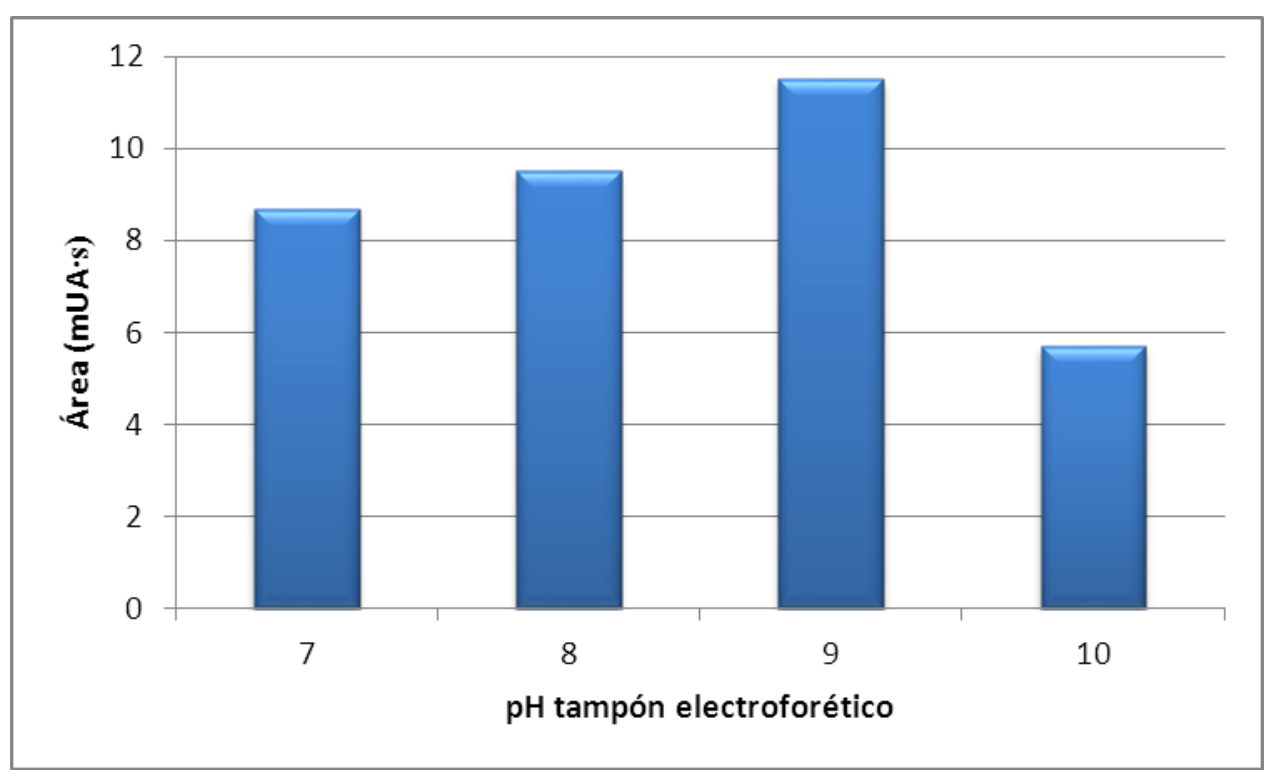

Figura 12 -b. Influencia del pH sobre el área de pico

Los electroferogramas obtenidos para este último rango de $\mathrm{pH}$ estudiado quedan reflejados en la Figura 12-c.

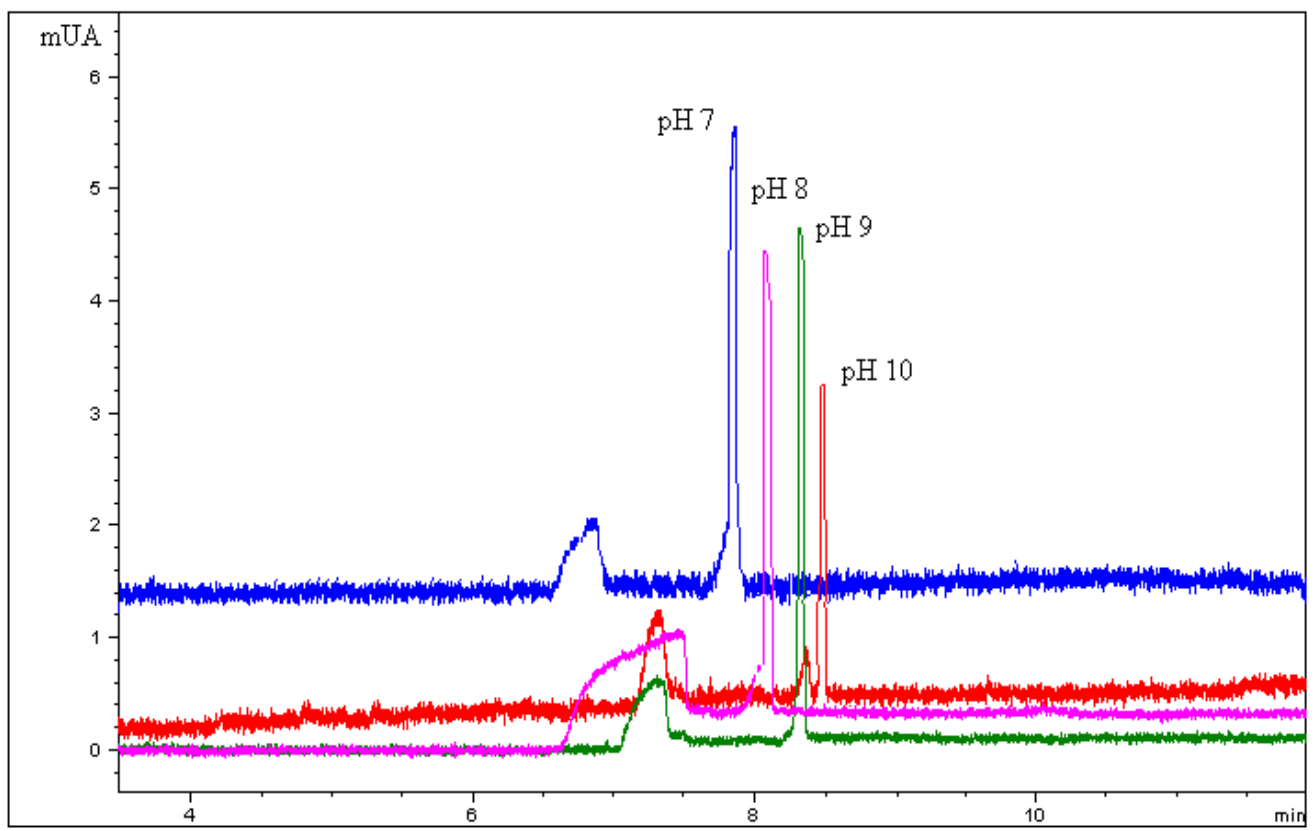

Figura 12-c. Electroferogramas obtenidos en el estudio del pH de la disolución tampón

Teniendo en cuenta los resultados obtenidos, se seleccionó finalmente un $\mathrm{pH}$ de 9 por ser el valor de $\mathrm{pH}$ al cual el área de pico correspondiente es mayor, en un tiempo de análisis adecuado de menos de 9 minutos. 


\subsubsection{ESTUDIO DE LA NATURALEZA Y CONCENTRACIÓN DEL TAMPÓN ELECTROFORÉTICO}

Una vez optimizado el $\mathrm{pH}$ y fijado el valor de 9 se estudió la naturaleza del tampón empleado como electrolito de separación. Los distintos tampones elegidos para este estudio fueron: tetraborato sódico, fosfato sódico y tris. La concentración empleada para todas las disoluciones fue $30 \mathrm{mM}$ y a todas se les añadió $60 \mathrm{mM}$ de SDS. Los electroferogramas obtenidos para los tampones borato, fosfato y tris se muestran en la Figura 13.

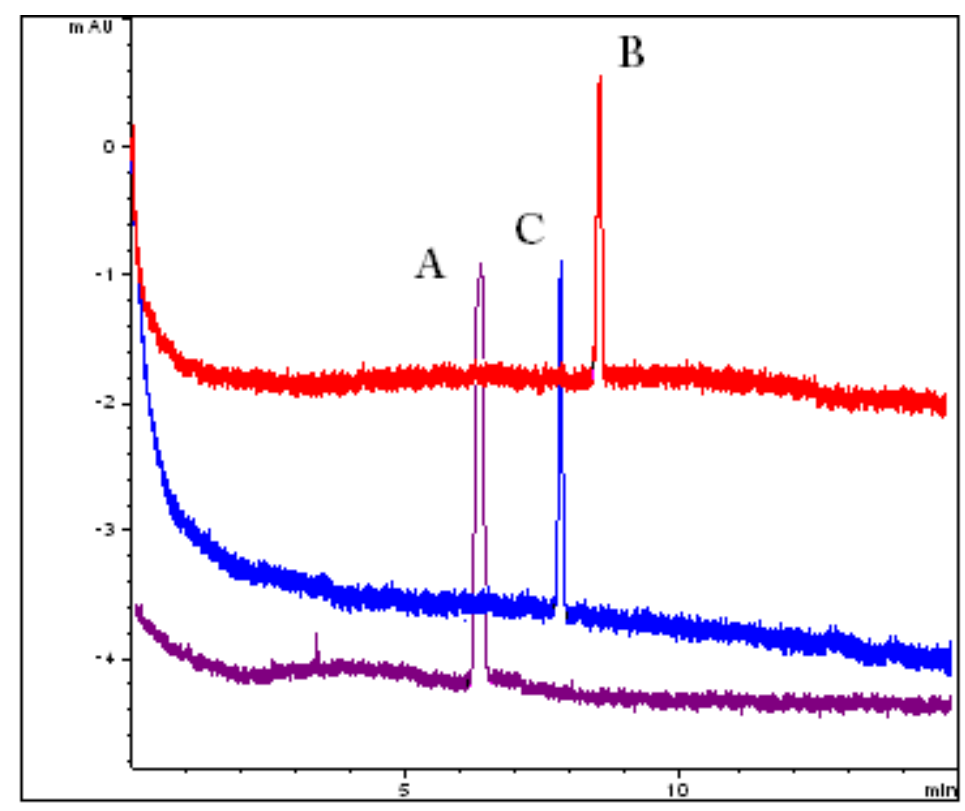

Figura 13. Electroferogramas obtenidos en el estudio de la naturaleza de la disolución tampón: A) Tetraborato sódico, B) Tris, C) Fosfato sódico

Los resultados muestran que el empleo de tetraborato sódico o fosfáto sódico como tampones electroforéticos proporcionan los mejores resultados. Sin embargo, con el tetraborato sódico el tiempo de migración es menor y el área de pico ligeramente mayor, por tanto, éste ha sido el seleccionado para experiencias posteriores.

El criterio adoptado para seleccionar la concentración del tampón electroforético más adecuada fue conseguir la mayor sensibilidad en la señal del analito imponiendo la limitación de que el tiempo de análisis no fuera excesivamente largo. 
En el estudio se probaron los niveles de concentración del tampón que se señalan a continuación: 10, 20, 35 y 50 mM. En la Figura 14-a, podemos ver los resultados obtenidos en términos de señal analítica para las diferentes concentraciones de tampón.

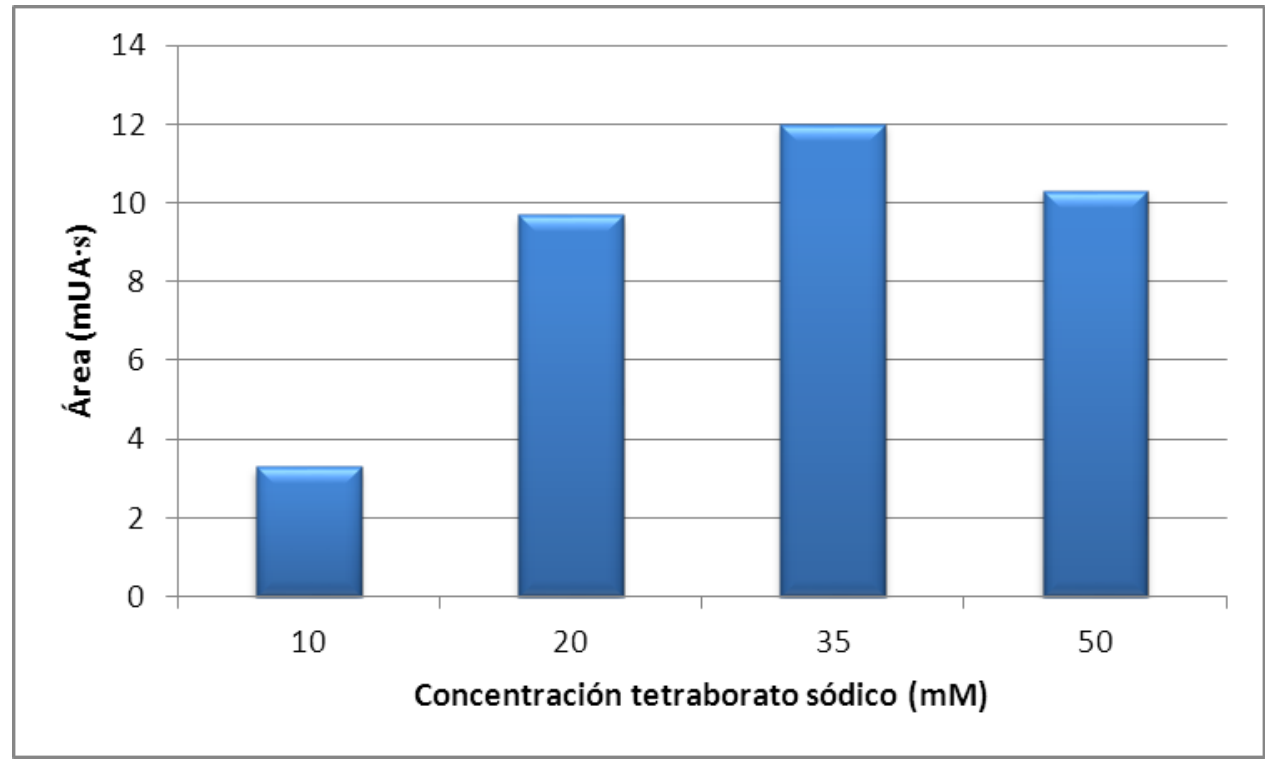

Figura 14-a. Influencia de la concentración de tetraborato sódico sobre el área de pico

Los electroferogramas obtenidos para las diferentes concentraciones de tampón estudiadas están representados en la Figura 14-b.

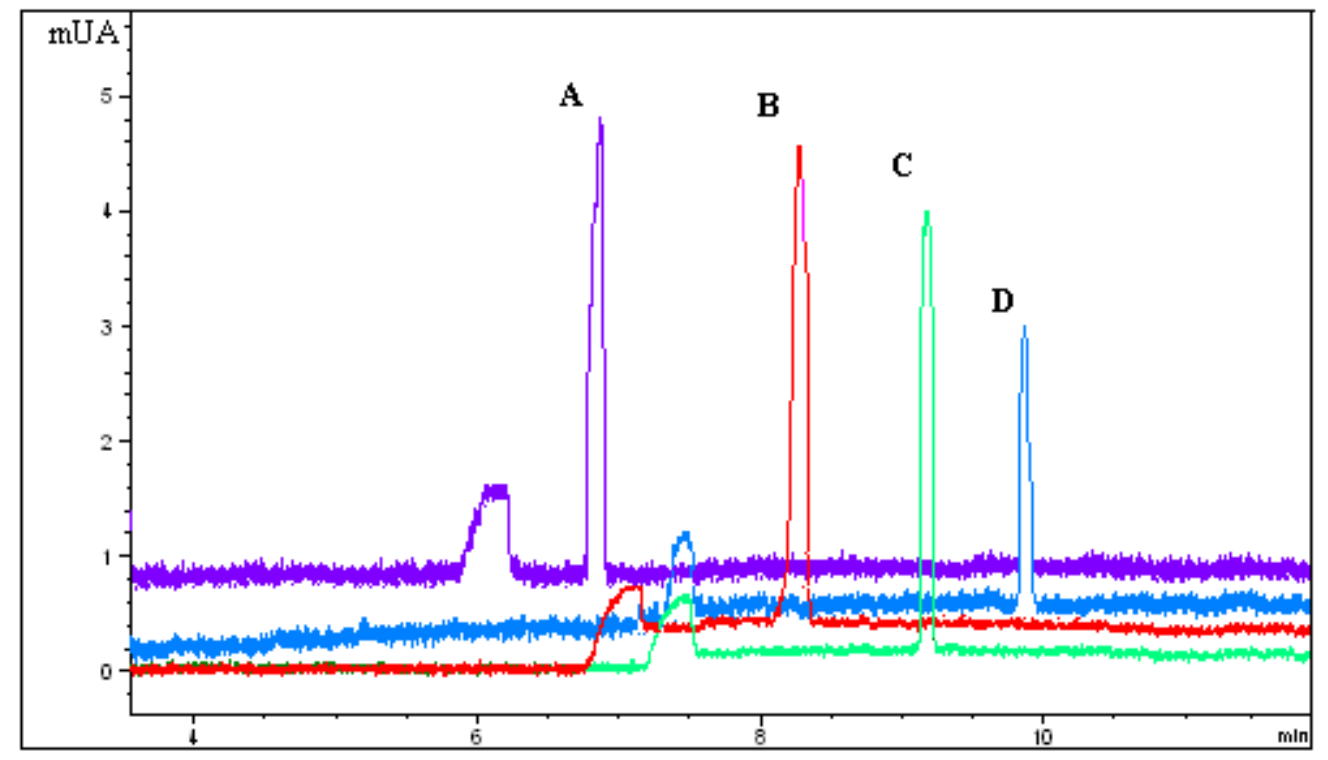

Figura 14-b. Electroferogramas obtenidos en el estudio de la concentración de la disolución tampón: A) $50 \mathrm{mM}$, B) $35 \mathrm{mM}$, C) $20 \mathrm{mM}$, D) $10 \mathrm{mM}$ 
Para altas concentraciones de tetraborato sódico, la corriente del sistema aumentaba considerablemente, por encima de los $110 \mu \mathrm{A}$, por lo que se observó que la concentración que ofrecía mejores resultados era la de $35 \mathrm{mM}$.

\subsubsection{ESTUDIO DE LA CONCENTRACIÓN DE SDS}

La patulina es una molécula neutra en prácticamente todo el rango de $\mathrm{pH}$, al ser su $\mathrm{pK}_{\mathrm{a}}$ próximo a 12, por tanto, para poder analizarla al $\mathrm{pH}$ seleccionado fue imprescindible añadir un surfactante al medio electroforético para que fuera posible la separación gracias a la formación de micelas, empleando como modo de separación la cromatografía capilar electrocinética micelar (MEKC). Igualmente esto se justifica si se considera la separación respecto del HMF, también neutro. El surfactante seleccionado para el estudio fue dodecil sulfato sódico (SDS). El hecho de que la disolución tampón contenga micelas con carga negativa, hace que éstas sean atraídas por el ánodo, aunque globalmente migran hacia el cátodo impulsadas por el elevado EOF a $\mathrm{pH}$ básico, favoreciendo de este modo la separación de los analitos.

Para seleccionar la concentración de medio micelar idónea, se estudiaron concentraciones crecientes de $\operatorname{SDS}(20,35,50,65$ y $80 \mathrm{mM})$ en el tampón de separación, siempre por encima de la CMC de este surfactante $(8,2 \mathrm{mM}$ en agua pura a $25^{\circ} \mathrm{C}$ ). La Figura 15 muestra la influencia de la concentración de SDS sobre el área de pico y el tiempo de análisis. Si observamos esta Figura 15, podemos ver que la situación óptima se tiene para una concentración de SDS de $65 \mathrm{mM}$, ya que al aumentar la concentración de micelas aumenta la viscosidad del medio, incrementando el tiempo de migración de la patulina que pasa de 5,5 min cuando la concentración de SDS es $20 \mathrm{mM}$ a 9 min cuando la concentración de SDS es $80 \mathrm{mM}$. 




Figura 15. Influencia de la concentración de SDS sobre el área de pico y el tiempo de análisis.

Por otro lado, se observó que al aumentar la concentración de SDS en el tampón electroforético, la corriente también aumentaba, lo cual era un factor limitante, de modo que se seleccionó una concentración de $65 \mathrm{mM}$ de SDS, siendo la corriente de aproximadamente $100 \mu \mathrm{A}$, para experiencias posteriores.

\subsubsection{ESTUDIO DEL VOLTAJE DE SEPARACIÓN}

Con objeto de disminuir el tiempo total de análisis, sin aumentar excesivamente la corriente, se modificó el voltaje de separación aplicado en modo normal positivo entre 10 y $25 \mathrm{kV}$. Se observó que, a medida que aumentó el voltaje, el tiempo de análisis se redujo considerablemente (Figura 16). Por otro lado, la resolución entre el pico del HMF y el de la patulina también aumentaba, sin embargo, las áreas y alturas de pico disminuyeron, por lo que se seleccionó como solución de compromiso un valor de $15 \mathrm{kV}$. A este voltaje la corriente se mantenía en un valor aproximado de $80 \mu \mathrm{A}$, mientras que a voltajes superiores la corriente superaba notablemente los $100 \mu \mathrm{A}$. 


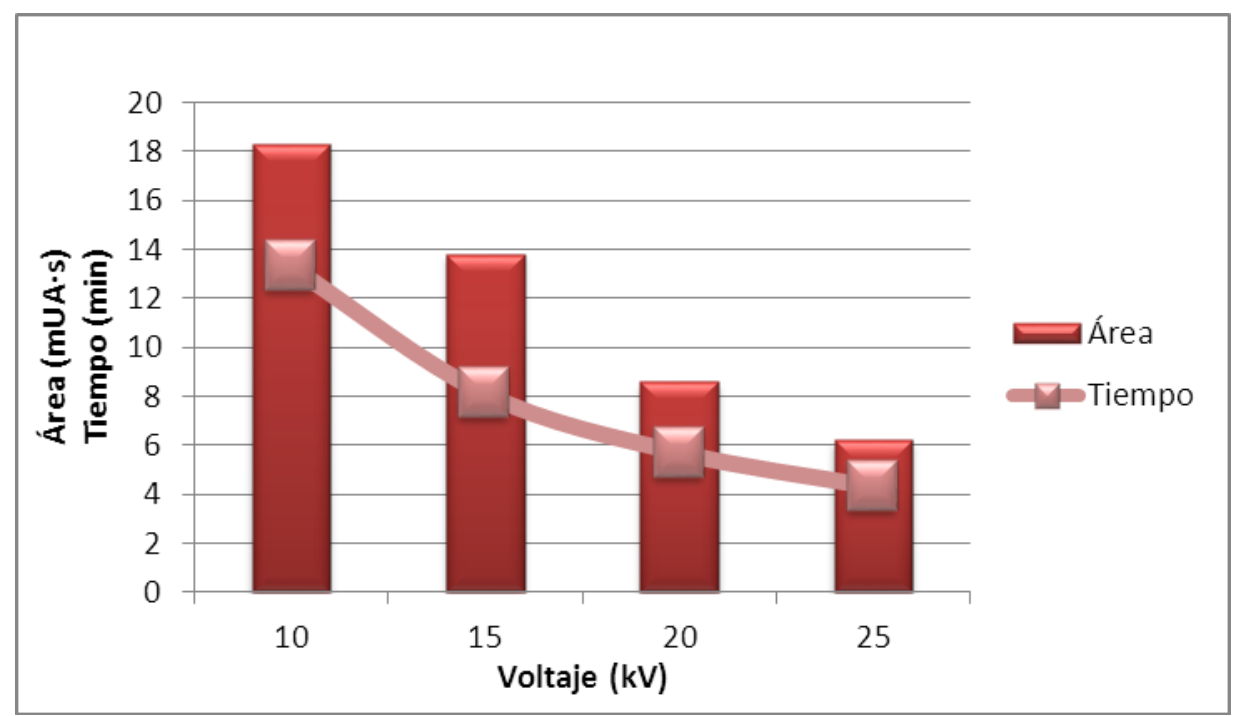

Figura 16. Influencia del voltaje aplicado sobre el área de pico y el tiempo de análisis

\subsubsection{ESTUDIO DE LA TEMPERATURA DEL CAPILAR}

El estudio de la temperatura (T) del capilar se realizó a $15,25,35$ y $45^{\circ} \mathrm{C}$. Según se observa en la Figura 17, la variación de la temperatura no influía significativamente en la intensidad de los picos, pero sí en el tiempo de análisis y la forma de pico, ya que a mayor temperatura se tenía menor tiempo de análisis y picos ligeramente más estrechos. Así, se seleccionó la temperatura de $35^{\circ} \mathrm{C}$, ya que a temperaturas mayores aumentaba la irreproducibilidad del análisis, y además aumentaba la corriente del capilar.

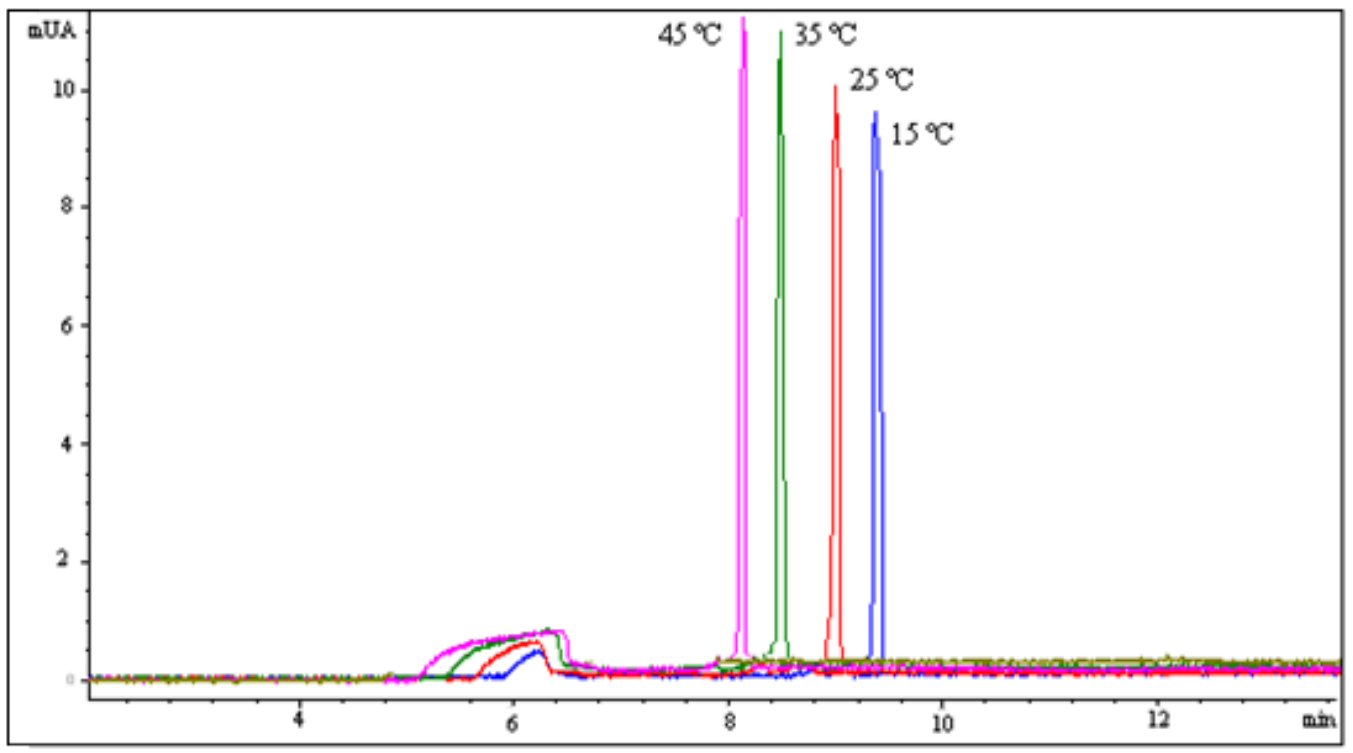

Figura 17. Influencia de la $\mathrm{T}^{\mathrm{a}}$ del capilar sobre la forma de pico y el tiempo de análisis 


\subsubsection{ESTUDIO DEL VOLUMEN DE INYECCIÓN}

El volumen de inyección puede seleccionarse controlando la presión de inyección o el tiempo de inyección. Puesto que el equipo de electroforesis utilizado nos permite trabajar hasta una presión de 50 mbar, fijamos este valor y se incrementó el tiempo de inyección, lo que implica un mayor volumen de muestra inyectado.

Tabla 6. Volumen de muestra y porcentaje de capilar llenado en función de diferentes tiempos de inyección.

\begin{tabular}{|c|c|c|}
\hline Tiempo de inyección (s) & $\begin{array}{c}\text { Volumen de muestra } \\
\text { inyectado (nL) }\end{array}$ & Llenado del capilar (\%) \\
\hline 12 & 72,2 & 2,54 \\
\hline 15 & 90,3 & 3,17 \\
\hline 17 & 102,2 & 3.59 \\
\hline 20 & 120,4 & 4,23 \\
\hline
\end{tabular}

La Figura 18 muestra la influencia del tiempo de inyección en la intensidad de la señal analítica. En ésta se puede observar que el mejor resultado se obtenía para un tiempo de inyección de 17 segundos.

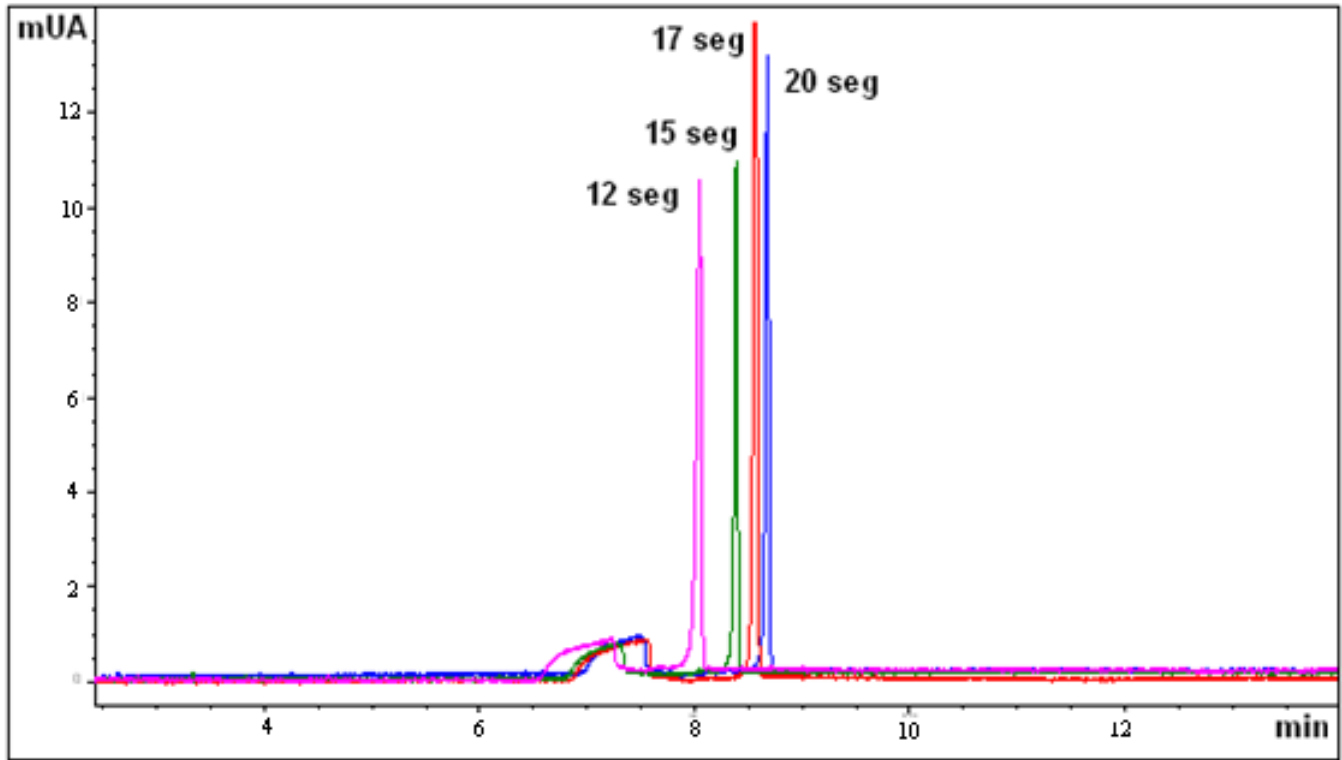

Figura 18. Electroferogramas obtenidos variando el tiempo de inyección

Todo el estudio de los factores influyentes en la separación electroforética se llevó a cabo en un capilar normal de sílice fundida. A continuación, la optimización del 
tratamiento de muestra se realizó en un capilar burbuja con paso óptico aumentado, también de sílice fundida, que permitía aumentar la sensibilidad del método, llegándose a obtener el doble de intensidad en las señales, respecto del primer capilar utilizado.

A continuación, se muestra un resumen de la optimización del método electroforético, indicando los valores seleccionados para cada uno de los parámetros estudiados (Tabla 7).

Tabla 7. Resumen de los valores óptimos de la separación electroforética

\begin{tabular}{|l|l|}
\hline Capilar & $\begin{array}{l}\text { Burbuja de sílice fundida. Dimensiones. } 64,5 \mathrm{~cm} \text { de longitud, } \\
75 \mu \mathrm{m} \text { de d.i., con una longitud efectiva de } 56 \mathrm{~cm}\end{array}$ \\
\hline $\mathrm{pH}$ & 9 \\
\hline Tampón electroforético & tetraborato sódico $35 \mathrm{mM}$, SDS $65 \mathrm{mM}$ \\
\hline Medio reconstitución de la muestra & agua pH 4, acidificada con ác. acético \\
\hline Voltaje de separación & $15 \mathrm{kV}$ \\
\hline Temperatura & $35^{\circ} \mathrm{C}$ \\
\hline Inyección & Hidrodinámica, $17 \mathrm{~s} \mathrm{a} 50 \mathrm{mbar}(\approx 100 \mathrm{~nL})$ \\
\hline
\end{tabular}

La Figura 19 corresponde al electroferograma obtenido empleando las condiciones óptimas de separación fijadas previamente para disolución patrón.

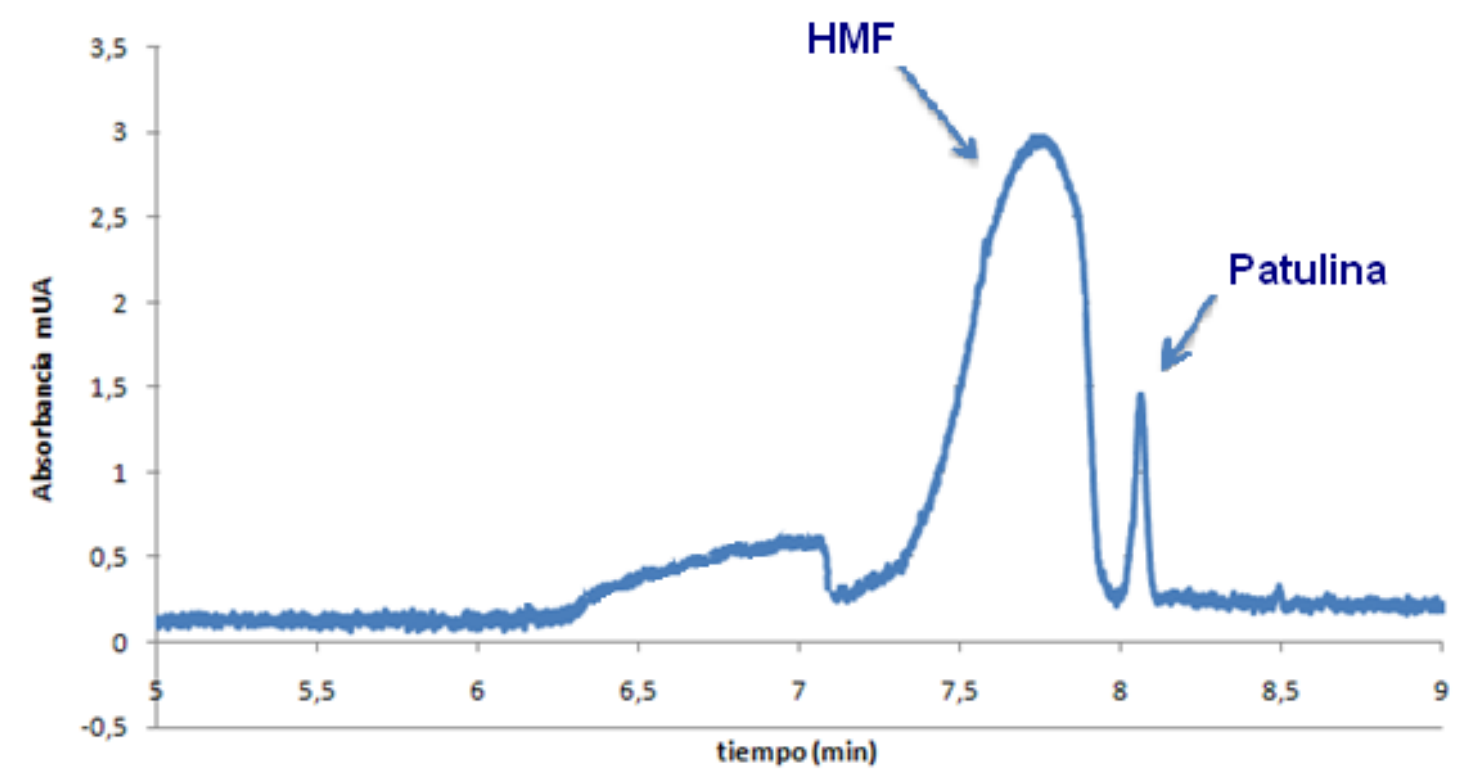

Figura 19. Electroferograma obtenido en condiciones de separación óptimas para 200 $\mu \mathrm{g} \mathrm{L}{ }^{-1}$ de patulina y $5 \mathrm{mg} \mathrm{L}^{-1}$ de HMF. 


\subsection{OPTIMIZACIÓN DE LA MICROEXTRACCIÓN LÍQUIDO-LÍQUIDO DISPERSIVA}

Los factores que afectan al proceso de extracción, y que deberán ser optimizados, son: la naturaleza y el volumen del disolvente dispersivo, la naturaleza y el volumen del disolvente extractante, el contenido salino en la muestra, el volumen de muestra y el tiempo de centrifugación.

Como criterio para seleccionar los valores óptimos, se estudió la eficacia del proceso de DLLME, calculada mediante los porcentajes de recuperación al aplicar el método $\mathrm{MEKC}$, estimados como cociente entre el área del pico de patulina obtenida para la muestra fortificada antes del tratamiento y la obtenida para una muestra libre de analito y fortificada al final del tratamiento con la misma concentración que debería haber en el extracto reconstituido que se va a inyectar en el sistema electroforético si el rendimiento de la extracción fuera del $100 \%$. De este modo es posible evaluar las pérdidas sufridas mediante el proceso de tratamiento de muestra.

La optimización de todas las variables implicadas se llevó a cabo en modo univariante. En todos los estudios, cada punto de la optimización era la media de cuatro valores (dos réplicas experimentales y dos instrumentales).

Todos los ensayos se realizaron en $5 \mathrm{~mL}$ de muestra fortificados con $20 \mu \mathrm{g} \mathrm{L}^{-1}$ de patulina y las condiciones de partida empleadas para la optimización fueron las siguientes: $700 \mu \mathrm{L}$ de cloroformo como disolvente extractante, $900 \mu \mathrm{L}$ de AcN como disolvente dispersivo, agitación manual de 20 segundos, centrifugación durante dos minutos a $5000 \mathrm{rpm}$ y posterior secado con corriente de $\mathrm{N}_{2}$ y recomposición en 0,5 ml de agua a $\mathrm{pH} 4$.

\subsubsection{OPTIMIZACIÓN DE LA NATURALEZA DEL DISOLVENTE EXTRACTANTE}

Se seleccionaron para el estudio cloroformo, tetracloroetileno, sulfuro de carbono, diclorometano y tetracloruro de carbono. 
Cualitativamente se observaba una mejor separación de las fases utilizando cloroformo como extractante, además éste ofrecía las mejores recuperaciones y el menor rango de error aleatorio, como puede observarse en la Figura 20.

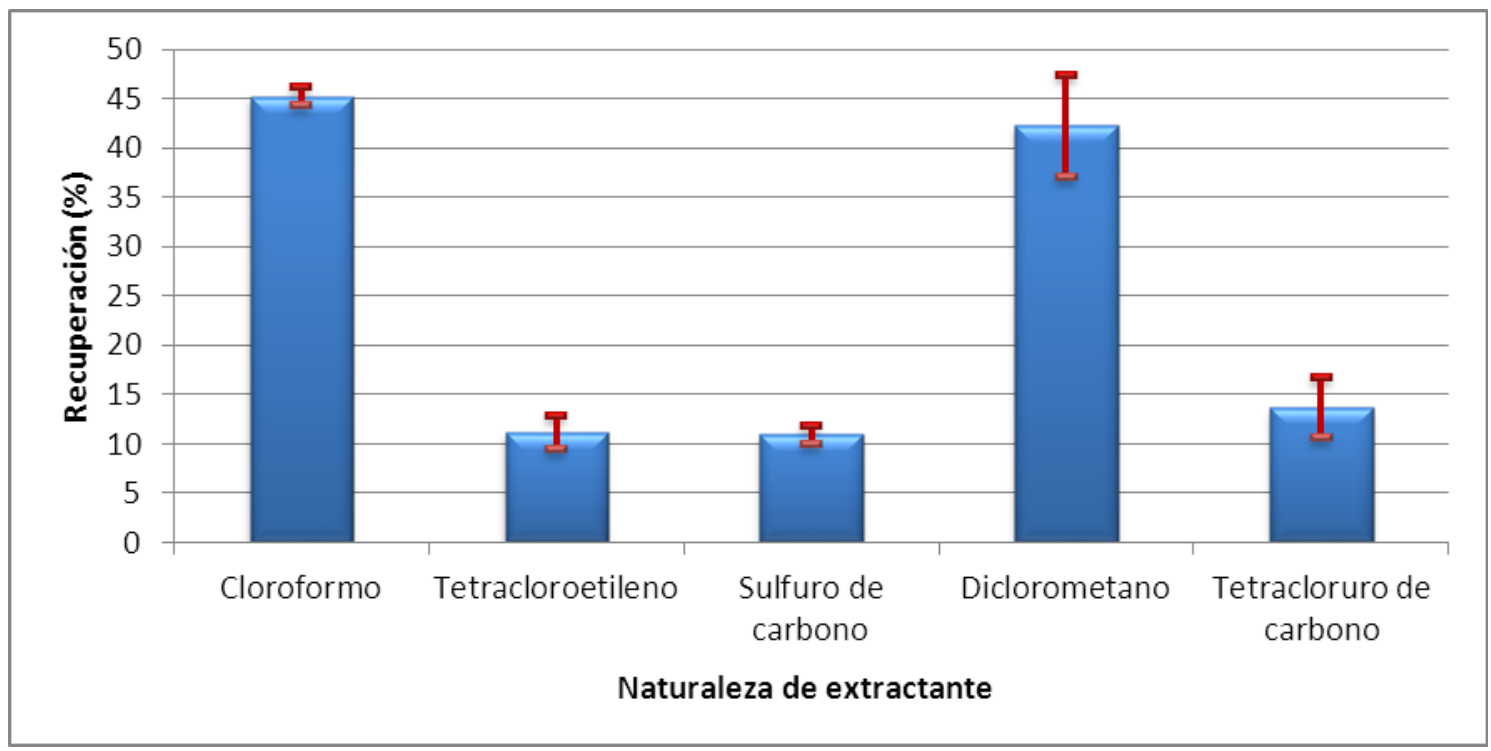

Figura 20. Influencia de la naturaleza del extractante en el porcentaje de recuperación

\subsubsection{OPTIMIZACIÓN DE LA NATURALEZA DEL DISOLVENTE DISPERSIVO}

Seguidamente se estudió la naturaleza del disolvente dispersivo, estudiando los siguientes disolventes: acetonitrilo, dimetilformamida, tetrahidrofurano (THF), isopropanol, etanol, isobutanol y etiléter.

En la Figura 21 se recogen los resultados de las recuperaciones obtenidas. Como puede observarse, los mejores porcentajes de recuperación se obtuvieron utilizando como dispersivo THF, isopropanol e isobutanol. Sin embargo, se optó finalmente por utilizar isopropanol, ya que cuando utilizábamos THF la variabilidad en los resultados era bastante mayor que para los otros dos: $11 \%$ con THF, $2,3 \%$ con isopropanol y $4,3 \%$ con isobutanol. Por otro lado, cuando utilizábamos isobutanol, se producía una inversión en las fases, de modo que, la fase acuosa pasaba a ser la más densa, mientras que la orgánica la menos densa, quedando esta última en la parte superior del tubo Falcon y dificultando, por tanto, su separación. 


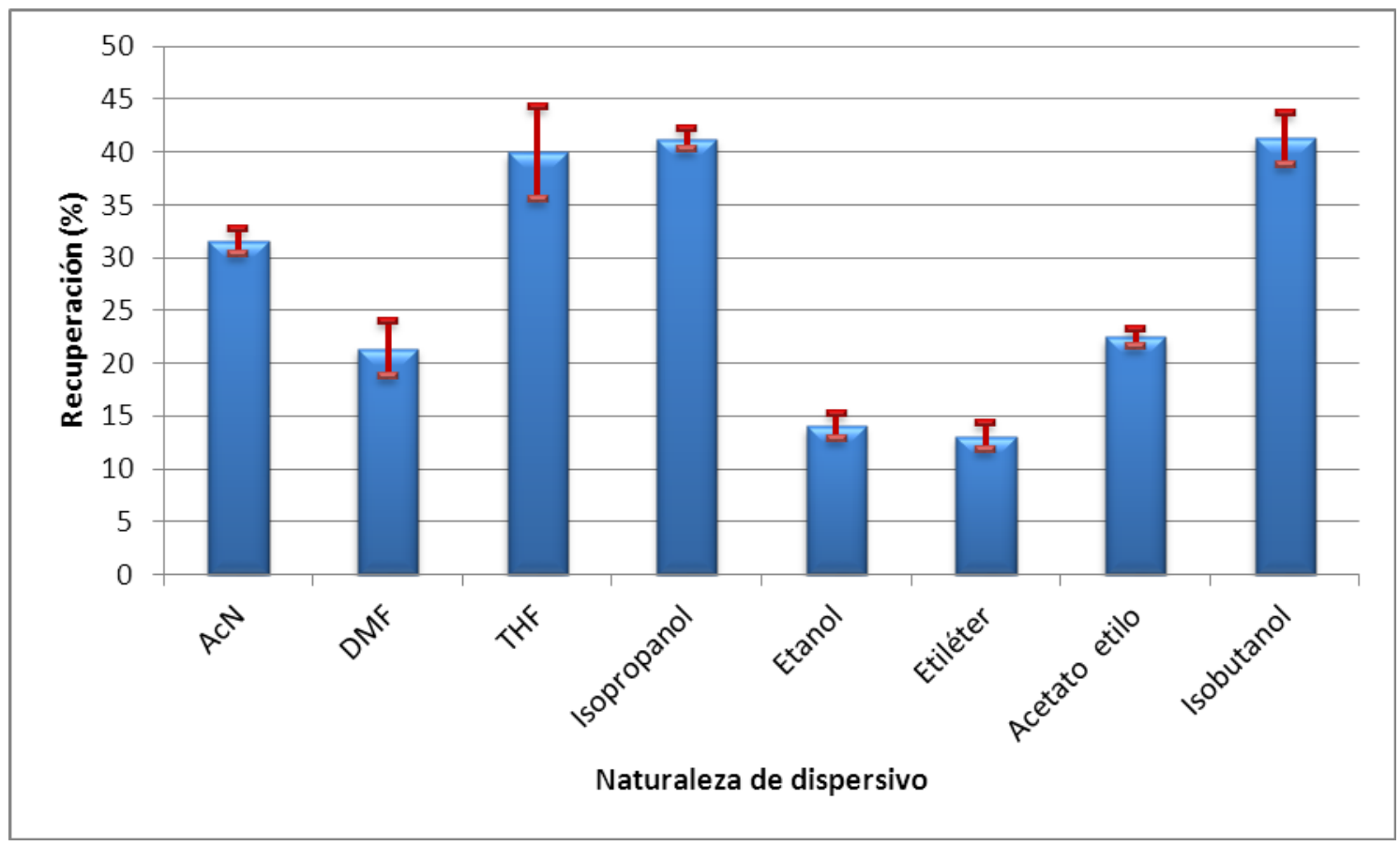

Figura 21. Influencia de la naturaleza del disolvente dispersivo en el porcentaje de recuperación

\subsubsection{OPTIMIZACIÓN DEL VOLUMEN DE DISOLVENTE EXTRACTANTE}

El siguiente paso era estudiar el volumen de disolvente extractante. Para ello, se hizo el estudio en un rango de volúmenes de extractante de 500 a $1500 \mu \mathrm{L}$.

La Figura 22 muestra que para volúmenes de extractante superiores a $1000 \mu \mathrm{L}$, la recuperación conseguida no aumentaba significativamente, manteniéndose prácticamente constante. En base a esto, se decidió tomar como volumen óptimo de extractante $1000 \mu \mathrm{L}$. 


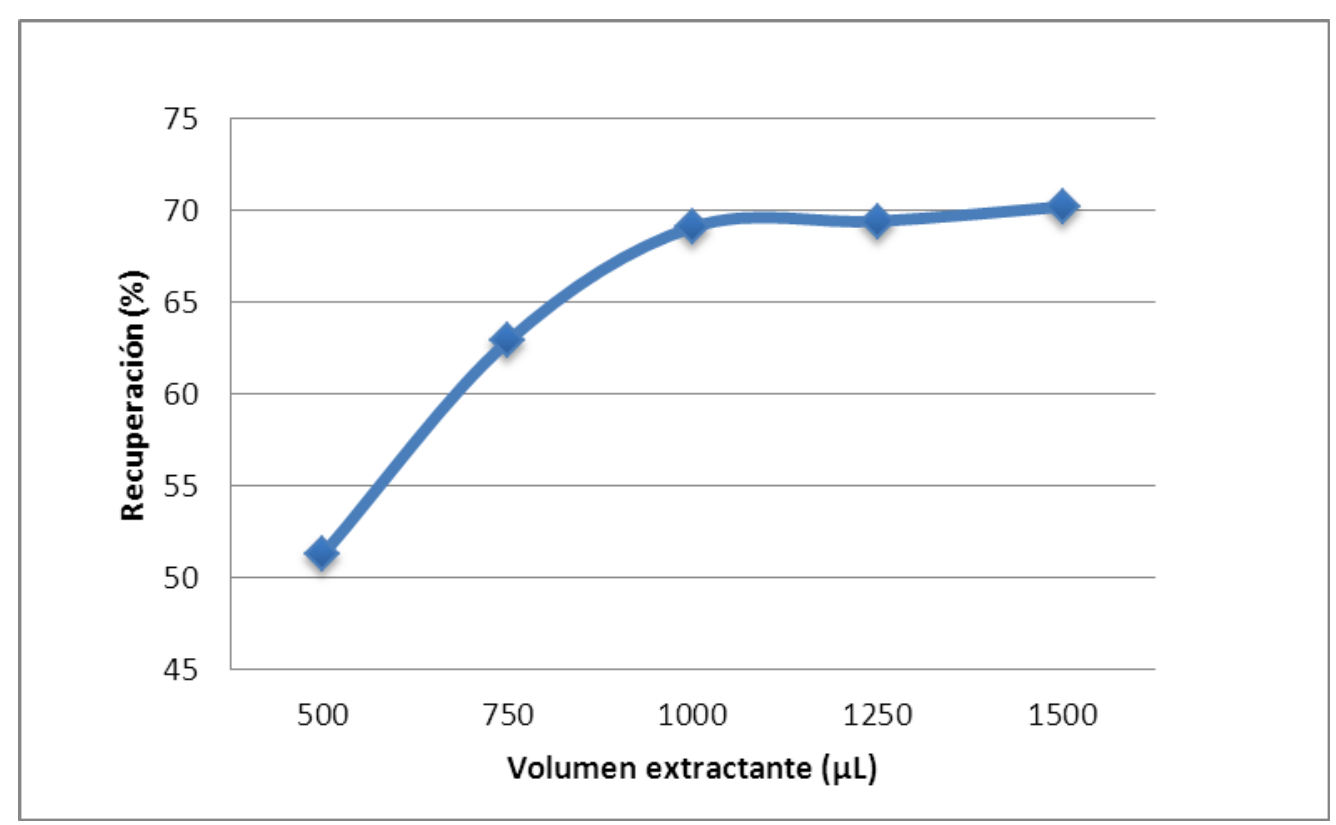

Figura 22. Influencia del volumen de disolvente extractante en el porcentaje de recuperación

\subsubsection{OPTIMIZACIÓN DEL VOLUMEN DE DISOLVENTE DISPERSIVO}

Posteriormente, se efectuó la optimización del volumen de dispersivo, estudiando un rango de volúmenes de 500 a $1500 \mu \mathrm{L}$.

En la Figura 23 se puede observar que los mejores resultados en cuanto a recuperación se obtuvieron para volúmenes de dispersivo de 1000 y $1250 \mu \mathrm{L}$, pues a partir de ahí se observa una disminución en la recuperación. Se selecciona como volumen óptimo $1000 \mu \mathrm{L}$, puesto que la diferencia en cuanto a recuperación, respecto del volumen de $1200 \mu \mathrm{L}$, es insignificante y, así reducimos el consumo de disolvente. 


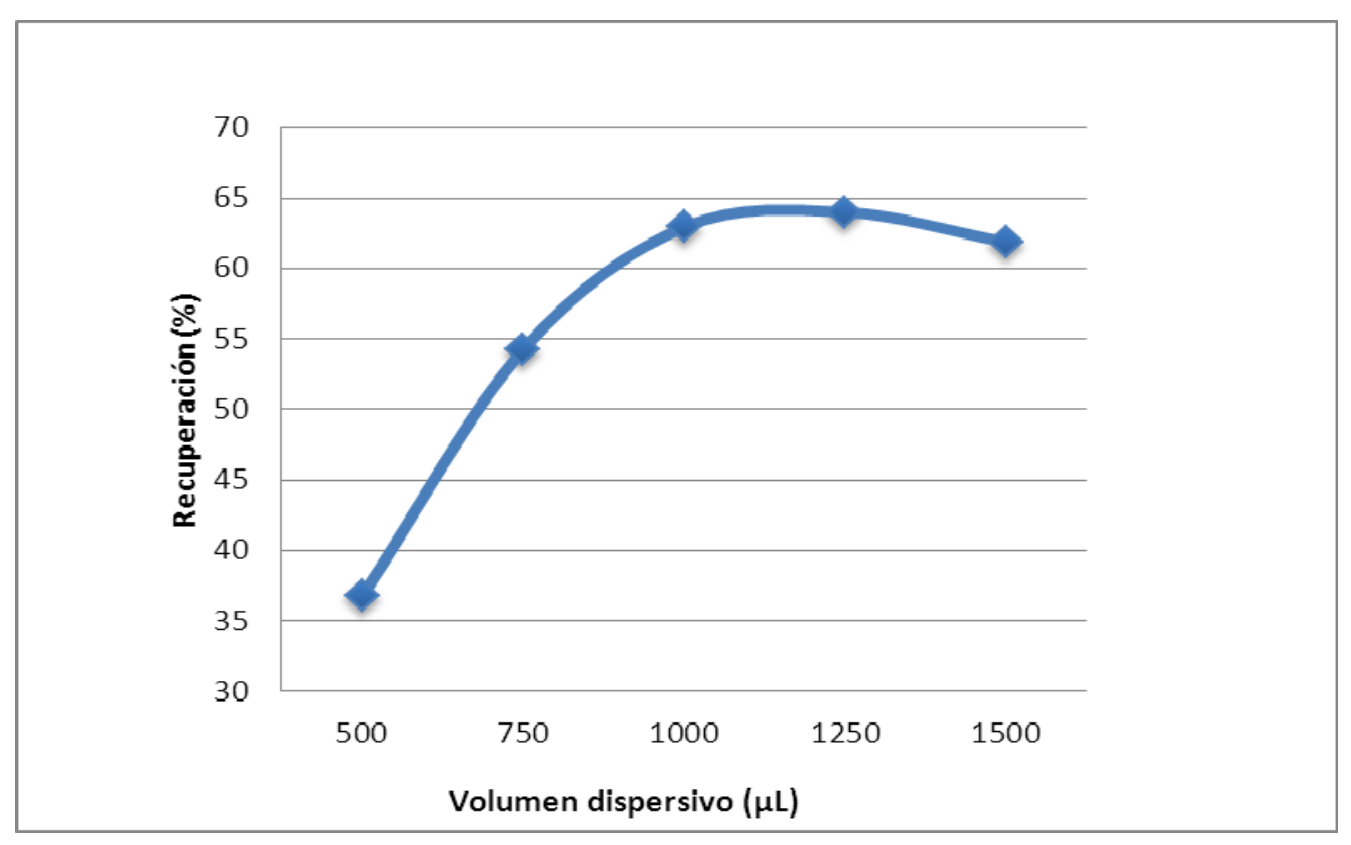

Figura 23. Influencia del volumen de disolvente dispersivo en el porcentaje de recuperación

\subsubsection{OPTIMIZACIÓN DEL PORCENTAJE DE SAL AÑADIDO}

En este apartado, se llevó a cabo la optimización del porcentaje de sal añadida a la muestra. En bibliografía [68-72], la sal más utilizada es $\mathrm{NaCl}$ y los porcentajes utilizados en DLLME suelen variar entre 0 y $10 \%$. Sin embargo, en nuestro caso y como muestra la Figura 24, se obtenían los mejores resultados para porcentajes de $\mathrm{NaCl}$ muy superiores a los mencionados en bibliografía.

Para llevar a cabo esta optimización, se estudió la influencia del porcentaje de sal añadido a la muestra, en el rango de 0 a $30 \%$ ya que el máximo porcentaje de $\mathrm{NaCl}$ que se puede solubilizar en muestras acuosas, a temperatura ambiente, es aprox. el 33\%, aunque en nuestro caso a partir de $30 \%$ observamos que la solubilización total era muy lenta y no se observaba diferencia en el comportamiento.

La Figura 24 muestra que los mejores resultados se obtuvieron para un porcentaje de $\mathrm{NaCl}$ del $20 \%$. 


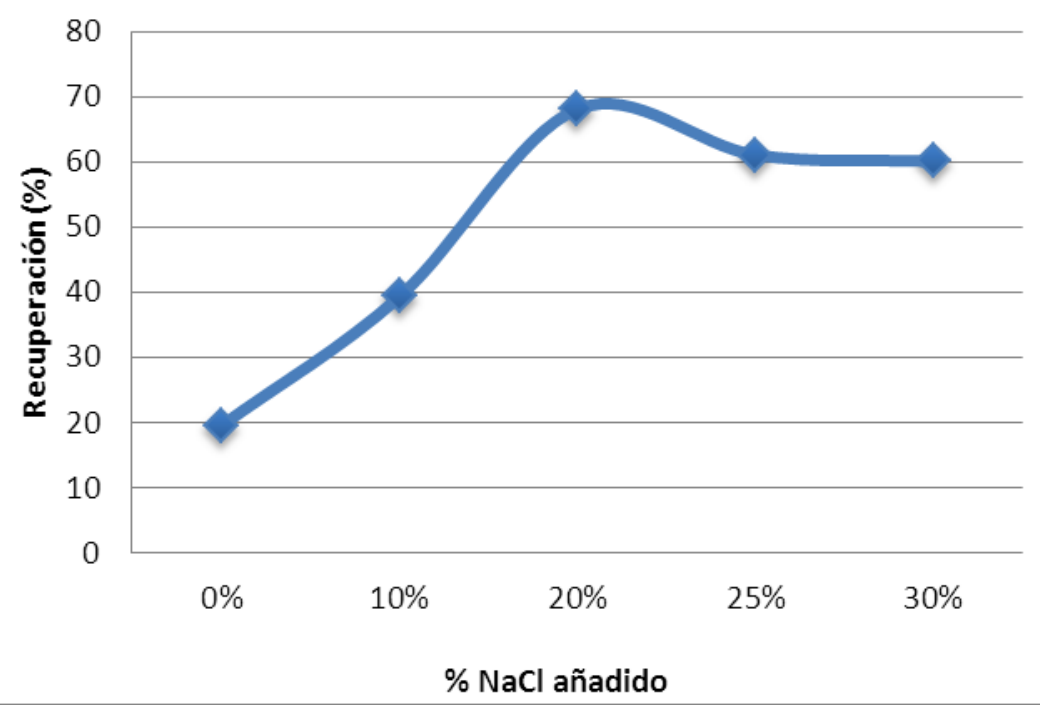

Figura 24. Influencia del porcentaje de $\mathrm{NaCl}$ añadido en el porcentaje de recuperación

\subsubsection{OPTIMIZACIÓN DEL VOLUMEN DE MUESTRA}

Se estudió a continuación el volumen de muestra tratado, seleccionando los siguientes volúmenes: 5,7 y $10 \mathrm{ml}$ de muestra. Tomando los valores óptimos encontrados para los demás parámetros en las experiencias anteriores, los mejores resultados, expresados en términos de porcentaje de recuperación durante la extracción, se obtuvieron para un volumen de muestra de $5 \mathrm{ml}$, como queda demostrado en la Figura 25.

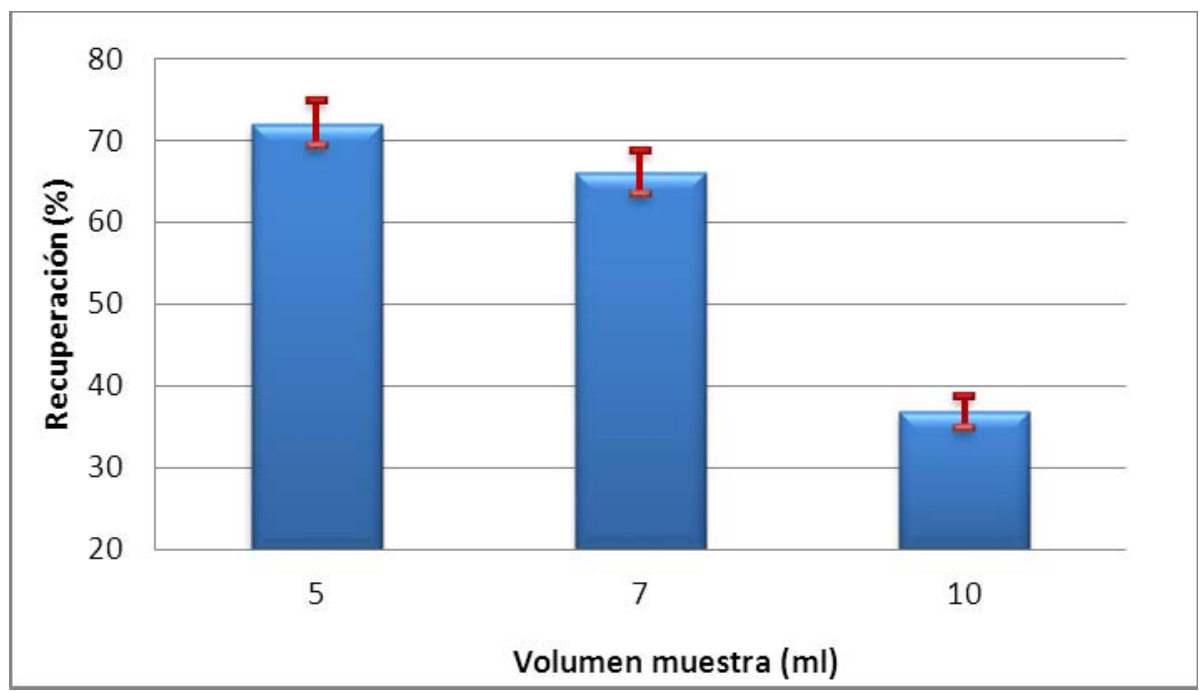

Figura 25 .Influencia del volumen de muestra en el porcentaje de recuperación 


\subsubsection{OPTIMIZACIÓN DEL TIEMPO DE CENTRIFUGACIÓN}

Finalmente se estudió la influencia del tiempo de centrifugación, para una velocidad fija de $5000 \mathrm{rpm}$, en el porcentaje de recuperación. Para ello, se llevó a cabo la optimización de este parámetro en un rango de 2 a 7 minutos. El tiempo óptimo de centrifugación se fijó en 5 minutos, pues era este tiempo el que permitía obtener una mejor eficacia en la extracción.

La Figura 26 muestra la influencia del tiempo de centrifugación en el porcentaje de recuperación del analito presente en la muestra.

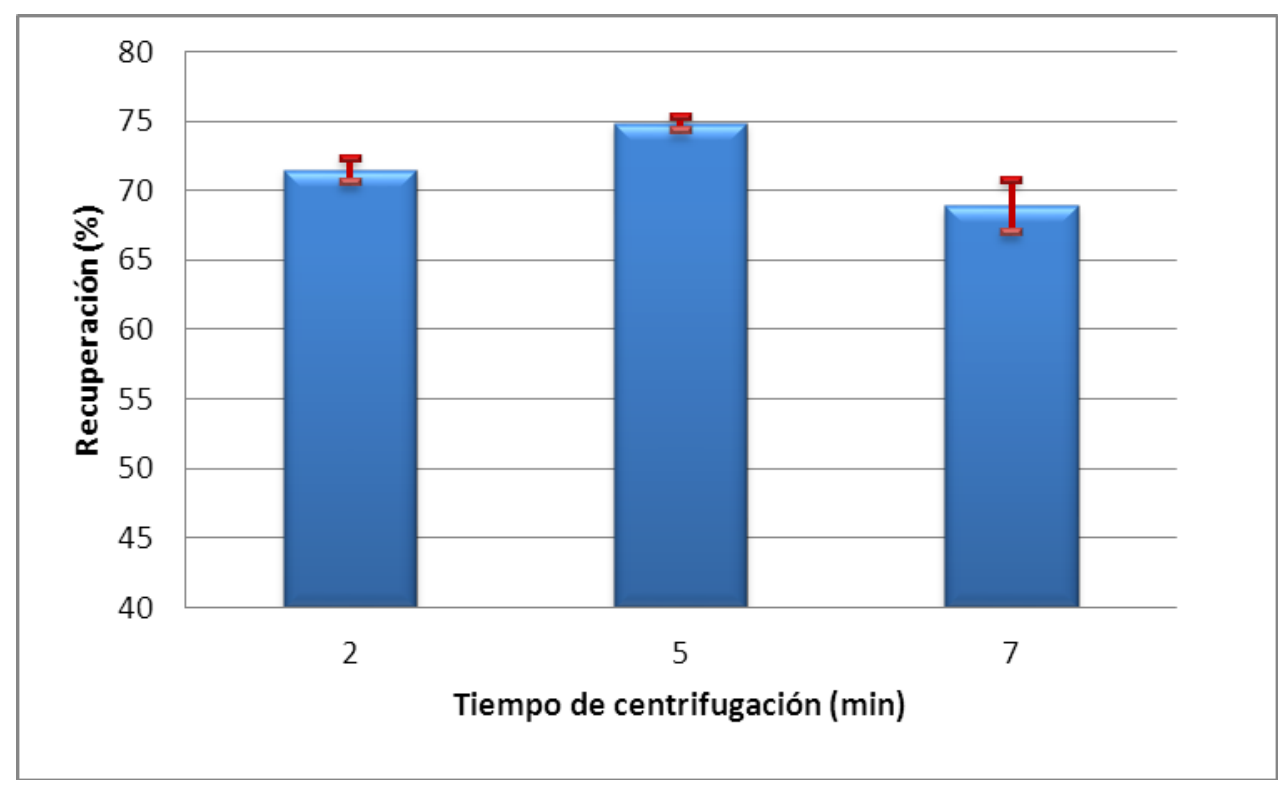

Figura 26. Influencia del tiempo de centrifugación en el porcentaje de recuperación

Una vez optimizadas todas estas variables, para concluir el proceso de DLLME, después de haber centrifugado se recoge la parte orgánica que, como hemos indicado anteriormente, queda en la parte inferior del tubo Falcon y se lleva a sequedad con corriente de $\mathrm{N}_{2}$. Seguidamente reconstituimos, añadiendo $0,5 \mathrm{ml}$ de agua a $\mathrm{pH} 4$ acidificada con ácido acético.

A continuación, se muestra un resumen de la optimización del tratamiento de muestra, indicando los valores seleccionados para cada uno de los factores estudiados (Tabla 8). 
Tabla 8. Resumen de los valores óptimos de las variables que afectan al procedimiento de DLLME

\begin{tabular}{|l|c|}
\hline \multicolumn{1}{|c|}{ Variables } & Condiciones óptimas \\
\hline Naturaleza y volumen disolvente extractante & $1000 \mu \mathrm{L}$ Cloroformo \\
\hline Naturaleza y volumen disolvente dispersante & $1000 \mu \mathrm{L}$ Isopropanol \\
\hline Porcentaje de sal añadido & $20 \%$ \\
\hline Volumen de muestra & $5 \mathrm{ml}$ \\
\hline Volumen recomposición en agua $\mathrm{pH} 4$ & $0,5 \mathrm{ml}$ \\
\hline Parámetros centrifugación & 5 minutos, $5000 \mathrm{rpm}$ \\
\hline
\end{tabular}

\subsection{ESTUDIOS PRELIMINARES EN ZUMOS DE FRUTA COMERCIALES}

Al registrar el electroferograma de muestras de zumos comerciales de manzana, uva, plátano, piña y tomate de diferentes marcas comerciales, nos encontramos con el hecho de que todos ellos presentaban concentraciones variables de HMF, el cual, como hemos indicado anteriormente, aparece durante el procesado de los zumos de fruta, y de patulina, de acuerdo con los datos recogidos en bibliografía $[12,13]$.

En las muestras de zumo comercial, no existía una buena resolución entre la señal del HMF y la de la patulina, de modo que se optó por añadir un modificador orgánico al tampón electroforético.

Generalmente, estos modificadores son disolventes orgánicos que se añaden a la disolución tampón para alterar su polaridad y viscosidad y, como consecuencia de ello, modificar el EOF y la movilidad electroforética de los analitos, influyendo fundamentalmente en el tiempo de migración y la altura de pico de los analitos.

Consultando la bibliografía existente [41], se decidió probar la adición de un 5\% de AcN al tampón electroforético. Puesto que se obtuvieron unos resultados aceptables, no fue necesario probar otros modificadores orgánicos ni otras concentraciones de modificador. 
La presencia del modificador orgánico no influyó significativamente en el área de pico, aunque sí sobre la resolución que aumentó notablemente, incrementándose ligeramente el tiempo de migración de la patulina, tal y como se observa en la Figura 27.

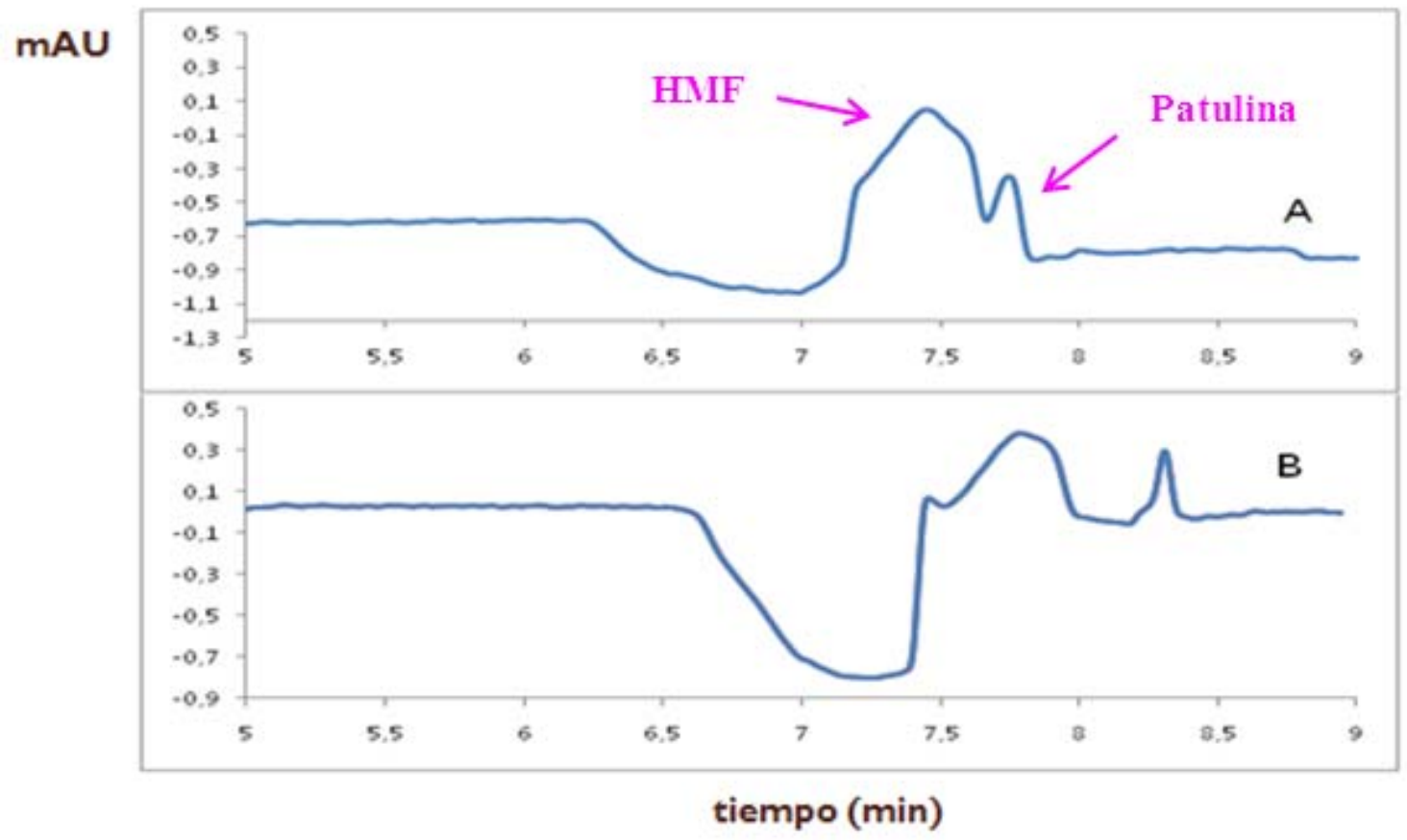

Figura 27. Electroferogramas obtenidos al analizar zumo de manzana comercial: A) sin modificador orgánico en el tampón electroforético, B) con 5\% AcN

Como resultado, el tampón electroforético en los estudios sucesivos consistió en tetraborato sódico $65 \mathrm{mM}$ con SDS $35 \mathrm{mM}$ y 5\% AcN v/v.

Para llevar a cabo la caracterización del método y obtener los parámetros de calidad del mismo en el zumo de fruta, era necesario tener una matriz que estuviera exenta de patulina. Puesto que el análisis de los zumos comerciales analizados indicaba la presencia de patulina, se decidió preparar zumo de manzana en el laboratorio, utilizando para ello una licuadora. De esta forma, tal y como se muestra en la Figura 28, al aplicar el procedimiento de DLLME antes optimizado y llevar a cabo el análisis mediante MEKC se obtuvo una matriz que no presentaba ningún pico al tiempo de migración de la patulina ni, incluso, al tiempo de migración del HMF, pues éste aparece durante la degradación de la fructosa. Solamente se detectó un pico en el extracto a un tiempo superior que no interfería en el análisis de la patulina. 




Figura 28. Electroferogramas obtenidos en el análisis de zumo de manzana natural: A)

Blanco de zumo de manzana, B) Zumo de manzana fortificado con $20 \mu \mathrm{g} \cdot \mathrm{L}^{-1}$ de patulina

\subsection{CARACTERIZACIÓN DEL MÉTODO PROPUESTO}

Para demostrar la validez del método propuesto y su aplicabilidad para el análisis de muestras de zumo de manzana, se siguieron los siguientes pasos:

- Establecimiento de la función de calibrado con muestras de zumo de manzana natural. Estimación de los parámetros estadísticos y los límites de detección y cuantificación.

- Estudio de la precisión del método.

- Estudio de la veracidad del método a través de ensayos de recuperación sobre muestras fortificadas.

A continuación se comentan cada una de estas etapas. 


\subsubsection{ESTABLECIMIENTO DE LA FUNCIÓN DE CALIBRADO, DE LOS PARÁMETROS ESTADÍSTICOS Y DE LOS LÍMITES DE DETECCIÓN Y CUANTIFICACIÓN}

Se estableció la correspondiente función de calibrado en presencia de matriz, usando para ello zumo de manzana recién preparado exento de patulina. La función de calibrado para la patulina se obtuvo mediante fortificación de las muestras, antes del tratamiento, con concentraciones crecientes. La linealidad de la respuesta se estableció a partir de cinco niveles de concentración correspondientes a $5 ; 20 ; 50 ; 75$ y $100 \mu 1 \cdot \mathrm{L}^{-1} \mathrm{de}$ patulina en la muestra (que se correspondería con 50; 200; 500; 750 y $1000 \mu 1 \cdot \mathrm{L}^{-1}$ en el extracto final ya que con la DLLME se obtiene un factor de preconcentración de 10). Se prepararon dos muestras para cada nivel de concentración (réplicas experimentales), y cada una fue inyectada por triplicado (réplicas instrumentales). La función de calibrado se estableció considerando las áreas de pico en función de la concentración.

Su representación gráfica se muestra en la Figura 29.

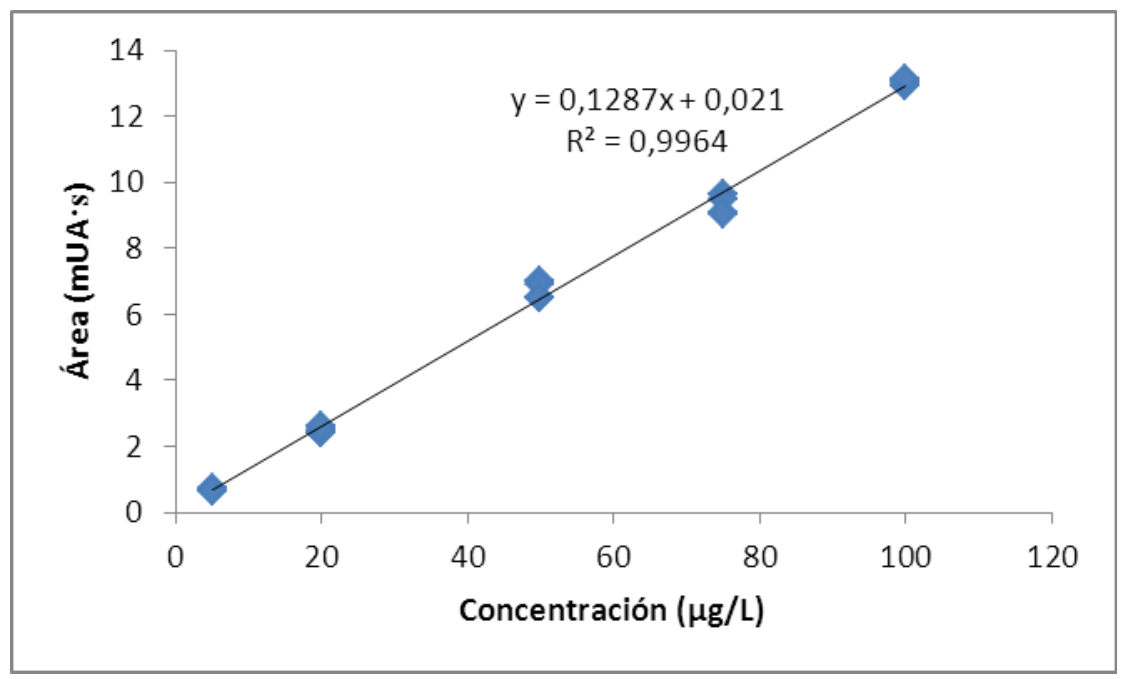

Figura 29. Recta de calibrado de la patulina para la muestra de zumo de manzana

Los parámetros estadísticos fueron calculados por medio de la regresión lineal por mínimos cuadrados. El coeficiente de determinación obtenido $\left(\mathrm{R}^{2}=0,9964\right)$ confirma una linealidad satisfactoria en el rango estudiado. El límite de detección (LOD) fue $0,71 \mu \mathrm{g} \cdot \mathrm{L}^{-1}$ y el de cuantificación (LOQ) $2,4 \mu \mathrm{g} \cdot \mathrm{L}^{-1}$, y se calcularon a partir de la relación $\mathrm{S} / \mathrm{N}$ multiplicando por 3 y 10, respectivamente. 
Como puede observarse, el rango dinámico lineal es extenso (de $2,1 \mu 1 \cdot \mathrm{L}^{-1}$ a 100 $\mu 1 \cdot \mathrm{L}^{-1}$ ) y los valores obtenidos para el límite de detección y cuantificación están muy por debajo de los contenidos máximos permitidos por legislación $\left(50 \mu \mathrm{g} \cdot \mathrm{L}^{-1}\right.$ en zumo de manzana y $10 \mu \mathrm{g} \cdot \mathrm{L}^{-1}$ en alimentos a base de manzana para lactantes y niños de corta edad).

\subsubsection{ESTUDIO DE LA PRECISIÓN}

La precisión del método completo se evaluó en términos de repetitividad y precisión intermedia. La repetitividad fue evaluada en el mismo día por aplicación del método propuesto a tres muestras (réplicas experimentales) para cada nivel de concentración de patulina (Nivel 1: $20 \mu \mathrm{g} \mathrm{L}^{-1}$, Nivel 2: $50 \mu \mathrm{g} \mathrm{L}^{-1}$, Nivel 3: $75 \mu \mathrm{g} \mathrm{L}^{-1}$ ), y cada una fue inyectada por triplicado (réplicas instrumentales). La precisión intermedia se evaluó durante cinco días consecutivos con un procedimiento similar al del estudio de repetitividad. Los resultados expresados como desviaciones estándar relativas (DER) de las áreas de pico se muestran en la Tabla 9. Como se puede comprobar, la precisión fue aceptable en todos los casos.

Tabla 9. Estudio de precisión para el método propuesto

\begin{tabular}{lcc}
\hline & Repetitividad (*n=9) & Precisión intermedia (*n=15) \\
\hline Nivel $1\left(\mathbf{2 0} \boldsymbol{\mu} \mathbf{g ~ L}^{-1}\right)$ & 8,5 & 9,6 \\
Nivel 2 $\left(\mathbf{5 0} \boldsymbol{\mu} \mathbf{g ~ L}^{-1}\right)$ & 2,6 & 4,9 \\
Nivel 3 $\left(\mathbf{7 5} \boldsymbol{\mu} \mathbf{g ~ L}^{-1}\right)$ & 3,1 & 6,4 \\
\hline
\end{tabular}

(*n: $n^{o}$ de réplicas totales)

\subsubsection{ESTUDIO DE LA VERACIDAD DEL MÉTODO PROPUESTO}

Para comprobar la veracidad del método propuesto para muestras de zumo de manzana se llevaron a cabo ensayos de recuperación, añadiendo concentraciones crecientes de patulina a muestras zumo de manzana, exentas de la misma. Las muestras de zumo de manzana se fortificaron con patulina a diferentes niveles de concentración 
(Nivel 0: $5 \mu \mathrm{g} \mathrm{L}^{-1}$, Nivel 1: $20 \mu \mathrm{g} \mathrm{L}^{-1}$, Nivel 2: $50 \mu \mathrm{g} \mathrm{L}^{-1}$, Nivel 3: $75 \mu \mathrm{g} \mathrm{L}^{-1}$ ). Cada nivel de concentración se preparó por triplicado y se inyectó tres veces. Las recuperaciones absolutas fueron calculadas comparando las áreas de pico de las muestras de zumo fortificadas antes del procedimiento DLLME con las áreas de pico de muestras de zumo fortificadas después del procedimiento DLLME. Todas las recuperaciones obtenidas fueron superiores al 75\%, con DER aceptables en todos los casos, cumpliendo con los criterios establecidos en la legislación [16]. Los resultados de estos estudios se muestran en la Tabla 10.

Tabla 10. Recuperaciones obtenidas en muestras de zumo de manzana fortificadas a distintos niveles de concentración

\begin{tabular}{|c|c|c|}
\hline & & Zumo de manzana \\
\hline \multirow{2}{*}{ Nivel $0\left(5 \mu g \mathrm{~L}^{-1}\right)$} & R (\%) & 80,0 \\
\hline & $\operatorname{DER}(\%)$ & 4,7 \\
\hline \multirow{2}{*}{ Nivel $1\left(20 \mu \mathrm{g} \mathrm{L}^{-1}\right)$} & R (\%) & 75,6 \\
\hline & $\operatorname{DER}(\%)$ & 8,5 \\
\hline \multirow{2}{*}{ Nivel $2\left(50 \mu \mathrm{g} \mathrm{L}^{-1}\right)$} & R (\%) & 75,2 \\
\hline & $\operatorname{DER}(\%)$ & 2,6 \\
\hline \multirow{2}{*}{ Nivel $3\left(75 \mu \mathrm{g} \mathrm{L}^{-1}\right)$} & R (\%) & 76,1 \\
\hline & DER (\%) & 3,1 \\
\hline
\end{tabular}

$(n=6)$

En la Figura 30 se muestran los electroferogramas obtenidos al inyectar muestras de zumo de manzana a las concentraciones consideradas en el ensayo de recuperaciones. 


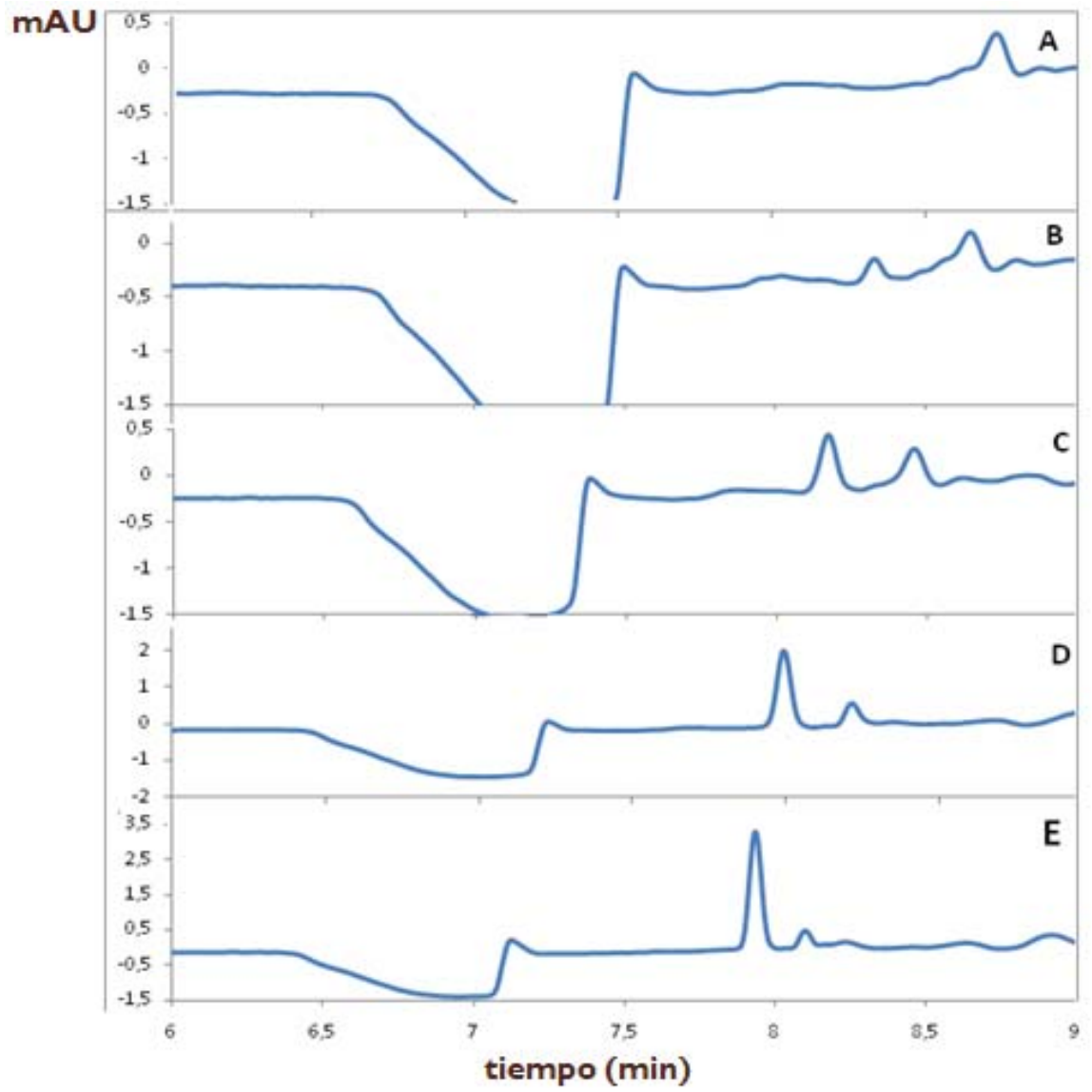

Figura 30. Electroferogramas de muestras de zumo de manzana: (A) Blanco de muestra y Muestras fortificadas con (B) $5 \mu \mathrm{g} \cdot \mathrm{L}^{-1}$ de patulina (C) $20 \mu \mathrm{g} \cdot \mathrm{L}^{-1}$ de patulina (D) 50 $\mu \mathrm{g} \cdot \mathrm{L}^{-1}$ de patulina (E) $75 \mu \mathrm{g} \cdot \mathrm{L}^{-1}$ de patulina 


\section{VENTAJAS DE LA METODOLOGÍA PROPUESTA}

En esta Memoria se propone el empleo de MEKC-UV-Vis como técnica de análisis para la determinación de patulina en zumos de manzana. Considerando las características del método empleado frente al método cromatográfico que utiliza el mismo sistema de detección, podemos destacar que el método usando MEKC presenta como ventaja un menor consumo de disolventes y es más simple y económico que el basado en el empleo de HPLC [13], como muestra la Tabla 11.

Tabla 11. Comparación del método empleado con el método basado en HPLC, ambos con detección UV-Vis.

\begin{tabular}{|l|c|c|}
\hline \multicolumn{1}{|c|}{ Criterios } & HPLC & MEKC \\
\hline Tiempo de análisis & $7.8 \mathrm{~min}$ & $9.0 \mathrm{~min}$ \\
\hline Recuperación (\%) & $78.0 \%$ & $75.2-80.0 \%$ \\
RSD $(\%)$ & $7.8 \%$ & $3.1-8.5 \%$ \\
\hline LOD $\left(\mu \mathrm{g} \cdot \mathrm{L}^{-1}\right)$ & 0.60 & 0.71 \\
LOQ $\left(\mu \mathrm{g} \cdot \mathrm{L}^{-1}\right)$ & 1.6 & 2.4 \\
\hline Volumen muestra & microlitros & nanolitros \\
\hline Funcionamiento & Complejo & Sencillo \\
\hline Equipamiento & & Simple \\
& Sofisticado (elevadas presiones) & Barato \\
\hline Consumo disolventes & Caro & Bajo \\
\hline Reproducibilidad & Elevado & Comparable \\
\hline
\end{tabular}

En cuanto al tratamiento de muestra desarrollado, en la Tabla 12 se ha realizado una comparación del método mediante DLLME optimizado en este trabajo con los métodos usualmente empleados en el análisis de patulina en zumos de frutas. Como puede observarse, nuestro método requiere una cantidad de disolventes muy inferior a los basados en SPE o LLE [40,51], muestra una precisión satisfactoria y superior a LLE y el número de muestras procesadas por hora es muy superior a ambos, siendo además muy simple en su manipulación y el de menor impacto ambiental. Por ello, la combinación MEKC-DLLME se plantea como un método muy recomendable en 
análisis de rutina para laboratorios que realicen control de calidad y seguridad alimentaria.

Tabla 12. Comparación de los tratamientos de muestra más utilizados en el análisis de patulina con el método de DLLME propuesto

\begin{tabular}{|l|c|c|c|}
\hline \multicolumn{1}{|c|}{ Criterios } & SPE & LLE & DLLME \\
\hline Cantidad muestra & $1 \mathrm{~g}$ & $5 \mathrm{~g}$ & $5 \mathrm{ml}$ \\
\hline Cantidad disolventes & $30 \mathrm{ml}$ (elevada) & $100 \mathrm{ml}$ (muy elevada) & $2 \mathrm{ml}$ (muy baja) \\
\hline Recuperación (\%) & $96.4-114.1$ & $75.2-89.2$ & $75.2-80.0$ \\
\hline CV (\%) & $1.62-4.82$ & $6.37-9.46$ & $3.1-8.5$ \\
\hline Funcionamiento & De larga duración & De larga duración & $\begin{array}{c}\text { Simple } \\
\text { Rápido }\end{array}$ \\
\hline Muestras tratadas en 1 hora & 2 & 1 & 10 \\
\hline Impacto medioambiental & Moderado & Alto & Muy bajo \\
\hline
\end{tabular}




\section{APLICACIONES}

Una vez planteada y demostrada por primera vez la validez de la DLLME como técnica de extracción de patulina en zumo de manzana, previa al análisis mediante MEKC, se ha llevado a cabo la aplicación de esta metodología a un estudio de muestras comerciales de zumos de manzana infantiles, con objeto de evaluar si cumplen con la legislación en cuanto a los contenidos máximos establecidos $\left(10 \mu \mathrm{g} \cdot \mathrm{L}^{-1}\right)$.

En este estudio se han analizado zumos de manzana destinados a consumo infantil obtenidos en comercios locales, de marcas y lotes diferentes, utilizando la metodología de calibrado por adición de patrón, aplicando el procedimiento analítico propuesto. Para cada zumo, se tomaron 6 muestras de $5 \mathrm{ml}$ cada una, a las que se les adicionaron concentraciones crecientes de patulina $\left(0,5,20,50,75\right.$ y $\left.100 \mu \mathrm{g} \cdot \mathrm{L}^{-1}\right)$. Seguidamente se les aplicó el procedimiento de tratamiento de muestra propuesto mediante DLLME y se llevó a cabo el análisis usando MEKC.

A modo de ejemplo, la Figura 31 muestra el calibrado por adición de patrón obtenido para uno de estos zumos comerciales (en concreto el de la Marca A y lote 1).

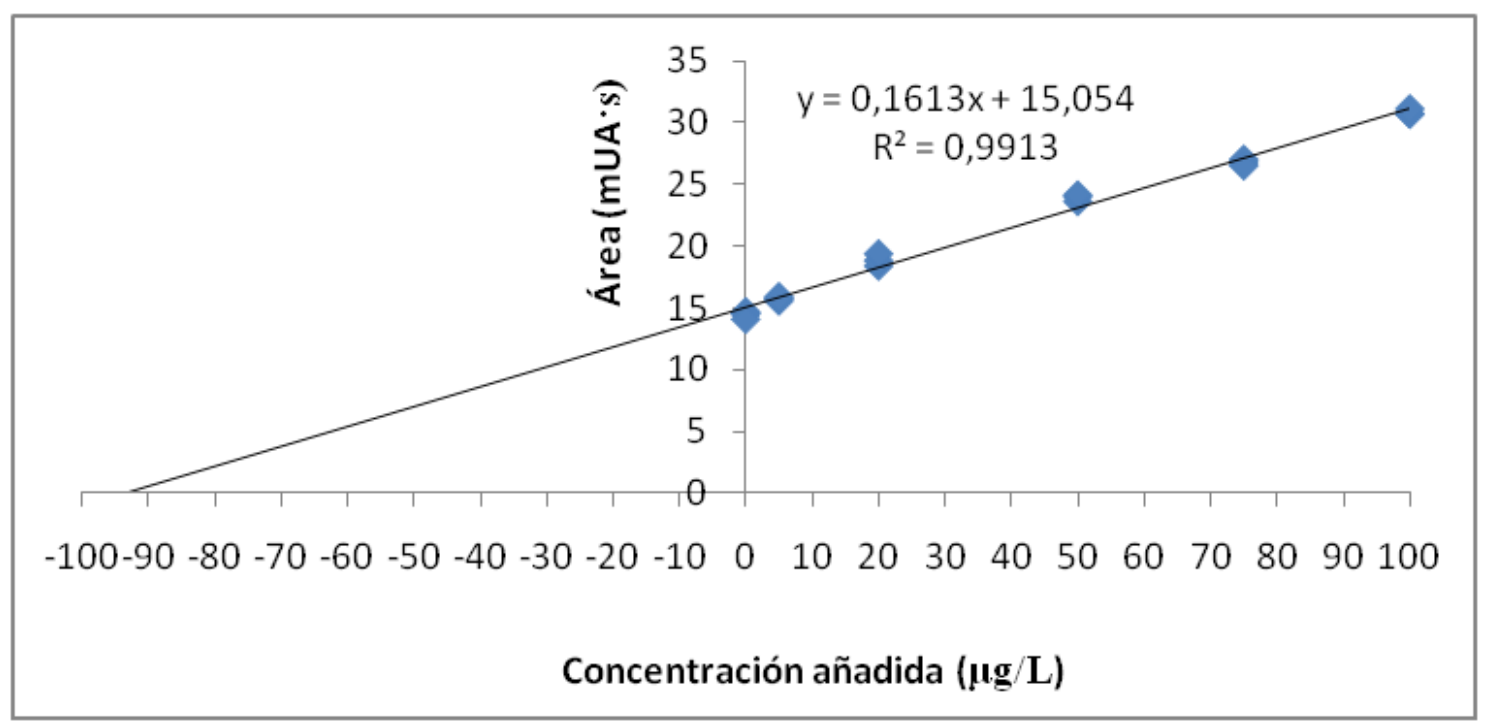

Figura 31. Calibrado por adición de patrón del zumo de la Marca A y lote 1

Como resultado de la aplicación de la metodología mencionada, se observó que la muestra del zumo de manzana de la Marca A y lote 1, tenía una concentración de patulina de $93 \mu \mathrm{g} \cdot \mathrm{L}^{-1}$. 
La Tabla 13 muestra los resultados obtenidos para los zumos analizados.

Tabla 13. Resultados en zumos de manzana infantiles analizados

\begin{tabular}{|c|c|c|}
\hline Muestra & Concentración $\left(\boldsymbol{\mu g} \cdot \mathrm{L}^{\mathbf{- 1}}\right)$ & Apto para consumo $\left(<\mathbf{1 0} \boldsymbol{\mu g} \cdot \mathbf{L}^{\mathbf{- 1}}\right)$ \\
\hline Marca A, lote 1 & 93 & NO \\
\hline Marca A, lote 2 & 84 & NO \\
\hline Marca B, lote 1 & 12 & NO \\
\hline Marca B, lote 2 & 9 & Sí \\
\hline
\end{tabular}




\section{CONCLUSIONES}

En esta Memoria se ha desarrollado una nueva metodología para la extracción de patulina en zumos de manzana, empleando la microextracción líquido-líquido dispersiva (DLLME). Se han optimizado todas las variables que influyen en la eficacia del proceso de extracción, demostrándose por primera vez la utilidad de esta metodología para la extracción de este contaminante en zumos.

Los análisis se han llevado a cabo mediante la aplicación de la cromatografía capilar electrocinética micelar (MEKC) con detección UV-VIS, estudiándose las variables implicadas en el proceso separativo para confirmar los óptimos.

La separación entre patulina y HMF, su principal interferente en zumos, se ha llevado a cabo en un tiempo inferior a 9 minutos.

El método DLLME-MEKC ha sido caracterizado en cuanto a sus parámetros de calidad y validado para el análisis de muestras de zumo de manzana, obteniéndose unos límites de detección y cuantificación muy por debajo de los valores establecidos por la legislación como contenidos máximos de patulina en alimentos.

La precisión del método (tanto intradía como interdía), es aceptable en todos los casos de acuerdo con la legislación establecida.

Los ensayos de recuperación realizados demuestran que el método propuesto cumple con los requisitos, relacionados tanto con porcentajes de recuperación como DER, establecidos por la legislación para los métodos de determinación de patulina en alimentos.

Su aplicación a muestras de zumo de manzana destinado a consumo infantil se ha llevado a cabo utilizando la metodología de adición de patrón, encontrándose en los zumos analizados concentraciones superiores a las $10 \mu \mathrm{g} \cdot \mathrm{L}^{-1}$, establecidas por la legislación.

El método es rápido, simple y robusto al combinar las ventajas de la DLLME (simple, rápida, de gran eficiencia en la extracción, posibilidad de altos factores de preconcetración y bajo consumo de disolventes orgánicos) con las ventajas de la electroforesis capilar (técnica miniaturizada, económica, con cortos tiempo de análisis y gran eficacia). Por todo ello puede considerarse como una estrategia dentro de la Química Analítica Verde, al plantear un método de bajo impacto ambiental. 
Los resultados de este trabajo serán comunicados en modo de cartel en las $13^{a s}$ Jornadas de Análisis Instrumental, a celebrar en Barcelona del 14-16 de Noviembre. 


\section{GLOSARIO}

$\mathrm{AcN}$ - Acetonitrilo

APCI - Ionización química a presión atmosférica

APPI - Fotoionización a presión atmosférica

ASE - Extracción acelerada por disolventes

AOAC - Asociación Oficial de Químicos Analíticos

$\mathrm{CE}$ - Electroforesis capilar

CEC - Electrocromatografía capilar

CEN - Comité Europeo de Normalización

CGE - Electroforesis capilar en gel

CIEF - Isoelectroenfoque capilar

CITP - Isotacoforesis capilar

$\mathrm{CL}$ - Quimioluminiscencia

CMC - Concentración micelar crítica

CZE - Electroforesis capilar zonal

DAD - Detector de diodos en fila

DER - Desviación estándar relativa

DMF - Dimetilformamida

DLLME - Microextracción líquido-líquido dispersiva

EI - Ionización por electrospray

EOF - Flujo electroosmótico

FDA - Administración Federal de Drogas y Alimentos

FL - Fluorescencia

HMF - Hidroximetilfurfural

HPLC - Cromatografía líquida de alta resolución

IAC - Columnas de inmunoafinidad

IARC - Agencia Internacional de Investigación sobre el Cáncer

IDT - Ingesta diaria tolerable

IDTMP - Ingesta diaria tolerable máxima provisional

ISO - Organización Internacional para la Estandarización

ISTP - Ingesta semanal tolerable provisional

JECFA - Comité Mixto FAO/OMS de Expertos en Aditivos Alimentarios

LC - Cromatografía líquida 
LOD - Límite de detección

LOQ - Límite de cuantificación

LPME - Microextracción líquido-líquido

MAE - Extracción asistida por microondas

MEKC - Cromatografía capilar electrocinética micelar

MS - Espectrometría de masas

MSPD - Dispersión de matriz en fase sólida

NOAEL - Nivel de efecto adverso no observado

SDS - Dodecil sulfato sódico

SFE - Extracción por fluidos supercríticos

SPE - Extracción en fase sólida

SPME - Microextracción en fase sólida

THF - Tetrahidrofurano

TLC - Cromatografía en capa fina

Tris - Tris(hidroximetil)aminometano

UE - Unión Europea

$\mathrm{UV} / \mathrm{Vis}$ - Ultravioleta/visible 


\section{REFERENCIAS}

[1] Reglamento (CE) $\mathrm{N}^{\mathrm{o}} 1881 / 2006,19$ diciembre 2006, por el que se fija el contenido máximo de determinados contaminantes en los productos alimenticios.

[2] González, L.; Soriano, J. M. (2007). Introducción. En: Micotoxinas en alimentos. Editor: José Miguel Soriano del Castillo et al. Ediciones Díaz de Santos. Cap. 1, págs. 3-4.

[3] González, L.; Soriano, J. M. (2007). Toxicidad y evaluación de riesgos. En: Micotoxinas en alimentos. Editor: José Miguel Soriano del Castillo et al. Ediciones Díaz de Santos. Cap. 2, págs. 15-19.

[4] International Agency for Research on Cancer (IARC) (2010). Disponible en: http://www.iarc.fr. Última visita realizada: 26-07-2011.

[5] González, L.; Soriano, J. M. (2007). Patulina. En: Micotoxinas en alimentos. Editor: José Miguel Soriano del Castillo et al. Ediciones Díaz de Santos. Cap. 12, págs. 239-249.

[6] Conseil Supérieur D’Hygiène Publique de France. (1999). Les mycotoxines dans l'alimentation; evaluation et gestion du risqué. Editions Lavoisier, Tec \& Doc., págs. 279-293.

[7] Ministry of Agriculture, Fisheries and Food. MAFF (1993). London. Mycotoxins; third report. In food surveillance paper Mo., 36, págs. 46-50.

[8] World Health Organization (1995). 44th Report of the Joint FAO/WHO Expert Committee on Food Additives, Tech. Report Series, 859, Geneva, Switzerland.

[9] Scientific Committee on Food, 1996: Reports of the SCF (Thirty-fifth series), 1996.

[10] World Health Organization (1990). Report of the Joint FAO/WHO Expert Committee on Food Additives, Tech. Report Series, 789 Geneva, Switzerland.

[11] World Health Organization (1995). $44^{\text {th }}$ Report of the Joint FAO/WHO Expert Committee on Food Additives, Tech. Report Series, 859 Geneva, Switzerland.

[12] Gaspar, E.; Lucena, A. (2009). Improved HPLC methodology for food control - furfurals and patulin as markers of quality. Food Chemistry, 114, págs. 1576-1582.

[13] Murillo-Arbizu, M., González-Peñas, E., Amézqueta, S. (2010). Comparison between capillary electrophoresis and high performance liquid chromatography for the study of the occurrence of patulin in Apple juice intended for infants. Food and Chemical Toxicology, 48, págs. 2429-2434.

[14] Park, D. L.; Njapau, H.; Coker, R. D. (1998). Sampling programs for mycotoxins: Perspectives and recommendations. En: Miraglia, M., van Edmond, H. P., Brera, C., Gilbert, J. (eds.). Mycotoxins and phycotoxins-developments in chemistry, toxicology and food safety. Alaken, Inc. Colorado, EEUU. Págs. 53-64.

[15] Comité Europeo de Normalización. (1999). Food analysis- Biotoxins-criteria of analytical method of mycotoxins. CEN Report CR 13505. Bruselas, Bélgica.

[16] Reglamento 401/2006 (CE), de 23 de Febrero de 2006, por el que se establecen los métodos de muestreo y de análisis para el control oficial del contenido de micotoxinas en los productos alimenticios.

[17] Official Methods of Analysis of AOAC International. 17th Edition (2002). W. Horwitz (ed). AOAC International. Gaithersburg, MD, EEUU. 
[18] Key, P. E.; Patey, A. L., Rowling, S., Wilbourne, S.; Worner, F. W. (1997). International proficiency testing of analytical laboratories from 1990 to 1996: the experiences of the United Kingdom Food Analysis Performance Assessment Scheme. J. AOAC Int., 80, págs. 895-899.

[19] Thompson, M.; Wood, R. (1993). International harmonized protocol for proficiency testing of chemical analytical laboratories. J. AOAC Int., 76, págs. 926-940.

[20] Williams, A. C.; Jorgensen, K.; Donnelly, C., Boenke, A. (1998). The second interlaboratory study of methods for the analysis of ochratoxin A in pig kidney. Report EUR 18458 EN. Office for Official Publications of European Communities. Luxemburgo.

[21] Soriano, J. M.; Moltó, J. C.; Mañes, J. (2007). Análisis de micotoxinas en alimentos. En: Soriano, J. M. (eds.) Micotoxinas en alimentos. Díaz Santos. Madrid, España. Págs. 91-117.

[22] Trucksess, M. W.; Pohland, A. E. (2002). Methods and method evaluation for mycotoxins. Molecular Biotechnology, 22, págs. 287-292.

[23] Turner, N. W.; Subrahmanyam, S.; Piletsky, S. A. (2009). Analytical methods for determination of mycotoxins: A review. Anal. Chim. Acta, 632, págs. 168-180.

[24] Shephard, G. S. (2008). Determination of mycotoxins in human foods. Chem. Soc. Rev., 37, pags. $2468-2477$.

[25] Köppen, R.; Koch, M.; Siegel, D.; Merkel, S.; Maul, R.; Nehls, I. (2010). Determination of mycotoxins in foods: Current state of analytical methods and limitations. Apple Microbiol. Biotechnol., 86, págs. 1595-1612.

[26] Zöllner, P.; Mayer-Helm, B. (2006). Trace mycotoxin analysis in complex biological and food matrices by liquid chromatography-atmospheric pressure ionisation mass spectrometry. J. Chromatogr. A, 1136, págs. 123-169.

[27] Association of Official Analytical Chemists (AOAC), 1984. En: Williams S. (Ed.), Official Methods of Analysis. Virginia, EEUU, pags. 496-497.

[28] Sheu, F.; Shyu, Y.T. (1999). Analysis of patulin in apple juice by diphasic dialysis extraction with in situ acylation and mass spectrometry, J. Agric. Food Chem., 47, págs. 2711-2714.

[29] Koach, J.A.G.; White, K.D.; Trucksess, M.W.; Thomas, F.S. (2000). Capillary gas chromatography/mass spectrometry with chemical ionization and negative ion detection for confirmation of identity of patulin in apple juice. J. AOAC Int., 83, págs. 104-112.

[30] MacDonald, S.; Long, M.; Gilbert, J.; Felqueiras, I. (2000). Liquid chromatography method for determination of patulin in clear and cloudy Apple juices and puree: collaborative study. J. AOAC Int., 83, págs. 1387-1394.

[31] Rupp, H.S.; Turnipseed, S.B. (2000). Confirmation of patulin and 5-hydroxymethylfurfural in apple juice by gas chromatography/mass spectrometry. J. AOAC Int., 83, págs. 612-620.

[32] S.C. Cunha; M.A. Faria; J.O. Fernandes. (2009). Determination of patulin in apple and quince products by GC-MS using ${ }^{13} \mathrm{C}_{5-7}$ patulin as internal standard, Food Chem., 115, págs. 352-359.

[33] Shepard, G.S.; Leggott, N.L. (2000). Chromatographic determination of the mycotoxin patulin in fruit and fruit juices. J. Chromatogr. A, 882, págs. 17-22. 
[34] Gökmen, V.; Acar, J. (1999). Simultaneous determination of 5-hydroxymethylfurfural and patulin in apple juice by liquid chromatography. J. Chromatogr. A, 847, págs. 69-74.

[35] Katerere, D.R.; Stockenstrom, S.; Shepard, G.S. (2008). HPLC-DAD method for the determination of patulin in dried apple rings. Food Control, 19, págs. 389-392.

[36] Sewram, V.; Nair, J. J.; Nieuwoudt, T. W.; Leggott, N. L.; Shephard, G. S. (2000). Determination of patulin in apple juice by high-performance liquid chromatography-atmospheric pressure chemical ionization mass spectrometry. J. Chromatogr. A, 897, págs. 365-374.

[37] Takino, M.; Daishima, S.; Nakahara, T. (2003). Liquid chromatography/mass spectrometric determination of patulin in apple juice using atmospheric pressure photoionization. Rapid Comm. Mass Spectr., 17, págs. 1965-1972.

[38] Vallejo-Córdoba, B.; Vargas-Martínez, G. (2008). Capillary Electrophoresis Aplications for Food Analysis. Handbook of Capillary and Microchip Electrophoresis and Associated Microtechiniques, Third Edition, págs. 853-912.

[39] Tsao, R.; Zhou, T. (2000). Micellar electrokinetic capillary electrophoresis for rapid analysis of patulin in apple cider. J. Agric. Food Chem., 48, págs. 5231-5235.

[40] Murillo-Arbizu, M.; González-Peñas, E.; Hansen, S. H.; Amézqueta, S.; Ostergaard, J. (2008). Development and validation of a microemulsion electrokinetic chromatography method for patulin quantification in commercial apple juice. Food Chem. Toxicol., 46, págs. 2251-2257.

[41] Murillo-Arbizu, M.; González-Peñas, E.; Amézqueta, S. (2008). Determination of patulin in commercial apple juice by micellar electrokinetic chromatography. Food Chem. Toxicol. 46, págs. 57-64.

[42] Landers, J. P. (ed.) (1997). Handbook of capillary electrophoresis, 2nd Ed., CRC Press. Boca Raton, FL, EEUU.

[43] Cruces Blanco, C. (1998). Electroforesis capilar, Servicio de Publicaciones, Universidad de Almería. Almería, España.

[44] Fernández-Gutiérrez, A.; Segura-Carretero, A. (eds.) (2005). Electroforesis capilar: aproximación según la técnica de separación. Editorial Universidad de Granada. Granada, España.

[45] Hernández-Borges, J.; Frías-García, S.; Cifuentes, A.; Rodríguez-Delgado, M. A. (2004). Pesticide analysis by capillary electrophoresis. J. Sep. Sci., 27, págs. 947-963.

[46] Juan-García, A.; Font, G.; Picó, Y. (2005). Determination of organic contaminants in food by capillary electrophoresis. J. Sep. Sci., 28, págs. 793-812.

[47] García-Campaña, A. M.; Gámiz-Gracia, L.; Lara, F. J.; del Olmo Iruela, M.; Cruces-Blanco, C. (2009). Applications of capillary electrophoresis to the determination of antibiotics in food and environmental samples. Anal. Bioanal. Chem., 395, págs. 967-986.

[48] González-Peñas, E.; Leache, C.; López de Cerain, A.; Lizarraga, E. (2006). Comparison between capillary electrophoresis and HPLC-FL for ochratoxin A quantification in wine. Food Chem., 97, págs. 349-354. 
[49] Almeda, S.; Arce, L.; Valcárcel, M. (2008). Combined use of supported liquid membrane and solid-phase extraction to enhance selectivity and sensitivity in capillary electrophoresis for the determination of ochratoxin A in wine. Electrophoresis, 29, págs. 1573-1581.

[50] Maragós, C. M. (2004). Detection of moniliformin in maize using capillary zone electrophoresis. Food Add. Contam., 21, págs. 803-810.

[51] Gökmen, V.; Acar, J.; Sarioglu, K. (2005). Liquid Chromatography method for the determination of patulin in apple juice using solid phase extraction. Anal. Chim. Acta, 543, págs. 64-69.

[52] Boonzaaijer, G.; Bobeldijk, I.; van Osenbruggen, W.A. (2005). Analysis of patulin in dutch food, an evaluation of a SPE based method. Food Control, 16 (7), págs. 587-591.

[53] Jian-ke L.; Ri-na W.; Qiu-hui H.; Jian-hua W. (2007). Solid-phase extraction and HPLC determination of patulin in apple juice concentrate. Food Control, 18, págs. 530-534.

[54] Hernández, M. A.; Valme, G. M.; Duran, E.; Guillen, D.; Barroso, C. G. (2006). Validation of two analytical methods for the determination of ochratoxin A by reversed-phased highperformance liquid chromatography coupled to fluorescence detection in musts and sweet wines from Andalusia. Anal. Chim. Acta, 566, págs. 117-121.

[55] Stecher, G.; Jarukamjorn, K.; Zaborski, P., Bakry, R., Huck, C. W., Bonn, G. K. (2007). Evaluation of extraction methods for the simultaneous analysis of simple and macrocyclic trichothecenes. Talanta, 73, págs. 251-257.

[56] Labuda, R.; Parich, A.; Vekiru, E.; Tancinova, D. (2005). Incidence of fumonisins, moniliformin and Fusarium species in poultry feed mixtures from Slovakia. Ann. Agric. Environ. Med., 12, págs. 81-86.

[57] Senyuva, H. Z.; Gilbert, J. (2010). Immunoaffinity column clean-up techniques in food analysis: A review. J. Chromatogr. B, 878, págs 115-132.

[58] Aresta, A.; Cioffi, N.; Palmisano, F.; Zamboni, C. G. (2003). Simultaneous determination of ochratoxin A and cyclopiazonic, mycophenolic, and tenuazonic acids in cornflakes by solidphase microextraction coupled to high-performance liquid chromatography. J. Agric. Food Chem., 51, págs. 5232-5237.

[59] Lagana, A.; Bacaloni, A.; Castellano, M.; Curini, R.; De Leva, I.; Faberi, A.; Materazzi, S. (2003). Sample preparation for determination of macrocylic lactone mycotoxins in fish tissue, based on on-line matrix solid-phase dispersion and solid-phase extraction clean-up followed by liquid chromatography/tandem mass spectrometry. J. AOAC Int., 86, págs. 729-736.

[60] Blesa, J.; Soriano, J. M.; Moltó, J. C.; Marin, R.; Mañes, J. (2003). Determination of aflatoxins in foods from local markets and supermarkets in Valencia, Spain. Food Addit Contam., 21, págs. 165-171.

[61] Tsao R.; Zhou T. (2000). Micellar Electrokinetic Capillary Electrophoresis for Rapid Analysis of Patulin in Apple Cider. J. Agric. Food Chem., 48, págs. 5231-5335.

[62] Selim, M. I.; Tsuei, M. H. (1993). Development and optimization of a supercritical fluid extraction method for the analysis of aflatoxin B1 in grain dust. Am. Ind. Hyg. Assoc. J., 54, págs. $135-141$. 
[63] Krska, R. (1998). Performance of modern sample preparation techniques in the analysis of Fusarium mycotoxins and its quality assurance. Food Addit Contam., 22, págs. 345-353.

[64] Pallaroni, L.; Von Holst, C. (2003). Comparison of alternative and concentional extraction techniques for the determination of zearalenone in corn. Anal. Bional. Chem., 376, págs. 908912.

[65] Royer, D.; Humpf, H. U.; Guy, P. A. (2004). Quantitative analysis of Fusarium mycotoxins in maize using accelerated solvent extraction before liquid chromatography/atmospheric pressure chemical ionization tandem mass spectrometry. Food Addit. Contam., 21, págs. 678-692.

[66] Juan, C.; González, L.; Soriano, J. M.; Moltó, J. C.; Mañes, J. (2005). Accelerated solvent extraction of ochratoxin A from rice samples. J. Agric. Food Chem., 53, págs. 9348-9351.

[67] Truckesess, M. W.; Tang, Y. F. (1999). Solid-phase extraction method for patulin in apple juice and unfiltered apple juice. J. AOAC Int., 82 (5), págs. 1109-1113.

[68] Rezaee, M.; Assadi, Y.; Milani Hosseini M.R.; Aghaee, E.; Ahmadi, F.; Berijani, S. (2006). "Determination of organic compounds in water using dispersive liquid-liquid microextraction", J. Chromatogr. A, 1116 (1-2), págs. 1-9.

[69] Herrera-Herrera, A. V.; Asensio-Ramos, M.; Hernández-Borges, J.; Rodríguez-Delgado, M.A. (2010). Dispersive liquid-liquid microextraction for determination of organic analytes. TRACTrends Anal. Chem., 29 (7), págs. 728-751.

[70] Hao C.; Ruiwen C.; Shengqing L. (2010). Low-density extraction solvent-based solvent terminated dispersive liquid-liquid microextraction combined with gas chromatography-tandem mass spectrometry for the determination of carbamate pesticides in water samples. J. Chromatogr. A, 1217, págs.1244-1248.

[71] Rezaee, M.; Yamini, Y.; Faraji, M. (2010). Review: Evolution of dispersive liquid-liquid microextraction method. J. Chromatogr. A, 1217, págs. 2342-2357.

[72] Bosch-Ojeda C.; Sánchez-Rojas, F. (2007). Separation and Preconcentration by Dispersive Liquid-Liquid Microextraction Procedure: A Review. Food Control 18, págs. 530-534.

[73] Campone L.; Piccinelli, A. L.; Rastrelli, L. (2010). Dispersive liquid-liquid microextraction combined with high-performance liquid chromatography-tandem mass spectrometry for the identification and the accurate quantification by isotope dilution assay of Ochratoxin A in wine samples. Anal. Bioanal Chem., 399, págs. 1279-1286.

[74] Arroyo-Manzanares, N.; Gámiz-Gracia, L.; García-Campaña, A.M. (2011). Determination of Ochartoxin A in wines by Capillary Liquid Charomatography with laser induced fluorescence detection using dispersive liquid-liquid microextraction. Food Chem. (en revisión). 